University of Tennessee Health Science Center UTHSC Digital Commons

\title{
Investigating the Regulation and Function of the NR4A Nuclear Receptors in Cancer
}

Jordan A. Beard

University of Tennessee Health Science Center

Follow this and additional works at: https://dc.uthsc.edu/dissertations

Part of the Medical Cell Biology Commons, and the Medical Molecular Biology Commons

\section{Recommended Citation}

Beard, Jordan A. (http://orcid.org/0000-0003-0281-1432), "Investigating the Regulation and Function of the NR4A Nuclear Receptors in Cancer" (2016). Theses and Dissertations (ETD). Paper 378.

http://dx.doi.org/10.21007/etd.cghs.2016.0391.

This Dissertation is brought to you for free and open access by the College of Graduate Health Sciences at UTHSC Digital Commons. It has been accepted for inclusion in Theses and Dissertations (ETD) by an authorized administrator of UTHSC Digital Commons. For more information, please contact jwelch30@uthsc.edu. 


\title{
Investigating the Regulation and Function of the NR4A Nuclear Receptors in Cancer
}

\author{
Abstract \\ The nuclear receptor (NR) superfamily represents a structurally-conserved group of ligand-regulated \\ transcription factors. These proteins have critical roles in various physiological and pathological \\ processes, including cancer, and have been targets of drug therapy. The orphan NR subfamily 4A (NR4A), \\ which includes the NR4A1 (Nur77), NR4A2 (Nurr1), and NR4A3 (Nor-1) genes, has been implicated in \\ adult solid tumors and has been characterized as pro-tumorigenic mediator of cell proliferation, \\ transformation, migration, and drug resistance. Alternatively, in leukemia, NR4A1 and NR4A3 have been \\ described as tumor suppressors in hematologic malignancies. Members of the NR4A family are \\ commonly overexpressed in cancer and this has been attributed to their regulation by other oncogenic \\ signaling pathways.
}

Despite the understanding of signaling cascades that lead to overexpression of the NR4A members, little is known about their regulation by microRNAs (miRNAs). miRNAs are small, non-coding, endogenous RNAs that are transcribed, processed, and used to direct cellular proteins that destabilize or block translation of target mRNA. In this study, we first sought to determine the miRNAs that are responsible for regulating NR4A2. Using a 3' UTR reporter assay, we identified miR-34 as a regulator of the NR4A2 through its $3^{\prime}$ UTR, which was confirmed using mutagenesis of the predicted binding region of the miR-34 seed region to its target site. We demonstrated that overexpression of exogenous or induction of endogenous miR-34 expression downstream of p53 activation by Nutlin-3a was associated with decreased endogenous NR4A2. Additionally, overexpression of NR4A2 was capable of suppressing the activation of p53 target genes, and was also able to attenuate the sensitivity of cells to the antiproliferative effect of Nutlin-3a.

We further explored the roles of the NR4A family in pediatric cancer, an area that has not been fully investigated. We first determined that the members of the NR4A family are overexpressed in rhabdomyosarcoma (RMS) cell lines compared to normal muscle cells. Knockdown of NR4A1 or NR4A2 led to a reduction in cell proliferation and transformation, while knockdown of NR4A2 could also affect cell migration. Using a microarray approach, we sought to investigate the transcriptome-level changes in response to NR4A knockdown, and determined that knockdown of NR4A2 led to a unique gene signature, while NR4A1 and NR4A3 knockdown had large overlaps in expression changes. These unique gene expression changes in response to NR4A2 knockdown could explain the unique effects that NR4A2 has on migration.

Overall, this study has discovered miR-34 as a novel regulator of NR4A2, and places NR4A2 in a potential feedback mechanism involving p53, miR-34, and NR4A2. This could indicate that NR4A2 mediates at least some of its pro-oncogenic effects through the inhibition of $p 53$, which is relieved by $p 53$ itself upon activation. Alternatively, NR4A2, is shown to have other roles in cancer progression, potentially through novel downstream target genes. These data may be used in understanding the effects of miR-34 replacement therapy, as this method of treatment is progressing through clinical trials, allowing us to understand the diverse regulator cascades being modulated.

\section{Document Type}

Dissertation

Degree Name

Doctor of Philosophy (PhD) 


\section{Program}

Biomedical Sciences

Research Advisor

Taosheng Chen, Ph.D.

\section{Keywords}

nuclear receptor, NR4A2, miRNA, miR-34, p53, rhabdomyosarcoma

\section{Subject Categories}

Medical Cell Biology | Medical Molecular Biology | Medical Sciences | Medicine and Health Sciences 
Investigating the Regulation and Function of the NR4A

Nuclear Receptors in Cancer

\author{
A Dissertation \\ Presented for \\ The Graduate Studies Council \\ The University of Tennessee \\ Health Science Center \\ In Partial Fulfillment \\ Of the Requirements for the Degree \\ Doctor of Philosophy \\ From The University of Tennessee
}

By

Jordan A. Beard

May 2016 
Chapter 2 (C) 2015 by Elsevier Inc.

All other material (C) 2016 by Jordan A. Beard.

All rights reserved. 


\section{DEDICATION}

This dissertation is dedicated to my loving wife, Carrie Beard, and my family for their love and support during my time in graduate school. I fully appreciate your continued encouragement throughout this journey. 


\section{ACKNOWLEDGEMENTS}

First and foremost, I want to thank my mentor, Dr. Taosheng Chen. Over the past six years, he has provided me with his overwhelming support and an outstanding laboratory environment in which I could learn and grow into the scientist that I am today. My success in the lab and in my scientific career can be attributed to his mentorship and his guidance in becoming an independent scientist.

I would like to thank my dissertation committee members, Drs. Leonard Lothstein, Leta Nutt, Edwards Park, and Gerard Zambetti for their guidance and constructive review of my research project. I am also grateful for your time spent on writing recommendations for travel awards and during my search for a postdoctoral fellowship.

I have had the privilege of working with a great group of scientists in the department of Chemical Biology and Therapeutics at St. Jude Children's Research Hospital (SJCRH). I have enjoyed the opportunity to gain feedback on my research while making great friends in the process. From the first day of my rotation, the members of the Chen research group have been the most welcoming group of people, and have taught me much and supported me greatly throughout my time in lab. I would like to give a special thank you to those members who went above and beyond to assist me, which includes Drs. Jing Wu, Ayesha Elias, Su Sien Ong, Yue-Ming Wang, Milu Cherian, and Jesse Bakke, Mrs. Alexa Farmer, Mrs. Jessica Hoyer, and Ms. Asli Goktug.

A very special thank you goes to my former Pediatric Oncology Education summer student, Justin Hills. Mr. Hills was of tremendous help during the summers of 2013 and 2014. Your adeptness at the bench and in your organizational skills made my first experience as a mentor incredibly great, and I learned much about how to teach novel ideas and unfamiliar techniques to someone with an eager mind. I am grateful for your time here, and your willingness to help with the project is of great value.

Other SJCRH faculty and staff have aided in my education and research. I would like to thank Dr. Mark Hatley and his lab for valuable discussions and for providing reagents that have aided in my research. SJCRH is a wonderful place to work, largely because of the great core facilities offered. I would like to thank Dr. Michael Wang and Mr. Granger Ridout of the Hartwell Center for their assistance with providing microarray processing and dataset analysis.

Last but not least, I want to thank Dr. Susan Nozell at the University of Alabama at Birmingham. Dr. Nozell was the first person to introduce me to hands-on biomedical research and I am grateful to have taken her Cell and Molecular Biology class. From discussions about cell signaling pathways in class, learning how to run PCR and Western blots in the lab, to demonstrating how to structure a grant application, I thank Dr. Nozell for instilling within me a passion for research. 


\begin{abstract}
The nuclear receptor (NR) superfamily represents a structurally-conserved group of ligand-regulated transcription factors. These proteins have critical roles in various physiological and pathological processes, including cancer, and have been targets of drug therapy. The orphan NR subfamily 4A (NR4A), which includes the NR4A1 (Nur77), $N R 4 A 2$ (Nurr1), and NR4A3 (Nor-1) genes has been implicated in adult solid tumors and have been characterized as pro-tumorigenic mediator of cell proliferation, transformation, migration, and drug resistance. Alternatively, in leukemia, NR4A1 and NR4A3 have been described as tumor suppressors in hematologic malignancies. Members of the NR4A family are commonly overexpressed in cancer and this has been attributed to their regulation by other oncogenic signaling pathways.
\end{abstract}

Despite the understanding of signaling cascades that lead to overexpression of the NR4A members, little is known about their regulation by microRNAs (miRNAs). miRNAs are small, non-coding, endogenous RNAs that are transcribed, processed, and used to direct cellular proteins that destabilize or block translation of target mRNA. In this study, we first sought to determine the miRNAs that are responsible for regulating $N R 4 A 2$. Using a 3' UTR reporter assay, we identified miR-34 as a regulator of the NR4A2 through its 3' UTR, which was confirmed using mutagenesis of the predicted binding region of the miR-34 seed region to its target site. We demonstrated that overexpression of exogenous or induction of endogenous miR-34 expression downstream of p53 activation by Nutlin-3a was associated with decreased endogenous NR4A2. Additionally, overexpression of $N R 4 A 2$ was capable of suppressing the activation of p53 target genes, and was also able to attenuate the sensitivity of cells to the anti-proliferative effect of Nutlin-3a.

We further explored the roles of the NR4A family in pediatric cancer, an area that has not been fully investigated. We first determined that the members of the NR4A family are overexpressed in rhabdomyosarcoma (RMS) cell lines compared to normal muscle cells. Knockdown of $N R 4 A 1$ or $N R 4 A 2$ led to a reduction in cell proliferation and transformation, while knockdown of $N R 4 A 2$ could also affect cell migration. Using a microarray approach, we sought to investigate the transcriptome-level changes in response to NR4A knockdown, and determined that knockdown of NR4A2 led to a unique gene signature, while $N R 4 A 1$ and $N R 4 A 3$ knockdown had large overlaps in expression changes. These unique gene expression changes in response to $N R 4 A 2$ knockdown could explain the unique effects that NR4A2 has on migration.

Overall, this study has discovered miR-34 as a novel regulator of NR4A2, and places NR4A2 in a potential feedback mechanism involving p53, miR-34, and NR4A2. This could indicate that NR4A2 mediates at least some of its pro-oncogenic effects through the inhibition of p53, which is relieved by p53 itself upon activation. Alternatively, NR4A2, is shown to have other roles in cancer, potentially through novel downstream target genes. These data may be used in understanding the effects of miR-34 
replacement therapy, as this method of treatment is progressing through clinical trials, allowing us to understand the diverse regulator cascades being modulated. 


\section{TABLE OF CONTENTS}

CHAPTER 1. INTRODUCTION ................................................................................

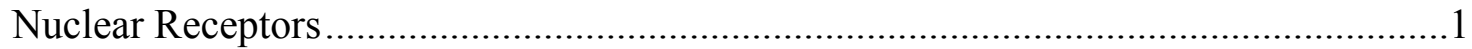

Discovery of the nuclear receptor superfamily .................................................... 1

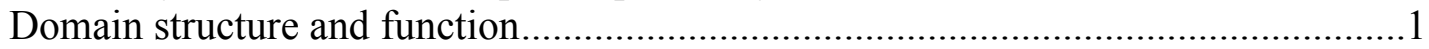

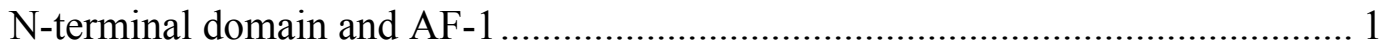

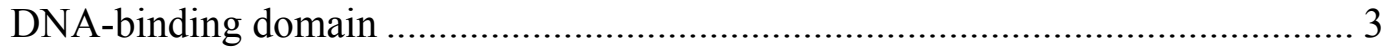

Ligand-binding domain and AF-2 .............................................................. 3

Nuclear receptors and cancer ..........................................................................

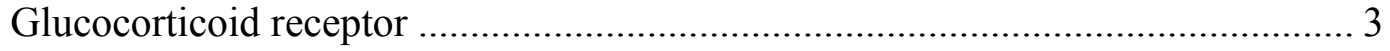

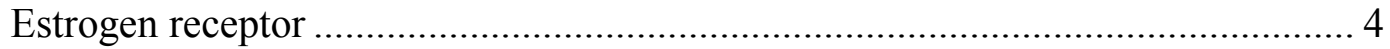

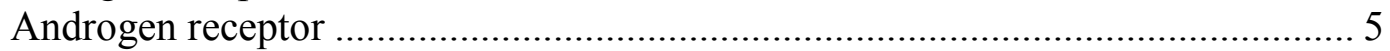

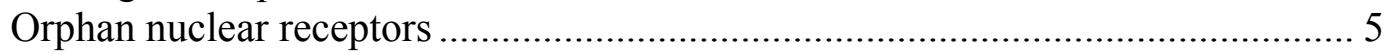

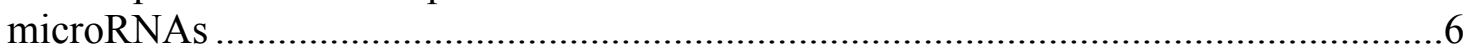

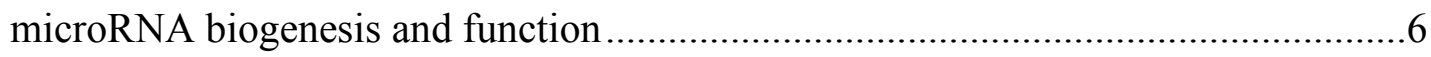

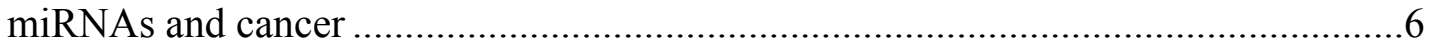

Tumor-suppressor-like miRNAs ............................................................ 9

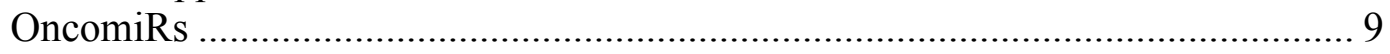

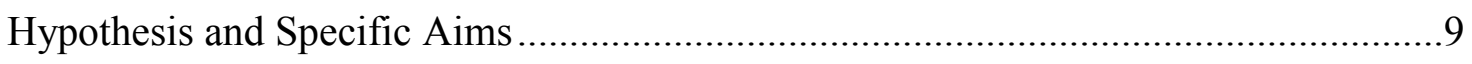

\section{CHAPTER 2. THE INTERPLAY OF NR4A RECEPTORS AND THE} ONCOGENE-TUMOR SUPPRESSOR NETWORKS IN CANCER........................11

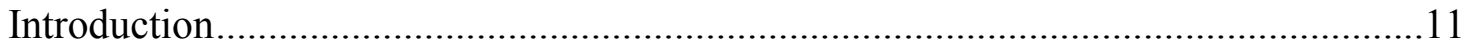

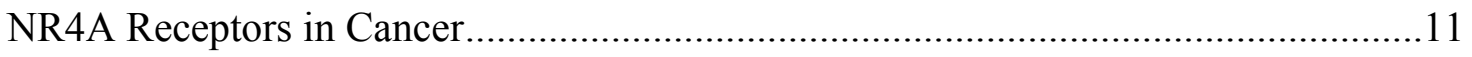

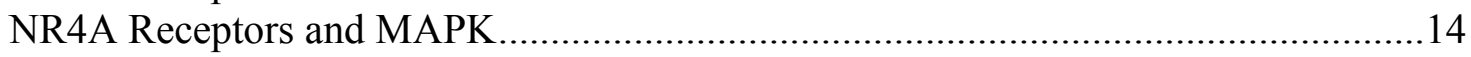

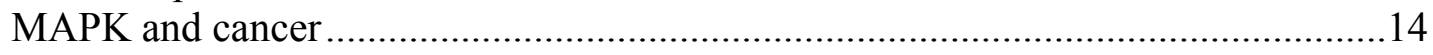

Regulation of NR4A receptors by MAPK .......................................................... 15

NR4A Receptors, PI3K-AKT, and mTOR in Proliferation and Survival ....................16

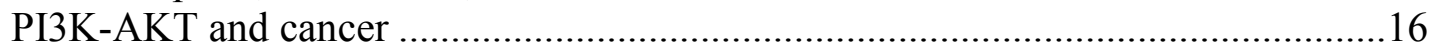

The interplay of NR4A receptors and PI3K-AKT ................................................. 16

The interplay of NR4A receptors and mTOR ................................................... 17

NR4A Receptors, Hypoxia, and Angiogenesis ................................................. 18

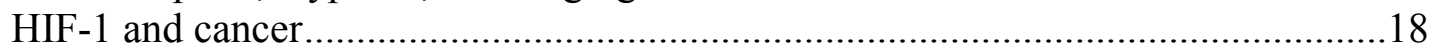

The interplay of NR4A receptors and HIF-1 ...................................................19

NR4A receptors as mediators of VEGF-induced angiogenesis ..............................20

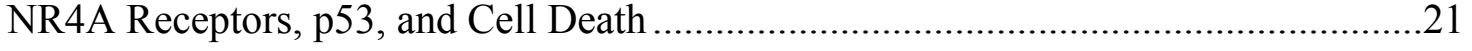

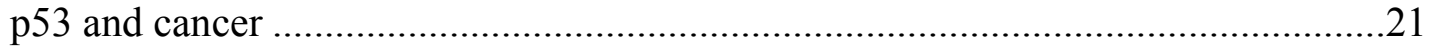

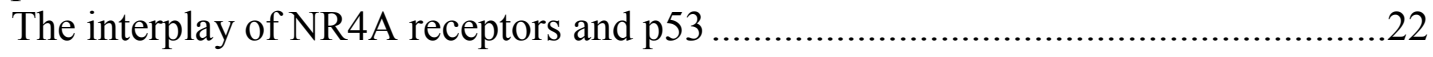

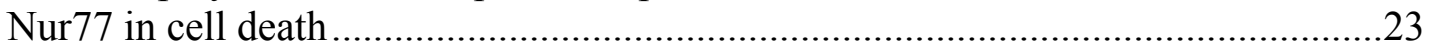

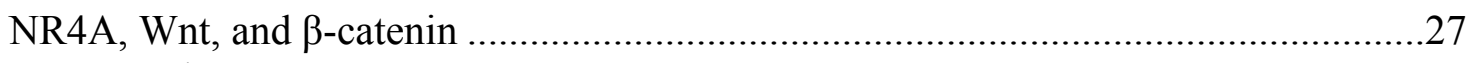

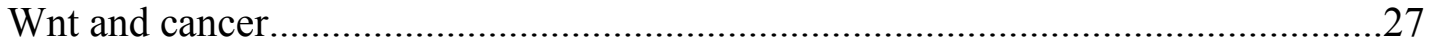

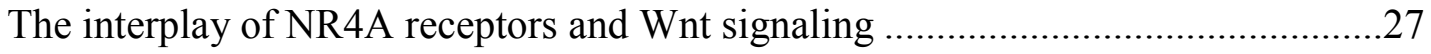

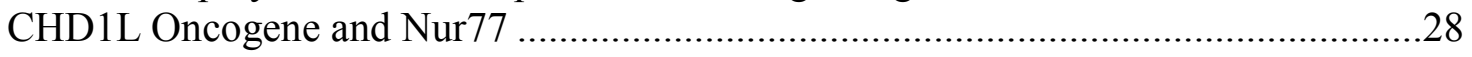

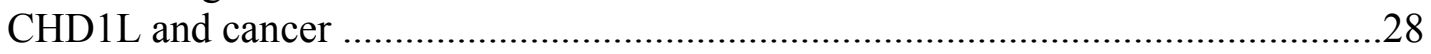




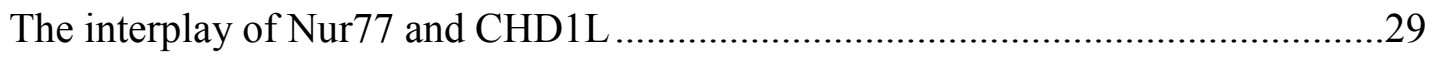

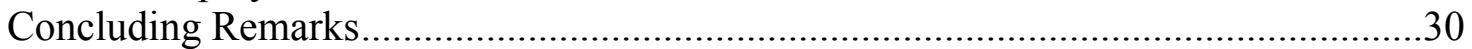

\section{CHAPTER 3. THE ORPHAN NUCLEAR RECEPTOR NR4A2 IS PART OF A} P53-MICRORNA-34 NETWORK......................................................................33

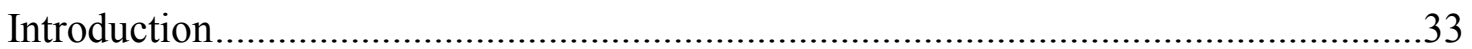

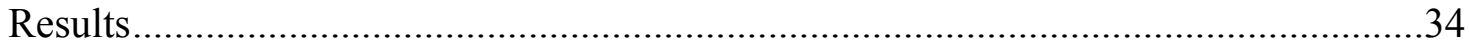

Identification of miRNAs directly targeting the $3^{\prime}$ UTR of NR4A2 ……...................34

miR-34 regulates endogenous $N R 4 A 2$ levels ..........................................................37

p53 activation suppresses endogenous $N R 4 A 2$ levels...............................................39

Overexpression of $N R 4 A 2$ suppresses p53 activation...............................................41

Knockdown of NR4A2 enhances p53 activation .....................................................41

Overexpression of $N R 4 A 2$ attenuates Nutlin-3a sensitivity .......................................4

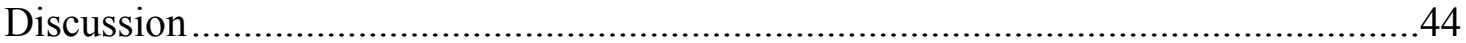

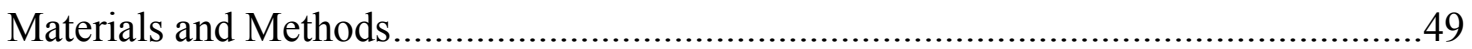

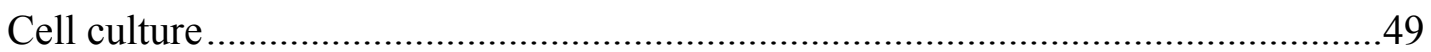

3' UTR reporter plasmid and microRNA screen...................................................50

miRNA target prediction and mutagenesis ........................................................50

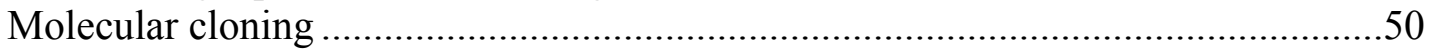

miRNA mimics, RNAi, and chemical treatments ....................................................50

RNA extraction and quantitative real-time PCR ..................................................51

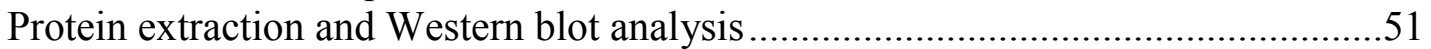

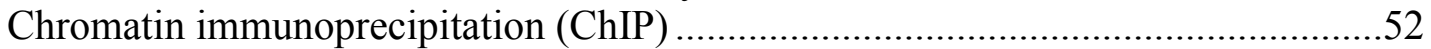

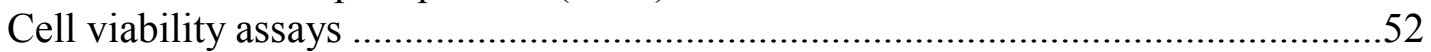

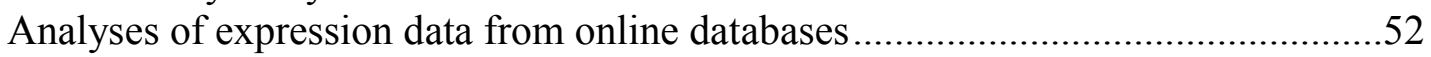

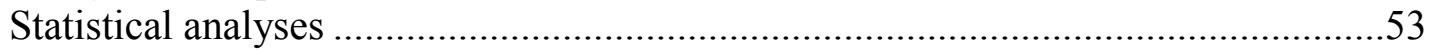

\section{CHAPTER 4. INVESTIGATING THE EXPRESSION AND FUNCTION OF} NR4A NUCLEAR RECEPTORS IN RHABDOMYOSARCOMA ..............................54

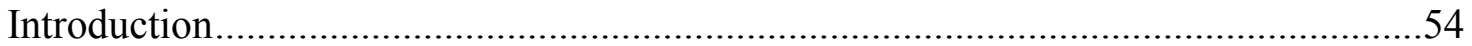

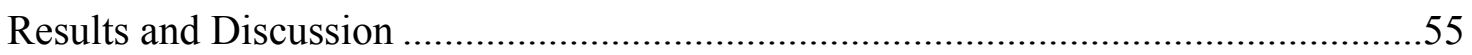

NR4A nuclear receptors are overexpressed in RMS ………………………….......55

Knockdown of NR4A1 and NR4A2 decreases cell proliferation.................................55

Knockdown of NR4A2 decreases cell migration.....................................................58

Knockdown of NR4A1 and NR4A2 decreases colony formation ..............................58

$N R 4 A 2$ knockdown leads to an altered transcriptional profile.....................................62

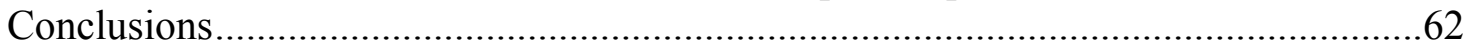

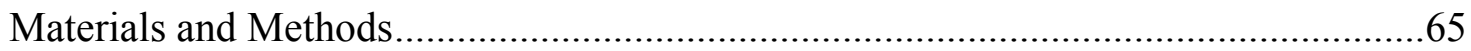

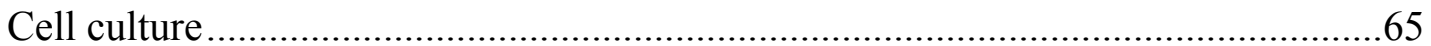

Transfection of siRNA, RNA extraction, and quantitative real-time PCR .................65

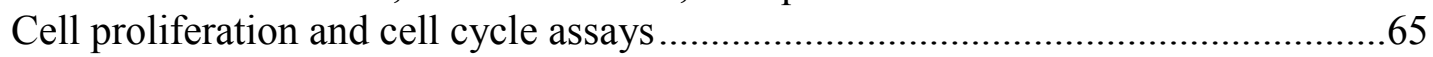

Cell migration assays ......................................................................................66

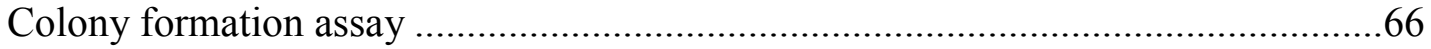

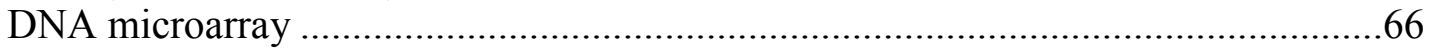




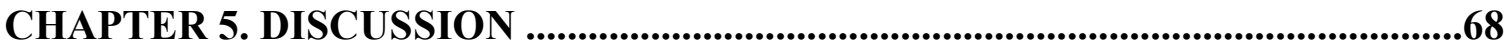

NR4A Regulation by miRNAs in Cancer...............................................................68

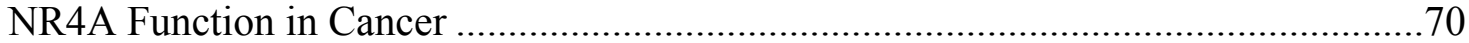

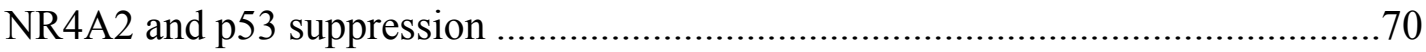

NR4A receptors in rhabdomyosarcoma..............................................................

LIST OF REFERENCES .................................................................................................72

APPENDIX. SUPPLEMENTAL DATA FOR CHAPTER 3........................................88

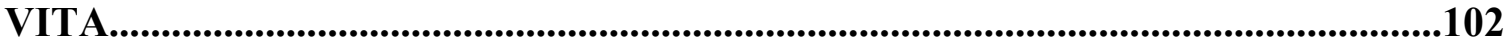




\section{LIST OF TABLES}

Table 2-1. Summary of gene expression changes after treatment with NR4A agonists or antagonists.

Table 3-1. MicroRNAs that putatively regulate NR4A2 through its $3^{\prime}$ UTR................36

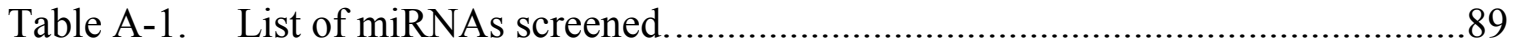

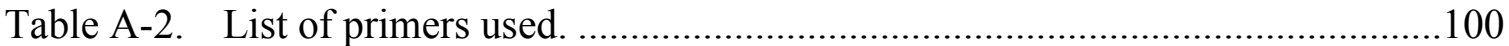




\section{LIST OF FIGURES}

Figure 1-1. The nuclear receptor signaling pathway. ..............................................2

Figure 1-2. The microRNA biogenesis pathway....................................................

Figure 1-3. Dysregulation of miRNAs can contribute to cancer. .................................

Figure 2-1. Nur77 mediates cell death or survival through localization-dependent and -independent mechanisms........................................................ 13

Figure 2-2. The NR4A family and key signaling pathways regulate each other............31

Figure 3-1. Screening for miRNAs that directly target the $3^{\prime}$ UTR of NR4A2 ...............35

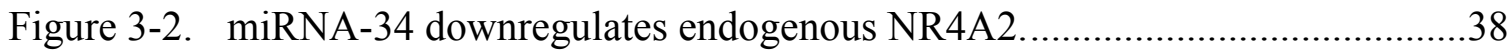

Figure 3-3. Nutlin-3a activation of p53 decreases endogenous NR4A2 .....................40

Figure 3-4. NR4A2 overexpression suppresses p53 activation.................................42

Figure 3-5. Knockdown of NR4A2 enhances p53 activation.....................................43

Figure 3-6. Overexpression of $N R 4 A 2$ attenuates Nutlin-3a sensitivity.......................45

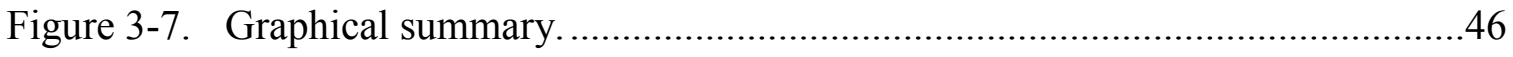

Figure 4-1. NR4A nuclear receptors are overexpressed in rhabdomyosarcoma cell

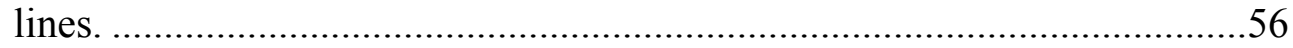

Figure 4-2. Knockdown of NR4A1 and NR4A2 decreases cell proliferation..................57

Figure 4-3. Knockdown of NR4A2 decreases cell migration. ....................................59

Figure 4-4. Knockdown of $N R 4 A 1$ and $N R 4 A 2$ decreases colony formation.................61

Figure 4-5. Gene expression following NR4A knockdown. .....................................63

Figure A-1. Correlation of miR-34a and NR4A2 expression in rectum adenocarcinoma patients. ............................................................... 91

Figure A-2. miR-34a correlation matrix in rectum adenocarcinoma patients................93

Figure A-3. Overexpression of miR-34 increases p53 protein and acetylation levels. ....95

Figure A-4. Overexpression of miR-34 decreases NR4A2 in RKO colorectal cancer cells. 
Figure A-5. Nutlin-3a enhances p53 and p21 protein levels in RKO and SW48 colorectal cancer cell lines.

Figure A-6. p53 activation by Nutlin-3a decreases NR4A2 in RKO and SW48 cell lines.

Figure A-7. Overexpression of NR4A2 does not affect binding of p53 to target gene promoters.

Figure A-8. Validation of an anti-NR4A2 antibody 101 


\title{
CHAPTER 1. INTRODUCTION
}

\author{
Nuclear Receptors
}

\section{Discovery of the nuclear receptor superfamily}

Three decades ago, the glucocorticoid (GR) and estrogen (ER) receptors were the first described nuclear receptors (NRs) [1-4], and since then much has been discovered in regard to this group of key transcriptional regulators in physiological and pathological events. Following characterization of GR and ER, additional proteins which shared conserved domains began to be discovered [5], and now the human NR superfamily consists of 48 genes that encode a highly homologous set of transcription factors. This superfamily of proteins shares similar structural organization, composed of modular domains $[6,7]$ that include (listed from $\mathrm{N}$ - to C-terminal) an N-terminal domain which harbors an activation function-1 (AF-1) region, a DNA-binding domain (DBD), a flexible hinge region, and a $\mathrm{C}$-terminal ligand-binding domain (LBD) that contains an AF-2 region.

These mostly ligand-regulated transcription factors control target gene expression through mechanisms typically involving ligand binding, dimerization, nuclear localization, and recruitment and association with coregulators at sites in gene promoters (Figure 1-1). Upon initial cloning of the various NR members, those lacking a defined ligand were termed 'orphan' receptors - some of which have remained 'orphans,' while others have been 'adopted' following identification of their cognate ligand. Those with known ligands typically are activated by steroid or thyroid hormones, vitamins, fatty acids, bile acids, sterols, or xenobiotics [8]. Several key members of the NR family have been utilized as drug targets $[9,10]$, and it was reported that $13 \%$ of FDA-approved drugs were NR modulators, representing the second-leading class of drug targets at that time [11]. Additionally, other NRs, such as PXR and CAR, have been studied for their roles in regulating transcriptional target genes that are involved in drug metabolism, having implications in drug-drug interactions and drug toxicity $[12,13]$.

\section{Domain structure and function}

\section{N-terminal domain and AF-1}

The N-terminal domain of NRs is divergent among the different NR subfamilies and isoforms, with large variability in the size of this domain. Encoded in the N-terminal domain is the AF-1 region, which can function independently of ligand binding [14]. This region is also important in post-translational regulation of NR function through phosphorylation or protein-protein interactions [15-18]. These regulatory mechanisms can serve to affect the protein levels or transcriptional activity of the NR. 


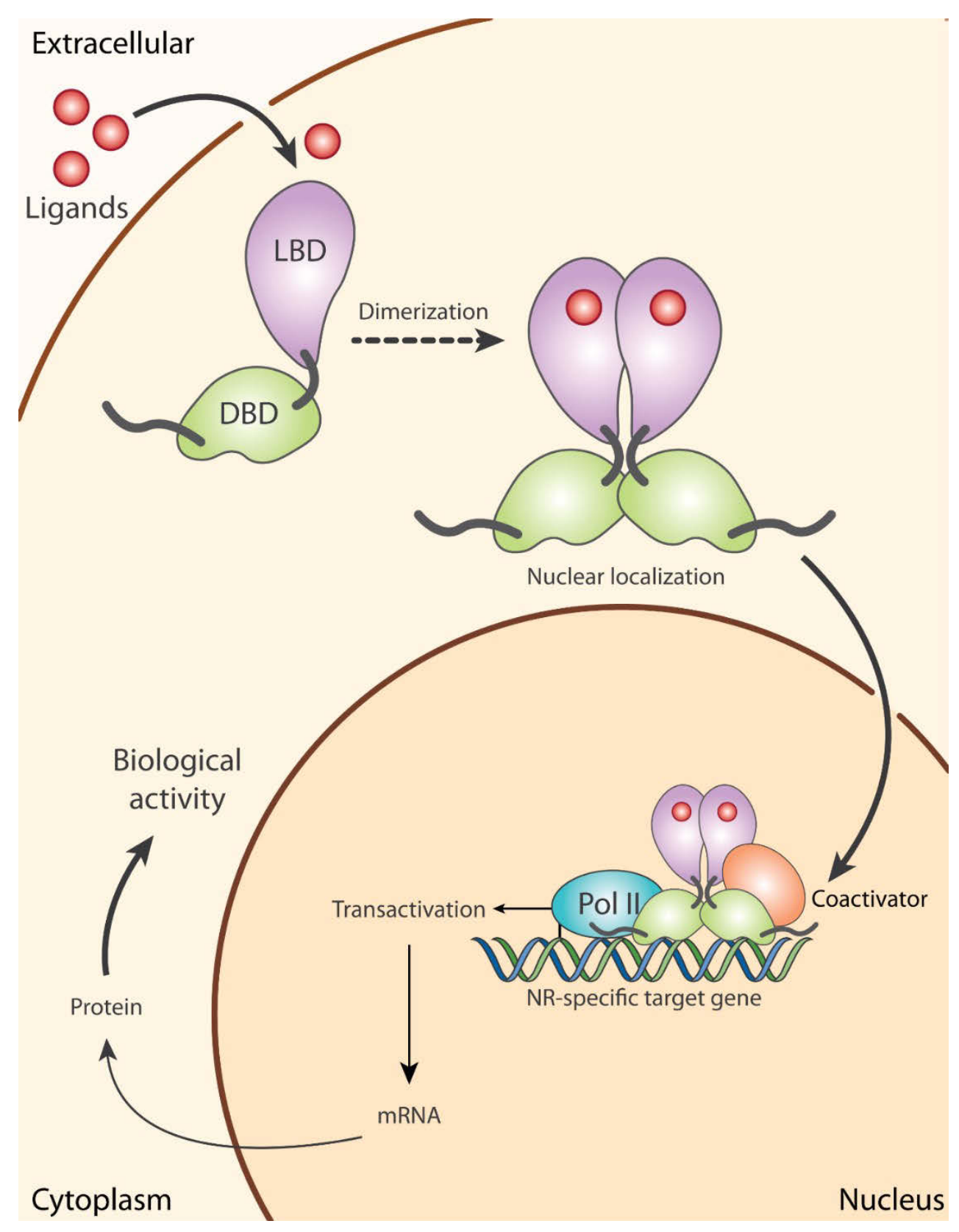

\section{Figure 1-1. The nuclear receptor signaling pathway.}

The nuclear receptor (NR) signaling cascade begins with an endogenous or synthetic ligand for a specific NR enters the cell and binds to the ligand-binding domain (LBD) of the NR. This triggers a dimerization of either homo- or hetero- NR binding partners. This binding causes an exposure of a nuclear localization signal, at which time the dimer is translocated to the nucleus where it recruits coactivators and the transcriptional machinery. This complex directs the transcription of NR target genes through its DNAbinding domain (DBD) depending on specific recognition elements in the DNA. The resultant gene is transcribed into protein and subsequently mediates the biological response of NR activation. 


\section{DNA-binding domain}

The DBD is the most conserved region among the members of the NR superfamily. This region contains two zinc finger motifs, which direct the DNA-binding activities of each NR to double stranded DNA at receptor-specific response elements [19]. These DNA response elements have specific sequences to confer the NR-specific binding activities for the regulation of corresponding target genes. The DBD also contains a nuclear localization signal (NLS) which, when exposed, directs NR complexes to the nucleus upon ligand binding [20].

\section{Ligand-binding domain and AF-2}

The LBD is the second most conserved region among NRs, and is the domain that makes NRs unique from other transcription factors. The LBD is responsible for binding endogenous or synthetic ligands to activate downstream NR signaling. This domain also contains the AF-2 region, which directs ligand-dependent recruitment of coregulators [14]. The LBD encodes the major interface for NR dimerization upon ligand binding [21]. Using sequence-based and crystallographic studies, it was determined that NRs share common folding in the LBD region [22], which consists of a series of $\alpha$-helices, referred to as $\mathrm{H} 1-\mathrm{H} 12$. These helices form the pocket in which the ligand binds. Binding of ligand causes a shift of the helices with $\mathrm{H} 12$ closing the cavity for a more stable holoconformation [23]. H12 encodes part of the AF-2 which, when closed in the holo-form, presents a hydrophobic cleft for the coactivator recruitment.

\section{Nuclear receptors and cancer}

In addition to NRs having important roles in normal physiological contexts, NR dysregulation can occur and lead to various disease states including cancer. Examples of this include several hormone-dependent cancers, such as breast and prostate cancer, where NRs play a critical role in the development, progression, and resistance of these malignancies to therapy. As a result, these NRs have been targeted by small molecule modulators and hormone therapy as a way to treat disease.

\section{Glucocorticoid receptor}

The glucocorticoid receptor (GR) serves as the receptor for natural (cortisol) and synthetic (dexamethasone, prednisone/prednisolone, and budesonide) glucocorticoids (GCs). As the physiological function of GCs are to minimize inflammation, these steroid molecules were first used therapeutically for the treatment of rheumatoid arthritis [24]. Synthetic GCs are commonly prescribed for various diseases with inflammatory conditions, including asthma and dermatitis [25]. GCs have also been used in the treatment of lymphoid malignancies, including acute lymphoblastic leukemia (ALL) [26]. 
The therapeutic response to GCs in ALL can be attributed primarily to the transcriptional activity of GR and is dependent on GC binding to GR [27]. Binding of GC to GR leads to cytoplasmic-to-nuclear translocation of GR, where it can then bind to target gene promoters and cause transactivation or repression of gene expression. Some of the GC-mediated effects include inhibition of cell proliferation and induction of apoptosis. Genes induced by GCs include Bim, Bid, and Bad, which promote apoptosis, while genes involved in inhibition of apoptosis (Bcl-2, Mcl-1, and Bcl-xL) are suppressed by GCs [27], leading to a GC-mediated apoptosis during therapy. Resistance to GCs can occur through various methods, including low GR expression in cells [28], mutations in GR $[29,30]$, or other alterations that affect GR directly such as overexpression of CASP1 [31].

\section{Estrogen receptor}

The estrogen receptor alpha $(\mathrm{ER} \alpha)$ and beta $(\mathrm{ER} \beta)$ are receptors for endogenous estrogens, which includes estrone (E1), 17 $\beta$-estradiol (E2), and estriol (E3). Estrogen is important in normal physiology, and is normally secreted by the ovaries in premenopausal women. High levels of estrogen have been associated with cancer in various tissues of both women and men, although the understanding of estrogens in cancer progression is understood predominantly from studies in breast and endometrial cancers [32]. The ability for estrogen to promote cancer development and progression is mediated mostly through ER $\alpha$-mediated transcriptional activation of target genes which include c-Myc [33] and cyclin D1 [34] to promote cell proliferation, and Bcl-2 [35] to block the apoptotic response and promote cell survival. As a normal physiological response of promoting new blood vessel formation (angiogenesis) in the uterus, estrogen can also induce genes responsible for this process in breast cancer cells [36], which is a critical component of tumor progression, allowing new blood flow to developing tumor. Although ER expression appears important for primary tumor formation, the loss of ER expression portends a more aggressive, invasive, and deadly disease [37] with a high propensity to metastasize, at which time targeting ER is no longer feasible.

Targeting of ER $\alpha$ with small molecule modulators has been a focus, and somewhat effective, strategy of treating hormone-dependent cancers. Tamoxifen, developed as the first selective estrogen receptor modulator (SERM), is able to reduce breast cancer recurrence and mortality, and is used as the front line drug for ER-positive breast cancers. SERMs are small molecules that are able to mimic estrogen and bind to the ER LBD, but rather act as an antagonist of ER function by blocking endogenous estrogen signaling through this axis [38]. Development of resistance is a major problem of tamoxifen and other therapies for breast cancer patients. Cytochrome P450 enzyme CYP2D6 is the primary metabolizer of tamoxifen into its active metabolite and, as a means of resistance, CYP2D6 polymorphisms leads to lower clinical efficacy [39]. 


\section{Androgen receptor}

The androgen receptor (AR) serves as the ligand for endogenous testosterone and its more active metabolite form, $5 \alpha$-dihydrotestosterone. These androgens are formed in the testes by Leydig cells, and serve to regulate gene expression that primarily directs male sexual development. Androgens and AR also stimulate proliferative and survival

pathways in prostate cells, and the dysregulation of these actions leads to development of prostate cancer [40]. A common test to indicate the need for patient surveillance or biopsy is measurement of serum levels of prostate-specific antigen (PSA), a wellcharacterized target gene of AR [41], which indicates increased androgen signaling. Studies have shown that 159 different mutations in AR itself can predispose men to develop prostate cancer [42].

Classically, removal of the testes was found to cause prostate cancer regression through the deprivation of androgen stimulation. Synthetic, non-steroidal antiandrogens have been developed that are capable of blocking androgen signaling, and these include flutamide, bicalutamide, and nilutamide [40]. Although structural studies have not confirmed their mechanism of action, it is believed that these antiandrogens bind to the LBD of AR, competing out the endogenous androgen binding. Resistance to androgen deprivation and blockade can develop, at which point the disease becomes deadly and difficult to treat, and is referred to as castration-resistant prostate cancer (CRPC) [43]. Several mechanisms of CRPC have been described, including increased AR expression and mutations in AR which cause it to either become activated by other endogenous steroids as a means to bypass the need for androgens or insensitive to the actions of antiandrogens.

\section{Orphan nuclear receptors}

Those NRs which have no identified endogenous ligands are classified as orphan NRs. This subset of receptors affect a multitude of physiological responses. Due to the absence of identified ligands, studies suggest that these NRs can act independently of ligand through constitutive activation, influenced through post-translational modifications or protein-protein interactions. Much like their ligand-associated family members, the orphan NRs can positively or negatively regulate gene expression through their DBD region.

Additionally, the actions of orphan NRs have been linked to cancer development and progression [44], which includes the known functions of one orphan NR subfamily, the NR4A receptors. The NR4A family, which includes NR4A1 (Nur77), NR4A2 (Nurr1), and NR4A3 (Nor-1), has been demonstrated to primarily have oncogenic roles in many adult solid tumors as a result of oncogene and tumor suppressor signaling in cancer [45]. NR4A1 and NR4A3 have also been shown to be downregulated and act as tumor suppressors in leukemia, with deletion of these NRs leading to rapid acute myeloid leukemia (AML) in mice [46]. Research primarily led by Safe and colleagues has targeted the NR4A receptors, primarily NR4A1, through the development of small molecule methyl-substituted diindolylmethanes (C-DIM) as inhibitors of 
NR4Amoncogenic activity [47-49]. Alternatively, some C-DIMs and another compound, cytosporone B, can activate proapoptotic functions of NR4A1 [49-55]. Another route of NR4A targeting has included altering the subcellular localization of NR4A1 to promote mitochondrial-associated apoptosis [56] or through the targeting of NR4A1 interactions with other proteins [57-59].

\section{microRNAs}

\section{microRNA biogenesis and function}

microRNAs (miRNAs) are small, endogenous, non-coding RNAs of approximately 22 nucleotides in length. Genes encoding miRNAs are transcribed from DNA by RNA polymerase II (Pol II) [60]. miRNA genes can be encoded in introns or exons of non-coding or protein-coding regions, and can exist alone or as a polycistronic transcript of multiple miRNAs. Transcription by Pol II forms a primary miRNA (primiRNA), which is then processed by the RNase Drosha to form a precursor miRNA (premiRNA) [61]. The pre-miRNA is exported from the nucleus by Exportin 5 into the cytoplasm [62]. Further processing occurs in the cytoplasm by the RNase Dicer, cleaving it into a small RNA duplex [63]. This short RNA duplex is loaded into a pre-RNAiinduced silencing complex (RISC), which contains an Argonaute (AGO) protein [64]. The pre-RISC is responsible for unwinding the miRNA duplex, retaining only the active strand.

Upon formation of the mature miRNA-containing RISC, this active complex is then directed primarily to $3^{\prime}$ untranslated regions (UTR) in target mRNAs, acting as a guide to direct gene or protein suppression [65]. The miRNA recognizes its targets based on nucleotide complementarity within the 3' UTR, and partial pairing of the mature miRNA with the $3^{\prime}$ UTR can be sufficient for target recognition [66]. The critical nucleotides for miRNA-mediated suppression are in positions $2-7$ at the $5^{\prime}$ end of the miRNA, which is referred to as the seed region of the miRNA [67]. The RISC then directs mRNA degradation, destabilization, or translational repression of target mRNAs [65], resulting in miRNA-mediated suppression of protein expression (Figure 1-2) and ultimately reduced protein function and biological activity.

\section{miRNAs and cancer}

Dysregulation of miRNAs has been attributed to the development and progression of cancer [68]. This is accomplished depending on the particular genes that a miRNA is responsible for targeting, with an alteration in the expression of the miRNA causing a resultant change in its target genes. Much like protein-coding genes that are involved in cancer, miRNAs can be classified as either oncomiRs or tumor-suppressor-like miRNAs (Figure 1-3). 


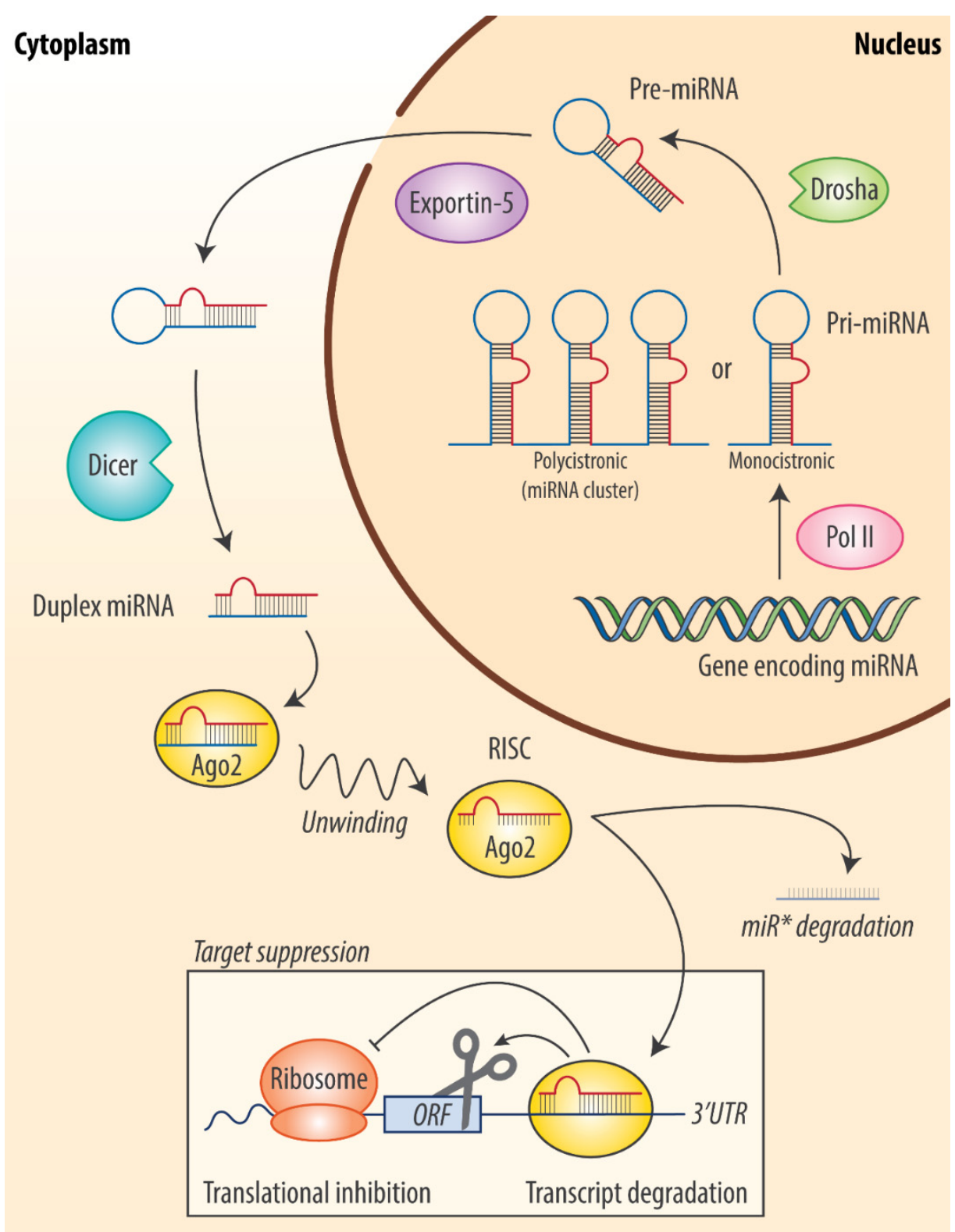

Figure 1-2. The microRNA biogenesis pathway.

MicroRNAs (miRNAs) are first transcribed from genes encoding each respective miRNA by RNA polymerase II (Pol II). The resulting transcript is referred to as pri-miRNA, and can exist as a single miRNA (monocistronic) or as a miRNA cluster (polycistronic). The pri-miRNA is then processed by the RNAase Drosha to form the pre-miRNA form. The pre-miRNA is then exported from the nucleus into the cytosol by Exportin-5, where it becomes further processed by the RNase Dicer. The duplex miRNA now incorporates into an Argonaute (Ago2)-containing RNAi-induced silencing complex, where the duplex unwinds and the unstable star-strand ( $\mathrm{miR}^{*}$ ) becomes degraded. Using the loaded active miRNA strand as a guide, the RISC binds to the 3' UTR of miRNA target genes, leading to mRNA transcript degradation or inhibition of ribosomal translation of mRNA into protein. 
Tumor suppressor-like miRNA

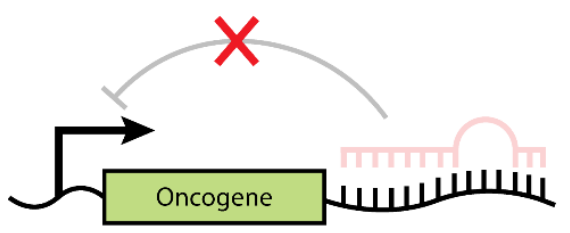

Loss of miRNA expression Increased oncogene expression

\section{OncomiR}

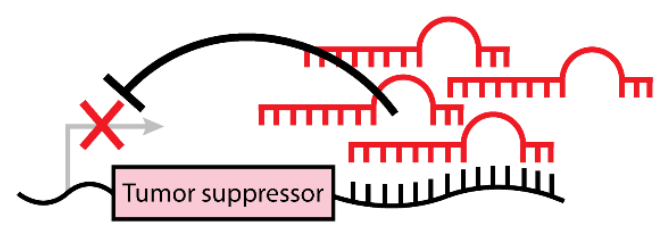

Overexpression of miRNA

Decreased tumor suppressor expression

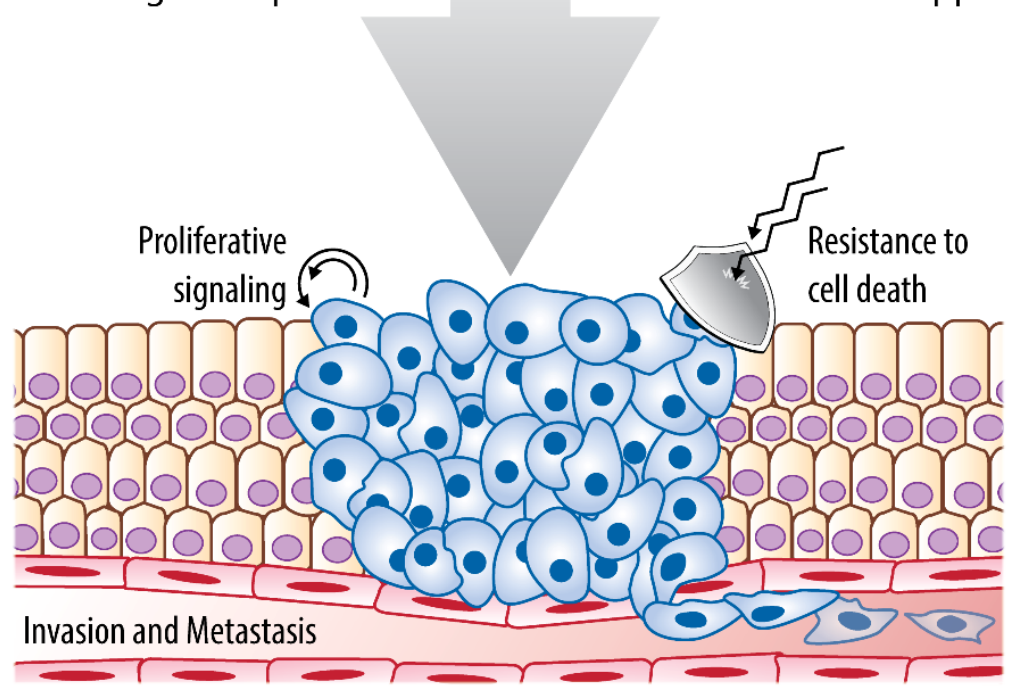

Figure 1-3. Dysregulation of miRNAs can contribute to cancer.

MiRNAs are able to act as tumor suppressors or oncogenes (referred to as oncomiRs) depending on the target genes that they suppress. Tumor-suppressor like miRNAs are described as targeting a gene that normally acts as an oncogene, but when this miRNA has reduced or absent expression, the oncogene is no longer suppressed and can increase in expression to promote cancer phenotypes. An oncomiR is a miRNA that targets a tumor suppressor, and the aberrant overexpression of this miRNA leads to decreased tumor suppressor expression and function, resulting in unchecked tumorigenesis. Cancerrelated miRNAs have been shown to contribute to multiple steps of cancer development, progression and metastasis, and therapeutic resistance. 


\section{Tumor-suppressor-like miRNAs}

Tumor-suppressor-like miRNAs are those miRNAs that typically are responsible for regulating genes that promote cell proliferation, survival, or other tumor-promoting processes. As are tumor suppressor proteins, the expression of tumor-suppressor-like miRNAs are decreased or lost due to factors such as loss in transcription factor regulation, epigenetic silencing, or genomic deletion [69]. This loss in miRNA expression allows the resulting target gene to become increased in expression, contributing to factors that promote cancer at multiple steps of oncogenesis.

One example of a tumor-suppressor-like miRNA is the miR-34 family. This family of miRNAs consists of three isoforms (miR-34a/b/c) that share a common seed region. The miR-34a family is transcriptionally regulated by the tumor suppressor protein p53 [70, 71], and has been attributed to partially carrying out tumor suppressive functions of the p53 response. As such, loss of p53 activity through deletion or mutation could be attributed to a decrease in miR-34 expression. Additionally, miR-34 expression can be lost through epigenetic silencing [72] or through deletions, as in the case of neuroblastoma $[73,74]$. Genes that are regulated by miR-34 include those involved in cell cycle progression, antiapoptotic and survival responses, and migration and metastasis [75].

\section{OncomiRs}

Alternatively, miRNAs can act similar to oncogenes. These miRNAs are termed oncomiRs, and are responsible for regulating genes that normally have tumor suppressive function. OncomiRs become increased in expression, typically by increased transcription factor signaling at the transcriptional level [68]. One example of an oncomiR includes miR-21, which was the first described oncomiR [76]. miR-21 regulates genes such as PTEN [77] and other tumor suppressor genes to elicit it's cancer-promoting effects, and it is found to be overexpressed in many tumor types.

\section{Hypothesis and Specific Aims}

Research by other groups suggests that overexpression of NR4A1 and NR4A2 could promote cell proliferation, migration, transformation, and survival in cancer. Understanding how members of the NR4A subfamily are regulated and determining their roles in cancer is the first step in understanding their biological relevance and usefulness as therapeutic targets. Although much is known about the cell signaling events that regulate NR4A expression, very little is known regarding the contributions of miRNAs in regulating this NR subfamily. Additionally, the function of NR4A NRs in pediatric malignancies has not been investigated.

We hypothesize that due to the previously described overexpression of NR4A

family members in solid tumors, particularly NR4A1 and NR4A2, that regulation of the NR4A members by tumor-suppressor-like miRNAs is a contributing factor to their 
overexpression, and that in turn, NR4A family members contribute to cancer phenotypes through their control of gene expression. To test this hypothesis, we carried out the following specific aims:

1. Investigate the regulation of NR4A2 by miRNAs and characterize the relevance of this regulatory mechanism in cancer.

2. Determine the roles of the NR4A receptors in caner and examine the gene expression changes as a result of NR4A modulation. 


\section{CHAPTER 2. THE INTERPLAY OF NR4A RECEPTORS AND THE ONCOGENE-TUMOR SUPPRESSOR NETWORKS IN CANCER*}

\section{Introduction}

The human nuclear receptor (NR) family is a group of structurally related transcription factors that regulate specific gene expression in a ligand-dependent manner. This superfamily of receptors constitutes an important group of drug targets that are useful in identifying compounds that affect a wide range of physiological and pathological events [9]. NRs share a common structural arrangement that consists of an N-terminal domain containing an activation function-1 (AF-1) region, a DNA-binding domain, a hinge region, and a $\mathrm{C}$-terminal ligand-binding domain (LBD) that can also encode an AF-2 domain. NR subfamily 4 group A (NR4A) is composed of three members: Nur77 (NR4A1, also known as nerve growth factor IB or NGFIB), Nurr1 (NR4A2), and Nor-1 (NR4A3).

Members of the NR4A subgroup respond to various stimuli, and their expression can be induced by mitogens, stress, and apoptotic signals, implicating their roles in multiple biological processes $[78,79]$. The NR4A receptors are classified as orphan receptors, having no known physiological ligands, and do not contain a typical ligandbinding domain structure common to other NRs [78, 80-82] although recent evidence suggests that unsaturated fatty acid metabolites could serve as the missing ligand for Nur77 [83]. Typical NRs have a ligand-binding domain containing a hydrophobic cleft for ligand- and coactivator-binding, but structural studies show that the NR4A subgroup contains an atypical ligand-binding groove that is hindered by bulky side groups of hydrophobic residues. Thus, the NR4A receptors are believed to be regulated in a ligandindependent manner, and a growing amount of literature supports the notion that these receptors are regulated largely by post-translational modifications and protein-protein interactions and that their expression and localization within the cell influences their cellular functions.

\section{NR4A Receptors in Cancer}

The NR4A receptors promote or suppress tumors depending on specific cellular context. For example, Nur77 is overexpressed in cancer cell and tissue samples of multiple origins, causing increased proliferation and survival in these cells and tissues [84] at least partly via upregulation of several target genes, including cyclin D2 [16], E2F1 [85], survivin [47], and thioredoxin domain-containing 5 (TXNDC5) [48], which

\footnotetext{
* Reprinted with permission from Elsevier. Beard, J.A., A. Tenga, and T. Chen, The interplay of NR $4 A$ receptors and the oncogene-tumor suppressor networks in cancer. Cell Signal, 2015. 27(2): p. 257-66. doi: http://dx.doi.org/10.1016/j.cellsig.2014.11.009
} 
are mediators of cell cycle progression, apoptotic inhibition and reactive oxygen species (ROS) regulation (Figure 2-1). In addition, loss-of-function studies of Nur77 have demonstrated its importance in cell proliferation and survival [86], with the consensus being that Nur77 knockdown reduces cellular growth rate and angiogenesis and induces intrinsic and extrinsic apoptotic pathways. It is important to note that many loss-offunction studies are performed on non-stimulated cells to determine the role of basal, endogenous Nur77. Conversely, in cells stimulated with various apoptosis-inducing agents, Nur77 plays a role in cell death through both transcription-dependent and independent mechanisms (Figure 2-1). Because of the dual and opposite roles of Nur77 in cell proliferation and death, many studies have been focused on therapeutically targeting Nur77 to impede its oncogenic functions while coaxing it to activate the cellular death program [87]. These efforts would rely on the fact that non-tumor tissue will express Nur77 at much lower levels, making these tissues less responsive to Nur77mediated apoptosis-inducing agents.

Nurrl has been implicated in cancer progression although its cancer-related target genes have not been characterized. Nurr1 knockdown decreases anchorage-independent growth, suggesting that Nurrl plays a role in cell transformation [88, 89]. The protein promotes migration but not overall proliferation in bladder cancer [90], although it does affect cell proliferation in lung and breast cancer [91, 92]. Nurr1 expression is higher in squamous cell carcinoma (SCC) samples than in normal tissues of patients with SCC, and induction of Nurrl expression in SCC leads to increased resistance to 5-fluorouracil [93], suggesting a role for Nurr1 in drug resistance [93, 94]. Additionally, Nurr1 overexpression contributes to protection from doxorubicin-induced apoptosis by diminishing the p53 response [95].

In patients with breast cancer, Nurr1 expression in normal breast epithelium is higher than that in tumor tissue and has been positively correlated with favorable prognosis [92]. Conversely, the same study found that knockdown of Nurr1 in breast cancer cell lines diminished xenograft tumor growth. The different roles of Nurr1 in different tissues point to possible context-dependent effects of Nurr1, which might also depend on the intracellular localization of Nurr1 protein as cytoplasmic expression of Nurr1 in bladder cancer was correlated with decreased patient survival [90]. However, other studies using either stimulated endogenous or overexpressed exogenous Nurrl have not clearly determined its subcellular localization.

Less is known about Nor-1's functions in cancer, although some key findings have been made. For example, $\mathrm{Nr}_{4} \mathrm{al}^{-/-} ; \mathrm{Nr}_{\mathrm{a}} 3^{-/-}$double-knockout mice develop acute myeloid leukemia (AML) with very rapid onset, dying within 2 to 4 weeks [46]. The myeloid cells from these mice have more S- and G2/M-phase populations and fewer annexin $\mathrm{V}$-positive cells than do those of wild-type mice. The decrease in apoptotic cells was attributed to a reduction in extrinsic cell death signaling, as indicated by a decrease in Fas ligand and TRAIL expression. Expression of Nur77 and Nor-1 were dramatically reduced in AML patient samples. Together, these data suggest that these two NR4A receptors can play overlapping tumor suppressive roles in leukemia, as NR4A singleknockout mice do not develop cancer $[96,97]$. The functional redundancy of Nur77 and 


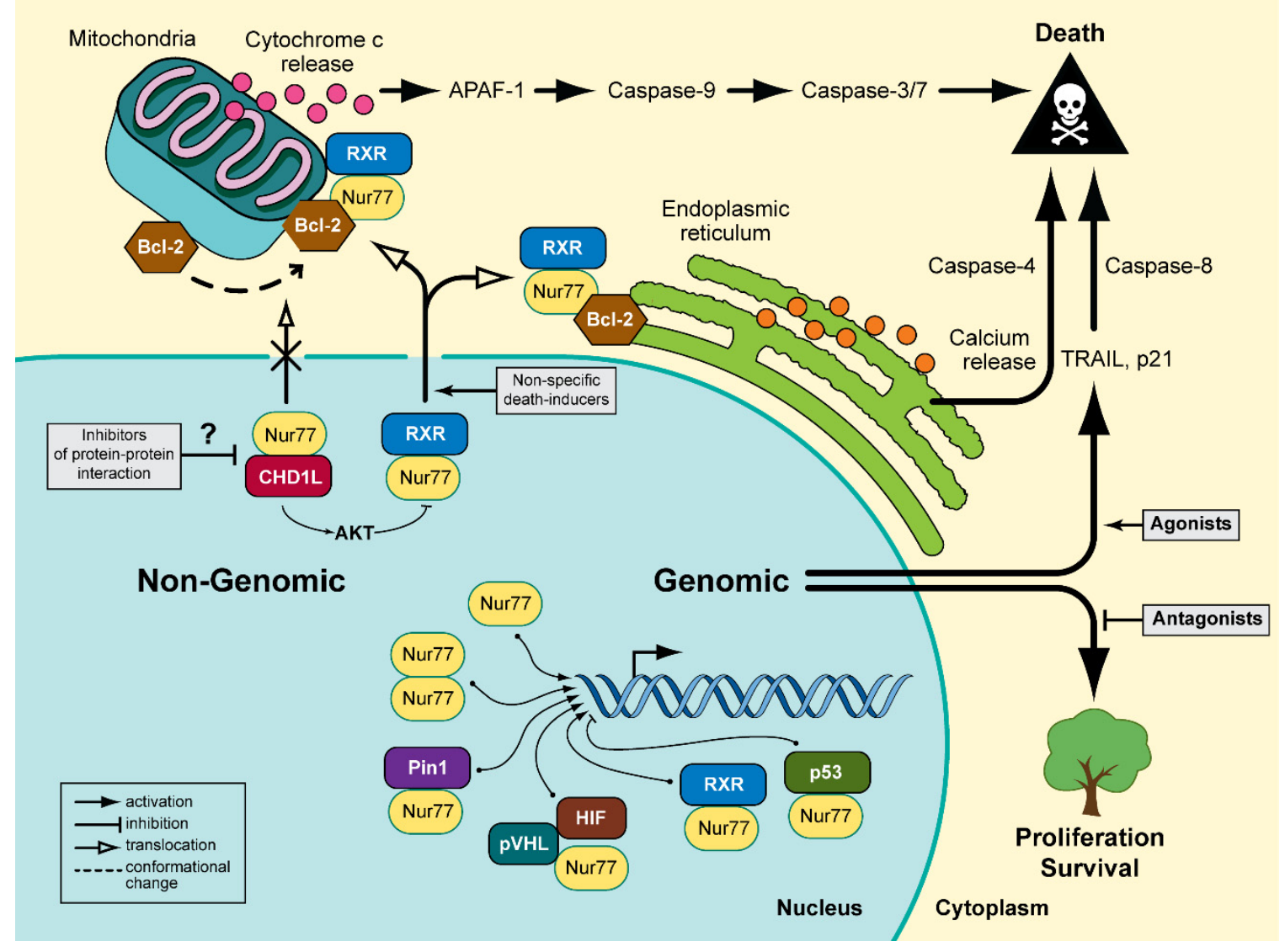

Figure 2-1. Nur77 mediates cell death or survival through localization-dependent and -independent mechanisms.

As a nuclear transcription factor, Nur77 largely promotes cell proliferation and survival through regulation of specific target genes (i.e., cyclin D2, E2F1, survivin, TXNDC5). Additionally, some agonists of Nur77 transactivation are able to mediate transcriptiondependent cell death. A major mechanism of Nur77-mediated cell death is the nuclear export of Nur77-RXR $\alpha$ heterodimers, which is suppressed by the CHDIL oncogene. Cytoplasmic Nur77 can activate mitochondrial- or ER-associated cell death by interacting with membrane-bound Bcl-2. 
Nor-1 was further confirmed in a follow-up study investigating genome-wide transcriptional changes in response to NR4A restoration in AML [98]. Nur77 and Nor-1 shared overlapping gene signatures by regulating $97 \%$ of the same transcripts, and reexpression of either NR4A receptor was able to elicit tumor-suppressive functions by reducing proliferation and increasing apoptosis. Furthermore, NR4A re-expression suppressed MYC and its accompanying oncogenic signature in multiple AML cells.

Another perturbation of Nor-1 function occurs in extraskeletal myxoid chondrosarcoma (EMC). Researchers identified a recurrent translocation of Nor-1 (also called TEC, $\underline{T}$ ranslocated in $\underline{E}$ xtraskeletal $\underline{C}$ hondrosarcoma) with the EWS gene that encodes a novel EWS-TEC fusion protein in EMC tumors [99]. This oncogenic fusion protein binds to and regulates the $\underline{N G F I-\underline{B}} \underline{R}$ esponse $\underline{E}$ lement (NBRE), with 250 -fold greater transactivation capacity than that of wild-type Nor-1 [100]. Because EWS-TEC fusion protein can bind to the NBRE, several studies have been focused on the differential transactivation of target genes of EWS-TEC and Nor-1 [101, 102]. Analysis of 16 EMC tumors showed that 15 cases contained EWS-TEC fusion transcripts [103]. Introduction of the EWS-TEC oncogenic fusion protein into a chondrogenic cell line did not increase the proliferation rate but allowed cells to grow past contact inhibition to form small clusters of cells and increased anchorage-independent growth [104]. Nor-1 also forms fusion proteins with other proteins in EMC $[105,106]$.

\section{NR4A Receptors and MAPK}

\section{MAPK and cancer}

The mitogen-activated protein kinase (MAPK) pathway is induced by a wide range of signals, including growth factors, cytokines, and stress, and is responsible for almost every cell function, including proliferation, differentiation, cell death, and migration. The six different MAPK pathways include extracellular signal-regulated kinase (ERK) 1/2, ERK 3/4, ERK5, ERK 7/8, Jun N-terminal kinase (JNK) 1/2/3, and p38 (ERK6), as reviewed in [107]. Typically, an external signal triggers the signaling cascade through a membrane-bound receptor, followed by recruitment of G-proteins such as Ras, Rac, and Rho and, subsequently, of downstream kinases such as Raf and MAPK kinase kinase (MEKK), which phosphorylate and activate MAPK kinase (MEK). Activated MEKs then phosphorylate and activate MAPKs [107], which will phosphorylate either transcription factors to modulate target gene expression or other kinases to regulate critical cellular events. Because the MAPK pathway is involved in almost every cellular process, it is understandable that dysregulation of this pathway could cause cancer. 


\section{Regulation of NR4A receptors by MAPK}

Phosphorylation of Nur77 by MEK-ERK signaling has been described by several groups. Epidermal growth factor (EGF), an activating signal for ERK2, causes phosphorylation of Nur77 at threonine 142 [108], a phosphorylation site that stabilizes Nur77 [109], consistent with the phosphorylation-mediated stability of other nuclear receptors. Interestingly, EGF enhances the interaction of Nur77 with prolyl isomerase Pin1, and the isomerization of phosphorylated Nur77 requires ERK2- (and JNK1)mediated phosphorylation [110]. This phosphorylation-dependent isomerization prevents degradation of Nur77, thereby increasing its transactivation activity and promoting its pro-mitogenic effect. ERK2 is responsible for phosphorylation of Nur77 at serine 431, a phospho-residue important for both the Nur77-Pin1 interaction and increased transactivation mediated by Pin 1 .

ERK2 activation upon EGF treatment causes Nur77 nuclear localization and prevents its cytosolic induction of apoptosis [111], but the opposite phenotype occurs in T-cells. Inhibiting MEK1 decreases expression of Nur77 in T-cells [112] and prevents its nuclear export and mitochondrial localization [113], demonstrating the role of ERK signaling in Nur77-induced cell death. Ribosomal S6 kinase 2 (RSK2), an effector kinase of MEK-ERK, phosphorylates Nur77 at serine 354 [113,114] and positively regulates Nur77's nuclear export and subsequent mediation of apoptosis. Other groups have reported phosphorylation of Nur77 by ERK5, but not by ERK2, in T-cells that caused increased Nur77 transcription and induction of apoptosis $[115,116]$.

Activation of ERK1/2 signaling is positively associated with the nuclear localization of Nur77 and negatively associated with the ability of fenretinide to induce apoptosis [117]. Inhibiting MEK-ERK and treating with fenretinide enhance the cytosolic localization of Nur77 in fenretinide-resistant HepG2 cells, but activating MEK-ERK prevents this event in fenretinide-sensitive HuH-7 cells, demonstrating a role for ERK signaling in the drug resistance of liver cancer cells. In another study, constitutive signaling through BRAF-MEK-ERK was shown to positively regulate the expression of both Nur77 and Nurr1 via inhibition of BRAF and MEK1/2 [118], implicating this commonly mutated and hyperactive pathway as one of the causes of increased Nur77 levels in cancers. In addition, the p38 MAPK pathway can also modulate the activity of Nurr1. For example, upon activation by apoptosis signal-regulating kinase 1, p38 phosphorylates Nurr1, leading to synthesis of melanin, a pigment important in protecting skin, hair, and eyes from harmful elements [119].

In another study, fibroblast growth factor $8 \mathrm{~b}(\mathrm{FGF}-8 \mathrm{~b})$ induced the expression of all three NR4As in MC3T3-E1 preosteoblastic cells [120]. Furthermore, the effect of FGF-8b was mediated through the MAPK, phosphatidylinositol 3-kinase (PI3K), and protein kinase $\mathrm{C}$ (PKC) pathways. Proliferation of these cells can be increased by FGF$8 \mathrm{~b}$, and overexpression of Nur77 and Nurr1 further enhance this proliferative effect and decrease apoptosis. Therefore, the MAPK pathways regulate the levels, sub-cellular localizations, and activities of the NR4A receptors. 
NR4A Receptors, PI3K-AKT, and mTOR in Proliferation and Survival

\section{PI3K-AKT and cancer}

The PI3K-AKT signaling axis is a major regulator of cell proliferation and survival, acting downstream of growth factors and receptor tyrosine kinases in parallel with MAPK signaling [121]. PI3K heterodimers consist of regulatory and catalytic subunits, which are responsible for PI3K regulation and downstream signaling, respectively. Upon receptor tyrosine kinase activation and recruitment of PI3K to the plasma membrane, the primary function of PI3K is to add a phosphate group to phosphatidylinositol 4,5-bisphosphate ( $\left.\mathrm{PIP}_{2}\right)$, converting it to phosphatidylinositol 3,4,5trisphosphate $\left(\mathrm{PIP}_{3}\right)$ [122] to flip the "on" switch for downstream signaling. $\mathrm{PIP}_{3}$ recruits AKT kinase to the plasma membrane, enabling its phosphorylation and activation by 3 phosphoinositide-dependent protein kinase-1 (PDK1) [123, 124]. Conversely, AKT signaling can be turned off through the phosphatase PTEN, which converts $\mathrm{PIP}_{3}$ back to $\mathrm{PIP}_{2}[125,126]$. AKT can inhibit apoptosis to promote cell survival by phosphorylating and inhibiting both pro-apoptotic Bad [127] and caspase-9 [128]; enhance cell-cycle progression by phosphorylating and inactivating glycogen synthase kinase 3 beta (GSK3B), leading to stabilization of cyclin D1 [129]; and increase cell growth by stimulating the mechanistic target of rapamycin (mTOR) pathway to promote protein synthesis [130].

Alterations in the PI3K-AKT pathway have been identified in various cancer types and include mutations that directly increase PI3K and AKT activity $[121,131]$ and inactivate the tumor suppressor PTEN [132-134]. Mice that are heterozygous for PTEN develop an array of tumor types [135] due to uncontrolled PI3K-AKT signaling. Aberrant AKT signaling can also lead to inactivation of tumor suppressor p53 [136], with the hyperactivation of PI3K-AKT in cancer ultimately leading to increased cell survival and proliferation and contributing to tumor growth, metastasis, and angiogenesis through modulation of downstream effectors, including NR4A receptors.

\section{The interplay of NR4A receptors and PI3K-AKT}

AKT phosphorylates Nur77 [137-139] to negatively regulate its function in mediating cell death. Specifically, AKT phosphorylates human Nur77 at Ser-351 (Ser350 in rats) in the DNA-binding domain [139], a phosphorylation site that inhibits Nur77 transcriptional activity [140]. AKT-mediated phosphorylation of Nur77 occurs in the cytoplasm in a PI3K-dependent manner. More importantly, phosphorylation of Nur77 by AKT decreases the transcriptional activity of Nur77 by 50\%-85\%. Additionally, the other two NR4A members, Nurr1 and Nor-1, also have a similar phosphorylation motif, so it is likely that AKT also phosphorylates Nurr1 and Nor-1, although this has not been investigated. Furthermore, AKT directly inhibits Nur77's DNA binding activity [138]. 
In addition to inhibiting its DNA binding and transcriptional activity, AKT also prevents Nur77 from inducing apoptosis in T-cell hybridomas [138]. To inhibit apoptosis and increase cell survival, AKT phosphorylation of Nur77 can be considered to be a "priming" step for an interaction with 14-3-3 protein, which recognizes the phosphorylated motif near the Ser-351 residue. This protein-protein interaction, which only occurs with wild-type Nur77 following AKT phosphorylation, is similar to AKTmediated phosphorylation of Bad and its subsequent interaction with 14-3-3: both protein-protein interactions prevent the protein (Nur77 or Bad) from interacting with Bcl2 and causing subsequent apoptosis [127]. By using a DNA-binding domain deletion mutant of Nur77 that readily localizes to the mitochondria and induces apoptosis [141], researchers showed that overexpressing AKT blocks mitochondrial association with Bcl2 and causes a diffuse cytoplasmic localization of Nur77 [137]. Because the Nur77 DNAbinding domain deletion mutant lacks the Ser-351 residue, this experiment also showed that AKT can phosphorylate cytoplasmic Nur77 at the N-terminus.

AKT can also act on nuclear Nur77, preventing its nuclear export and subsequent apoptosis $[137,142]$. Overexpression of constitutively active AKT can overcome the effects of MEKK1-induced nuclear export of Nur77, retaining it within the nucleus [142]. The effect of AKT depends on Ser-351 of Nur77: if Ser-351 is replaced with alanine, the Nur77 mutant migrates to and remains in the cytoplasm in response to MEKK1 activation, regardless of AKT status. Inhibition of PI3K-AKT or knockdown of AKT restores Nur77's cytoplasmic localization, whereas PI3K activation by insulin or AKT overexpression efficiently blocks TPA-induced Nur77 nuclear export, cytochrome $c$ release, and apoptosis in gastric cancer cells [137]. In addition to Ser-351, the N-terminal region of Nur77, specifically residues 51-105, are also shown critical for AKT binding and phosphorylation-dependent regulation [137]. AKT's inhibition of Nur77 has also been credited with mediating cisplatin-induced apoptosis in ovarian cancer cells [143].

\section{The interplay of NR4A receptors and mTOR}

AKT acts on multiple downstream proteins, including the mTOR kinase complex (mTORC1). The PI3K-AKT-mTOR axis could be considered to be a single pathway [130] in which AKT activates mTOR to control cell proliferation and growth in response to environmental stimuli by phosphorylating and inactivating the mTOR suppressors tuberous sclerosis protein 1 (TSC1) and TSC2 [144, 145]. Another upstream negative regulator of $\mathrm{mTOR}$, liver kinase $\mathrm{B} 1$ (LKB1), activates AMP-activated protein kinase (AMPK) [146] to suppress the mTORC1 complex [147]. PTEN also negatively regulates mTOR by turning off PI3K-AKT signaling. The major effect of mTOR activation is increased protein synthesis, leading to increased expression of proteins involved in proliferation, survival, and angiogenesis [130].

Nur77 indirectly activates mTOR signaling by attenuating AMPK signaling, and knockdown of Nur77 in non-small cell lung cancer cells decreased proliferation and enhanced apoptosis [148]. Whereas AMPK $\alpha$ phosphorylation was increased and mTOR phosphorylation decreased in that study, AKT phosphorylation status remained 
unchanged, suggesting that the effect on mTOR occurs downstream of AKT. A previous microarray study by the same group demonstrated that sestrin-2 expression increased after knockdown of Nur77 [50]. Sestrin-2, a target gene of p53 [149], serves to activate AMPK signaling. Knowing that Nur77 has been shown to interact with and inhibit p53 [150], the authors further showed that knockdown of Nur77 enhanced sestrin-2-mediated AMPK activation only in cells with wild-type p53. Further support for the role of Nur77 in mTOR activation comes in a report demonstrating Nur77's suppression of AMPK signaling [59], wherein knockdown or overexpression of Nur77 increased or decreased AMPK phosphorylation, respectively. This relationship was not seen in LKB1-null HeLa cells unless LKB1 was cotransfected with Nur77. Furthermore, Nur77-LBD interacts with and sequesters LKB1 in the nucleus, away from cytoplasmic AMPK, leading to decreased AMPK phosphorylation. A subsequent chemical screen found that the small molecule ethyl 2-[2,3,4-trimethoxy-6-(1-octanoyl)phenyl]acetate (TMPA) enhanced AMPK phosphorylation by disrupting the Nur77-LKB1 interaction; this effect was not seen in LKB1-null cells, further confirming LKB1-dependency. These studies demonstrate interesting perspectives of Nur77 in which multiple signaling nodes are interconnected through Nur77, as PI3K-AKT suppresses the pro-apoptotic functions of Nur77 and Nur77 itself enhances downstream mTOR signaling to promote tumor progression, possibly in the context of LKB1 or p53.

\section{NR4A Receptors, Hypoxia, and Angiogenesis}

\section{HIF-1 and cancer}

The hypoxia-inducible factors (HIFs) are a family of transcription factors that mediate the balance of oxygen within tissues, having functions in multiple diseases, both in protective and pathogenic roles [151]. At normoxic conditions (i.e., normal oxygen levels), the HIF-1 $\alpha$ subunit undergoes rapid proteolysis to maintain a low protein level, and the HIF-1 $\beta$ subunit remains at a relatively constant level $[152,153]$. The HIF-1 $\alpha$ subunit is maintained in its suppressed state by hydroxylation of specific prolyl residues $[154,155]$, which promotes an interaction with the von Hippel-Lindau tumor suppressor protein (pVHL) [156], a key protein responsible for targeting HIF-1 $\alpha$ for proteasomal degradation by recruiting ubiquitin ligases [157]. The suppression of HIF-1 $\alpha$ is released under hypoxic conditions (i.e., low oxygen levels) due to the unavailability of oxygen for the hydroxylation step, leading to loss of pVHL recognition of HIF-1 $\alpha$ and an accumulation of HIF-1 $\alpha$ protein. Additionally, a hydroxylation of HIF- $1 \alpha$ by factorinhibiting HIF prevents binding of the coactivator p300 to the transactivation domain of HIF-1 $\alpha$, preventing its transactivation [158]. Once at sufficient levels, HIF-1 $\alpha$ migrates to the nucleus where it forms a heterodimeric transcriptional complex with HIF-1 $\beta$ to regulate the expression of target genes, including those encoding erythropoietin (EPO) [159], vascular endothelial growth factor (VEGF) [160], and proteins involved in glucose uptake and metabolism [161-164]. The HIF-1 transcription factors are able to mediate key processes within hypoxic regions deep within newly established tumor sites to control the balance of oxygen consumption and oxygen delivery. HIF-1 effectively 
controls these processes by regulating the expression of hundreds of genes, many of which are involved in proliferation, metabolic adaption, and angiogenesis $[165,166]$, which are key hallmarks of cancer [167]. As summarized by Semenza [165], loss-offunction and gain-of-function studies have revealed that the HIF-1 target gene products are tumor-promoting and, thus, increase proliferation, angiogenesis, and metastasis in multiple tumor models. Many primary tumors and metastases have increased HIF-1 activation as indicated by increased staining of the HIF-1 protein in tissue samples when compared to adjacent normal tissue [168, 169], and its expression is associated with increased tumor vascularization and aggressiveness [170]. Increased HIF-1 $\alpha$ expression and activity in cancer can be attributed to cancer-associated hypoxia, loss of tumor suppressor function (i.e. pVHL, p53 or PTEN), or contributions of growth factor or oncogene signaling (i.e. PI3K or MAPK), either through blocking degradation or enhancing synthesis of HIF-1 $\alpha$ [166]. Patients with clear cell renal carcinoma and loss of pVHL have enhanced HIF-1 $\alpha$ expression due to the decreased degradation of HIF-1 $\alpha$, defining $\mathrm{pVHL}$ as a tumor suppressor $[171,172]$.

\section{The interplay of NR4A receptors and HIF-1}

Hypoxia increases the expression of all three NR4A family members at both mRNA and protein levels in a HIF-1 $\alpha$-dependent manner [173-177]. HIF-1 $\alpha$, but not HIF-2 $\alpha$, can directly regulate Nur77 and Nor-1 expression by binding to hypoxiaresponse elements in each gene's promoter [173, 174, 176]. Additionally, the Nur77 target gene proopiomelanocortin $(P O M C)$ is induced under hypoxic conditions through HIF-1 $\alpha$-dependent regulation of Nur77. Furthermore, Nur77 itself regulates HIF-1 $\alpha$, implicating this as a possible feedback mechanism in cancer progression. Expression of wild-type but not of dominant negative Nur77 can activate a hypoxia response elementcontaining promoter, increasing nuclear localization of HIF-1 $\alpha[174,178]$. Additionally, when examining this HIF-1 response to Nur77, the HIF-1 target gene VEGF increased at both the mRNA and protein levels, but HIF-1 $\alpha$ increased at only the protein level and underwent attenuated ubiquitination [174, 178], suggesting stabilization of transcriptionally active HIF-1 $\alpha$ by Nur77. Two reports have suggested different Nur77 domains as being requirements for HIF- $1 \alpha$ stabilization and transactivation, either through the N-terminal [174] or ligand-binding domain [178]. These domain-specific stabilizations of HIF-1 $\alpha$ depend on different Nur77 protein interactions, although neither affects HIF-1 $\alpha$ binding with pVHL. The N-terminal domain of Nur77 can block Mdm2 from binding to and degrading HIF-1 $\alpha$ and also leads to decreased Mdm2 expression [174]. However, another group demonstrated that Nur77-LBD can interact with the $\alpha$ domain of $\mathrm{pVHL}$, forcing elongin $\mathrm{C}$ dissociation and blocking $\mathrm{pVHL}$-mediated degradation of HIF-1 $\alpha$ [178]. The fact that pVHL was required for Nur77-mediated stabilization indicates that $\mathrm{pVHL}$ serves as an adaptor protein to form a Nur77-pVHLHIF-1 $\alpha$ complex. Interestingly, Nurr 1 and Nor-1 positively regulate HIF-1 $\alpha$ expression $[175,178]$, and HIF-1 $\alpha$-induced Nor-1 protects endothelial cells exposed to hypoxia, possibly by regulating cellular inhibitor of apoptosis 2 [176]. 
The regulation and function of Nur77 within hypoxic environments may be critical factors to consider and are addressed by a recent report showing crosstalk between Nur77 and $\beta$-catenin signaling during hypoxia [177], in which both were induced and required to positively regulate each other through transcription-independent mechanisms. Although Nur77 is typically considered to be a nuclear protein that regulates target gene expression in the context of cancer, hypoxia-induced Nur77 is highly expressed in the cytoplasm, and this localization is required for stabilization of $\beta$ catenin [177]. This finding is similar to one in bladder cancer patients that indicated cytoplasmic Nurr1 level was correlated with poor prognosis, although other possible factors were not described [90]. Hypoxia can increase AKT phosphorylation in a Nur77dependent manner [177], presenting a unique regulatory mechanism, as Nur77 is phosphorylated by AKT to prevent its mitochondrial localization [137], thus preventing cell death and enhancing its pro-oncogenic functions. The feed-forward loop of Nur77 and $\beta$-catenin promotes cell growth, migration, and invasion and alters epithelial-tomesenchymal transition (EMT) markers [177]. Together, these studies reveal that hypoxia affects both the level and function of NR4A receptors, which in turn play positive feedback roles that affect hypoxia-induced signaling.

\section{NR4A receptors as mediators of VEGF-induced angiogenesis}

Given the reported roles of the NR4A receptors in hypoxia, a reasonable hypothesis might be that hypoxia-induced upregulation of NR4A receptors will positively regulate HIF-1 $\alpha$, thus increasing HIF-1-regulated genes and promoting survival, angiogenesis, and tumor promotion. The anti-metabolite 6-mercaptopurine (6-MP), known to increase NR4A transactivation through its AF-1 domain [179], induces the expression of all three NR4A members, HIF-1 $\alpha$, and VEGF and increases the stability, nuclear localization, and transactivation of HIF-1 $\alpha$ protein in a Nur77-dependent manner [175]. The effects of 6-MP on NR4A and HIF-1 $\alpha$ depend on p44/p42 ERK phosphorylation and can be abolished using a MEK inhibitor, a previously reported regulatory mechanism of Nur77 in hypoxia [174]. Furthermore, 6-MP enhances the HIF$1 \alpha$ response in endothelial cells and promotes capillary tube formation [175], indicating that induction of Nur77 and its regulation of HIF-1 $\alpha$ can promote angiogenesis.

One main mediator of angiogenesis is VEGF, which is induced by deoxycholic acid (DCA) through enhancing the expression of Nur77 in colon cancer cells [180]. VEGF stimulation of cells rapidly induces expression of all three NR4A members [181184], which mediate VEGF-induced effects on proliferation and angiogenesis. Similar to VEGF, Nur77 expression increases the proliferative rate of endothelial cells and protects cells from apoptosis [183], suggesting that VEGF exerts its effect by upregulating Nur77 expression. Nur77 knockdown increases apoptosis, which cannot be rescued by addition of VEGF, further suggesting that Nur77 operates downstream of VEGF in this context. In addition, the effect of VEGF depends on Nur77, and both VEGF and Nur77 induce the expression of the cell cycle-related genes cyclin A, cyclin D1, proliferating cell nuclear antigen $(P C N A)$, and $\mathrm{E} 2 \mathrm{~F}$ [183]. Inactivation of Nur77 reduces capillary tube formation, and mutants lacking the DNA-binding domain undergo no tube formation [183]. 
Compared to wild-type mice, Nur $77^{-/-}$mice form fewer xenograft tumors, with reduced angiogenesis within the tumors [183]. These data suggest that VEGF-induced angiogenesis is mediated through Nur77.

Induction of Nor-1 by VEGF has been attributed to VEGF receptor 2 (VEGFR-2), and its expression is modulated at the Nor-1 promoter through CBP [182]. Knockdown of Nor-1 in endothelial cells attenuates DNA synthesis and progression of cells into S-phase following VEGF stimulation, suggesting that Nor-1 mediates the effects of VEGF and confirming a previous finding of Nor-1's role in vascular smooth muscle proliferation [185]. Similarly, VEGF-induced Nurr1 expression occurs rapidly, within 1 hour, and is mediated at the promoter level by NF- $\mathrm{KB}$ and CREB response elements [184]. Upon VEGF stimulation, CREB becomes phosphorylated and binds to the Nurr1 promoter, and this action can be blocked by inhibiting protein kinase D. Nurr1 knockdown can inhibit VEGF-mediated proliferation, migration, and in vivo angiogenesis [184]. These studies demonstrate that NR4A receptors are critical mediators of VEGF-mediated signaling.

\section{NR4A Receptors, p53, and Cell Death}

\section{p53 and cancer}

As the main arbitrator of determining cell cycle progression, DNA repair, and apoptosis, the tumor suppressor $\mathrm{p} 53$ is a central hub in regulating cell fate $[186,187]$. In response to a stress stimulus, such as DNA damage, p53 is quickly induced. Induction of p53 typically occurs at the protein level through inhibition of p53 degradation, known as derepression, by the blocking of a critical ubiquitination by the E3 ligase Mdm2 and, ultimately, the enhancement of p53 protein stability. With increased p53 protein levels, the cell's fate can now be regulated by tetramerization of $\mathrm{p} 53$ proteins and transcription of target genes. For example, p53 can drive the expression of cyclin-dependent kinase p21 [188, 189] and Gadd45 [190] to block cell-cycle progression, allowing the cell enough time to undergo DNA repair to correct any lesions. If the damage received proves to be too extensive, then p53 can initiate the cell death program through induction of genes such as Puma [191], Noxa [192], and Bax [193] to prevent the outgrowth of cells with damaged genomes. Additionally, p53 can execute the cell death program through DNA-binding-independent mechanisms by forming complexes with other signaling molecules such as B-cell lymphoma 2 (Bcl-2) at the mitochondrial membrane, which compromises the integrity of the outer mitochondrial membrane, resulting in cytochrome c release [194]. Through these multiple mechanisms of halting cell expansion, p53 serves as a critical tumor suppressor to prevent the formation of malignant lesions.

Since the discovery of $\mathrm{p} 53$, an overwhelming amount of reports have suggested that somatic p53 mutations occur in at least half of all cancers, with higher frequencies in certain malignancies, making it the most commonly mutated gene in cancer [195].

Mutations of p53, which commonly occur in its DNA-binding domain, serve to hijack its function as a tumor suppressor, making cells vulnerable to malignant transformation. 
Also, because many p53 mutations occur in the DNA-binding domain, mutated p53, acting in a dominant-negative manner, is still able to form tetramers with wild-type p53 protein to render them inactive [196]. Accumulating evidence also points to oncogenic functions of mutated p53 [197]. Additionally, in cancers with wild-type p53, the function of p53 can still be altered through overexpression of the negative regulator Mdm2 [198]. The importance of p53 in tumor suppression is evident in studies of p53 knockout mice in which all mice lacking p53 eventually succumb to disease, mostly due to sarcomas and lymphomas [199].

\section{The interplay of NR4A receptors and p53}

Both Nur77 and Nurr1 interact with p53 and regulate critical p53-dependent signaling, which could at least partially explain the oncogenic functions of NR4A receptors. On the heels of a finding that Nur77 could mediate Mdm2 degradation to promote HIF-1 $\alpha$ stabilization [174] despite the lack of a Nur77-Mdm2 interaction, a direct interaction of Nur77 with p53 was demonstrated [150] that could explain this negative regulation of Mdm2. The Nur77-p53 interaction leads to a blockade of p53 acetylation, resulting in loss of p53-dependent transactivation and subsequent decreased expression of the target genes Mdm2 and cyclin-dependent kinase inhibitor $\mathrm{p} 21$. Interestingly, Nur77 can enhance p53-dependent apoptosis with and without UV irradiation, suggesting non-genomic regulation within the Mdm2-p53 axis. Ubiquitination of $\mathrm{p} 53$ by Mdm2 is also obstructed by Nur77, enhancing its stability. The results of these studies suggest that the pool of available $\mathrm{p} 53$ protein might play an important role in its transcriptional regulation, protection from Mdm2-mediated destruction, and enhancement of apoptosis conferred by Nur77. More recent findings show that the Nur77-mediated enhancement of p53-dependent apoptosis is due to phosphorylation of Nur77 by DNAdependent protein kinase (DNA-PK) [200]. This phosphorylation of Nur77 enhances that of $\mathrm{p} 53$ by DNA-PK and, ultimately, increases the potential for induction of apoptosis upon DNA damage.

Similarly, Nurr1 interacts with p53 and inhibits p53-dependent apoptosis by inhibiting transactivation [95]. Nurr1 interacts with the C-terminal domain of p53 to attenuate doxorubicin-induced expression of the proapoptotic protein Bax, and cells lacking p53 do not exhibit doxorubicin resistance in the presence of Nurr1. The effects of Nurr1 on p53 are attributed to a reduction in the tetramerization of p53, which is required for its transcriptional activity. Although Nurrl also has roles in DNA-PK-mediated DNA repair [201], the role of a Nurr1-p53 interaction in this process is unknown. Interestingly, a recent study found Nurr 1 expression to be inversely correlated with p53 expression in primary breast cancer tissues [92]. The effects of p53 interaction with Nurr1, and Nur77 likewise, have not been addressed, and it remains to be determined whether p53 can interfere with the oncogenic effects of the NR4A receptors through direct protein interactions or other signaling mechanisms.

NR4A receptors not only affect the immediate responses of $\mathrm{p} 53$ but also affect other downstream pathways in a p53-dependent context. One such pathway is the 
AMPK-mTOR axis. As discussed in section 4.3, Nur77 can indirectly activate mTORC1 signaling by inhibiting p53 [148]. In p53 wild-type cells, but not in p53-null cells, Nur77 inhibited p53-mediated transactivation of the sestrin-2 promoter and subsequent expression of sestrin-2, a known target of p53 that inhibits mTOR signaling [149].

\section{Nur77 in cell death}

An intricate review by Moll and colleagues [202] draws parallels between p53 and Nur77 as cell death mediators that act through the intrinsic cell death pathway. Regardless of the route in which p53 activates cell death (i.e., transcription-dependent or -independent), the ultimate outcome is a mitochondria-induced cell death either through p53 target genes such as PUMA or $B A X$ or through direct interactions with other molecules such as Bcl-2 at the mitochondrial outer membrane. Similarly, increasing evidence points to multiple mechanisms of Nur77-mediated cell death through nuclear and cytosolic Nur77 functions (Figure 2-1). A well-characterized mechanism of Nur77mediated cell death is that in which Nur77 translocates from the nucleus to begin the apoptosis cascade, independently of transactivation, in response to certain death-inducing compounds [56, 203]. Nur77 heterodimerizes with retinoid X receptor (RXR) alpha and translocates from the nucleus to the cytosol, where the complex can target the mitochondria [204-206]. Bcl-2, a protein that is anti-apoptotic under most conditions, serves as a receptor on the mitochondrial outer membrane and is the downstream effector of Nur77 through an interaction with its ligand-binding domain [207]. Anti-apoptotic Bcl-2 is converted to a pro-apoptotic molecule through a conformational change of its Bcl-2 homology (BH) domains after Nur77 binding [207]. The conformational change exposes the buried pro-apoptotic $\mathrm{BH} 3$ domain of $\mathrm{Bcl}-2$, resulting in a release of cytochrome $c$ [141] and further activation of apoptosis. Deletion of the BH3 domain of Bcl-2 inhibits Nur77-mediated apoptosis. Another report suggests that Nur77 can mediate stress-induced apoptosis by targeting Bcl-2 at the endoplasmic reticulum (ER), leading to a release and depletion of ER calcium and activation of caspase-4 and -8 [208]. Therefore, cytosolic Nur77, by interacting with Bcl-2 either at the mitochondrial outer membrane or ER, is pro-apoptotic.

Although Nur77 exerts its pro-apoptotic effect largely through a translocationdependent mechanism, it also regulates specific target genes involved in cell cycle regulation, survival, and apoptosis. Therefore, small-molecule agonists [49-55] or antagonists [47-49] of Nur77 could be useful in modulating its transcription factor functions. One group has identified methylene-substituted diindolylmethanes (DIMs) as being agonists or antagonists of Nur77 and has shown that in vitro and in vivo treatment of colon, pancreatic, and bladder cancer cells with DIM-C-pPhOCH 3 activates Nur77 through its ligand-binding domain [49], leading to growth inhibition, cell cycle arrest, and/or cell death [49-53]. Treatment with DIM-C-pPhOCH 3 causes a Nur77-dependent increase in TNF-related apoptosis-inducing ligand (TRAIL/TNFSF10), p21, and other genes, implicating DIM-C-pPhOCH3 in regulating the cell cycle and apoptosis [49-52] (Table 2-1). The DIM-C-pPhOCH3-mediated effects are independent of Nur77's translocation to mitochondria, and Nur77 remains localized within the nucleus. TRAIL is 
Table 2-1. Summary of gene expression changes after treatment with NR4A agonists or antagonists.

\begin{tabular}{|c|c|c|c|c|c|c|c|c|c|}
\hline \multirow[b]{2}{*}{ Symbol } & \multirow[b]{2}{*}{ Gene name } & \multicolumn{2}{|c|}{ Compound } & \multicolumn{2}{|c|}{ Detection } & \multirow[b]{2}{*}{$\begin{array}{l}\text { NR4A- } \\
\text { dependent? }\end{array}$} & \multirow[b]{2}{*}{ Direct? } & \multirow[b]{2}{*}{ Cancer type } & \multirow[b]{2}{*}{ Ref } \\
\hline & & Name & Action & Direction & Method & & & & \\
\hline \multirow{2}{*}{$A T F 3$} & \multirow{2}{*}{$\begin{array}{l}\text { Activating } \\
\text { transcription factor } 3\end{array}$} & \multirow{2}{*}{$\begin{array}{l}\text { DIM-C- } \\
\mathrm{pPhOCH}_{3}\end{array}$} & \multirow{2}{*}{ Agonist } & \multirow{2}{*}{ Increased } & $\mathrm{M}, \mathrm{G}$ & No & n.d. & Colon & 51 \\
\hline & & & & & $\mathrm{M}, \mathrm{G}, \mathrm{P}$ & Yes; Nur77 & n.d. & Pancreatic & 53 \\
\hline ATF4 & $\begin{array}{l}\text { Activating } \\
\text { transcription factor } 4\end{array}$ & $\begin{array}{l}\text { DIM-C- } \\
\text { pPhOH }\end{array}$ & Antagonist & Increased & $\mathrm{P}$ & Yes; Nur77 & n.d. & Pancreatic & 48 \\
\hline$B C L 2$ & B-cell lymphoma 2 & $\begin{array}{l}\text { DIM-C- } \\
\text { pPhOH }\end{array}$ & Antagonist & Decreased & $\mathrm{P}$ & n.d. & n.d. & $\begin{array}{l}\text { Pancreatic, } \\
\text { Lung }\end{array}$ & 47,148 \\
\hline BIRC5 & $\begin{array}{l}\text { Baculoviral IAP } \\
\text { repeat containing } 5 \\
\text { (Survivin) }\end{array}$ & $\begin{array}{l}\text { DIM-C- } \\
\text { pPhOH }\end{array}$ & Antagonist & Decreased & $\mathrm{G}, \mathrm{P}, \mathrm{R}, \mathrm{C}$ & Yes; Nur77 & Yes & $\begin{array}{l}\text { Pancreatic, } \\
\text { Lung }\end{array}$ & 47,148 \\
\hline$B R E$ & $\begin{array}{l}\text { Brain and } \\
\text { reproductive organ- } \\
\text { expressed }\end{array}$ & $\begin{array}{l}\text { Cytosporone } \\
\text { B }\end{array}$ & Agonist & Decreased & $\mathrm{M}, \mathrm{G}, \mathrm{P}, \mathrm{R}, \mathrm{C}$ & Yes; Nur77 & Yes & Gastric & 54 \\
\hline CDKN1A & $\begin{array}{l}\text { Cyclin-dependent } \\
\text { kinase inhibitor } 1 \mathrm{~A}\end{array}$ & $\begin{array}{l}\text { DIM-C- } \\
\text { pPhOCH}_{3}\end{array}$ & Agonist & Increased & $\mathrm{M}, \mathrm{G}, \mathrm{P}, \mathrm{R}, \mathrm{C}$ & Yes; Nur77 & Yes & $\begin{array}{l}\text { Pancreatic, } \\
\text { Bladder }\end{array}$ & $50,52,53$ \\
\hline & $(\mathrm{p} 21, \mathrm{Cip} 1)$ & DIM-C-Ph & Agonist & Increased & G & n.d. & n.d. & Pancreatic & 52 \\
\hline DDIT3 & $\begin{array}{l}\text { DNA-damage- } \\
\text { inducible transcript } 3 \\
(\mathrm{CHOP})\end{array}$ & $\begin{array}{l}\text { DIM-C- } \\
\text { pPhOH }\end{array}$ & Antagonist & Increased & $\mathrm{G}, \mathrm{P}$ & Yes; Nur77 & n.d. & Pancreatic & 48 \\
\hline$M Y C$ & $\begin{array}{l}\text { V-Myc avian } \\
\text { myelocytomatosis } \\
\text { viral oncogene } \\
\text { homolog (c-Myc) }\end{array}$ & $\begin{array}{l}\text { DIM-C- } \\
\text { pPhOH }\end{array}$ & Antagonist & Decreased & $\mathrm{P}$ & n.d. & n.d. & Lung & 148 \\
\hline & Cystathionase & DIM-C- & & & $\mathrm{M}, \mathrm{G}$ & No & n.d. & Colon & 51 \\
\hline $\mathrm{CTH}$ & $\begin{array}{l}\text { (cystathionine } \\
\text { gamma-lyase) }\end{array}$ & $\mathrm{pPhOCH}_{3}$ & Agonist & Increased & $\mathrm{M}, \mathrm{G}$ & Yes; Nur77 & n.d. & $\begin{array}{l}\text { Bladder, } \\
\text { Pancreatic }\end{array}$ & 50,53 \\
\hline DUSP1 & $\begin{array}{l}\text { Dual specificity } \\
\text { phosphatase } 1\end{array}$ & $\begin{array}{l}\text { DIM-C- } \\
\mathrm{pPhOCH}_{3}\end{array}$ & Agonist & Increased & $\mathrm{M}, \mathrm{G}$ & Yes; Nur77 & n.d. & Pancreatic & 53 \\
\hline$E G F R$ & $\begin{array}{l}\text { Epidermal growth } \\
\text { factor receptor }\end{array}$ & $\begin{array}{l}\text { DIM-C- } \\
\text { pPhOH }\end{array}$ & Antagonist & Decreased & $\mathrm{P}$ & n.d. & n.d. & Lung & 148 \\
\hline
\end{tabular}


Table 2-1. (Continued).

\begin{tabular}{|c|c|c|c|c|c|c|c|c|c|}
\hline \multirow[b]{2}{*}{ Symbol } & \multirow[b]{2}{*}{ Gene name } & \multicolumn{2}{|c|}{ Compound } & \multicolumn{2}{|c|}{ Detection } & \multirow[b]{2}{*}{$\begin{array}{l}\text { NR4A- } \\
\text { dependent? }\end{array}$} & \multirow[b]{2}{*}{ Direct? } & \multirow[b]{2}{*}{ Cancer type } & \multirow[b]{2}{*}{ Ref } \\
\hline & & Name & Action & Direction & Method & & & & \\
\hline$F A S L G$ & $\begin{array}{l}\text { Fas ligand (TNF } \\
\text { superfamily, member } \\
6)\end{array}$ & $\begin{array}{l}\text { DIM-C- } \\
\mathrm{pPhOCH}_{3}\end{array}$ & Agonist & Increased & $\mathrm{P}$ & Yes; Nur77 & n.d. & Pancreatic & 53 \\
\hline$G D F 15$ & $\begin{array}{l}\text { Growth } \\
\text { differentiation factor } \\
15 \text { (NAG-1) }\end{array}$ & $\begin{array}{l}\text { DIM-C- } \\
\mathrm{pPhOCH}_{3}\end{array}$ & Agonist & Increased & $\mathrm{M}, \mathrm{G}$ & No & n.d. & $\begin{array}{l}\text { Bladder, } \\
\text { Pancreatic }\end{array}$ & 50,53 \\
\hline$P D C D 1$ & $\begin{array}{l}\text { Programmed cell } \\
\text { death } 1\end{array}$ & $\begin{array}{l}\text { DIM-C- } \\
\mathrm{pPhOCH}_{3}\end{array}$ & Agonist & Increased & $\mathrm{M}, \mathrm{G}$ & Yes; Nur77 & n.d. & Colon & 51 \\
\hline NUPR1 & $\begin{array}{l}\text { Nuclear protein, } \\
\text { transcriptional } \\
\text { regulator } 1(\mathrm{p} 8)\end{array}$ & $\begin{array}{l}\text { DIM-C- } \\
\mathrm{pPhOCH}_{3}\end{array}$ & Agonist & Increased & $\mathrm{M}, \mathrm{G}$ & Yes; Nur77 & n.d. & Bladder & 50 \\
\hline \multirow{2}{*}{ SESN2 } & \multirow{2}{*}{ Sestrin 2} & $\begin{array}{l}\text { DIM-C- } \\
\mathrm{pPhOCH}_{3}\end{array}$ & Agonist & Increased & $\mathrm{M}, \mathrm{G}$ & Yes; Nur77 & n.d. & Bladder & 50 \\
\hline & & $\begin{array}{l}\text { DIM-C- } \\
\text { pPhOH }\end{array}$ & Antagonist & Increased & $\mathrm{G}, \mathrm{P}, \mathrm{R}, \mathrm{C}$ & Yes; Nur77 & No & Lung & 148 \\
\hline \multirow{4}{*}{ TNFSF 10} & \multirow{4}{*}{$\begin{array}{l}\text { Tumor necrosis } \\
\text { factor (ligand) } \\
\text { superfamily, member } \\
10 \text { (TRAIL) }\end{array}$} & $\begin{array}{l}\text { DIM-C- } \\
\mathrm{pPhOCH}_{3}\end{array}$ & Agonist & Increased & $\mathrm{G}, \mathrm{P}$ & Yes; Nur77 & n.d. & $\begin{array}{l}\text { Pancreatic, } \\
\text { Colon, } \\
\text { Bladder }\end{array}$ & $\begin{array}{l}50,49,51, \\
53\end{array}$ \\
\hline & & $\begin{array}{l}\text { DIM-C- } \\
\mathrm{pPhCH}_{3}\end{array}$ & Agonist & Increased & $\mathrm{P}$ & n.d. & n.d. & Pancreatic & 49 \\
\hline & & DIM-C-Ph & Agonist & Increased & $\mathrm{G}, \mathrm{P}$ & n.d. & n.d. & $\begin{array}{l}\text { Pancreatic, } \\
\text { Colon, } \\
\text { Bladder }\end{array}$ & $50,49,51$ \\
\hline & & $\begin{array}{l}\text { DIM-C- } \\
\mathrm{pPhCl}\end{array}$ & Agonist & Increased & $\mathrm{G}, \mathrm{P}$ & Yes; Nurr1 & n.d. & Bladder & 209 \\
\hline$T X N D C 5$ & $\begin{array}{l}\text { Thioredoxin domain } \\
\text { containing } 5\end{array}$ & $\begin{array}{l}\text { DIM-C- } \\
\text { pPhOH }\end{array}$ & Antagonist & Decreased & $\mathrm{M}, \mathrm{G}, \mathrm{P}, \mathrm{R}, \mathrm{C}$ & Yes; Nur77 & Yes & Pancreatic & 48 \\
\hline$X B P 1$ & $\begin{array}{l}\text { X-box binding } \\
\text { protein } 1\end{array}$ & $\begin{array}{l}\text { DIM-C- } \\
\mathrm{pPhOH}\end{array}$ & Antagonist & Increased & $\mathrm{P}$ & Yes; Nur77 & n.d. & Pancreatic & 48 \\
\hline
\end{tabular}

Detection key: $\mathrm{M}=$ microarray, $\mathrm{G}=$ gene, $\mathrm{P}=$ protein, $\mathrm{R}=$ promoter reporter, $\mathrm{C}=$ chromatin $\mathrm{IP}$; $\mathrm{n} . \mathrm{d}$. = not determined 
implicated in the extrinsic pathway of apoptosis, a cell death pathway that bypasses the intrinsic mitochondrial pathway, further supporting a translocation-independent mechanism. As expected, cleavage of caspase- 8 is detected after treatment with DIM-C$\mathrm{pPhOCH}_{3}[49-51,53]$. Another compound in this series of DIM analogs, DIM-C-pPhOH, is described as a Nur77 antagonist, capable of inhibiting both basal and agonist-induced Nur77 transactivation through its N-terminal domain [47-49, 52]. Interestingly, DIM-C$\mathrm{pPhOH}$ can also inhibit in vitro and in vivo cancer cell growth and induce apoptosis [4749, 148], mimicking the effects seen after RNAi-mediated knockdown of Nur77, primarily through a Nur77-dependent regulation of target gene BIRC5/Survivin (Table 2-1). Nur77 knockdown or DIM-C-pPhOH treatment also cause morphological changes indicative of ER stress, which are accompanied by upregulation of ER stress genes and proteins (Table 2-1) and downregulation of the Nur77 target gene TXNDC5, a gene responsible for maintaining proper ROS levels [48]. The structure-dependent effects of C-DIMs on Nur77-regulated pro-apoptotic and anti-apoptotic genes, although not completely straightforward, are attributed to interactions with specific cofactors (i.e., p300) and other transcription factors (i.e., Sp1) [47, 52], but other genes are regulated through direct NBRE- and NuRE-binding sites.

Another DIM analog, DIM-C-pPhCl, was found to be a Nurr1-specific agonist [209]. This compound can inhibit TRAIL induction, apoptosis, and proliferation of bladder cancer cell lines, can block in vivo tumor growth, and can increase overall survival. These data suggest that Nurr1, similar to Nur77, may regulate both proliferative and survival genes (or death-inducing genes) depending on specific stimuli or structuredependent small-molecule features and that agonists or antagonists could be developed to regulate this orphan receptor.

The small-molecule natural product cytosporone B (Csn-B) and its analogs are also ligands for the Nur77 ligand-binding domain [54, 55]. Csn-B stimulates Nur77's transcriptional activity through an interaction with Tyr-453 without affecting the activity of other NRs. A critical hydrogen bond is formed between a hydroxyl group of Csn-B (and its analogs) and Nur77 at Tyr-453 [54]. The series of analogs depend upon Nur77 in mediating apoptosis, which is induced by the translocation of Nur77 to the mitochondria after activation of the intrinsic pathway of apoptosis [55]. Csn-B and analogs suppress the expression of brain and reproductive organ-expressed protein (BRE), an anti-apoptotic protein. The promoter of $B R E$ contains a binding site for Nur77, suggesting that modulation of nuclear Nur77 may suppress genes containing NBRE or NuRE in their promoter, possibly by recruiting corepressors (i.e., nuclear receptor co-repressor-1). Nur77 can also upregulate $B R E$ in colon cancer cells after DCA treatment [180]. 


\section{NR4A, Wnt, and $\beta$-catenin}

\section{Wnt and cancer}

The roles of Wnt signaling in cancer have been previously reviewed [210, 211]. Wnt binds to and activates a Frizzled receptor, which then interacts with the intracellular Dishevelled to activate downstream events, including the canonical (i.e., $\beta$-catenindependent) and non-canonical Wnt pathways [211]. In the canonical pathway, $\beta$-catenin accumulates within the cytoplasm and translocates to the nucleus, where it acts as a transcriptional coactivator of the TCF/LEF family of transcription factors. TCF/LEF then targets genes involved in cell proliferation, stem cell maintenance, and differentiation. These target genes include those of cyclin D1 and c-Myc, which are required for the transition from $\mathrm{G} 1$ to $\mathrm{S}$ phase in the cell cycle. In the absence of Wnt signaling, $\beta$-catenin is normally degraded by a complex of proteins including Axin, adenomatosis polyposis coli (APC), Glycogen synthase kinase $3 \beta$ (GSK3 $\beta$ ), and casein kinase $1 \alpha(\mathrm{CK} 1 \alpha)$. These proteins mediate phosphorylation of $\beta$-catenin, leading to ubiquitination and subsequent proteasomal degradation, thus preventing $\beta$-catenin from accumulating in the cytoplasm and performing its coactivator function.

Dysregulation of Wnt signaling has been linked to cancer development [210]. Wnt 1 ligand is a proto-oncogene in a mouse model of breast cancer. Multiple signaling dysregulations lead to elevated $\beta$-catenin, which is strongly correlated with poor prognosis of breast cancer patients, and have been implicated in other cancers such as colorectal cancer, melanoma, prostate cancer, lung cancer, glioblastoma, esophageal cancer, ovarian cancer, and familial adenomatous polyposis. The initial consensus was that increased Wnt signaling always correlates with negative patient outcomes, however recent evidence shows otherwise. Enhanced Wnt signaling can either promote or inhibit cancer formation and progression, and this is strongly dependent on the type and stage of cancer.

\section{The interplay of NR4A receptors and Wnt signaling}

Nuclear receptors, including the NR4A receptors, modulate the Wnt pathway $[212,213]$. Nurr1 inhibits Wnt signaling by blocking $\beta$-catenin transactivation in both 293F and MC3TC-E1 osteoblastic cells. A similar observation was made in U2OS osteosarcoma cells in which the NR4A receptors block the transcriptional activity of $\beta$ catenin through a mechanism involving the DNA-binding domain of the NR4A receptors [213]. In addition, Nur77 promotes $\beta$-catenin degradation in the cytoplasm and inhibits tumor formation in vivo through transcriptional inhibition of the Wnt pathway [84, 214]. This finding would seem contradictory because Nur77 is overexpressed in most solid tumors; however, analysis of tissue samples from patients with colon cancer revealed that Nur77 is hyperphosphorylated by GSK-3 $\beta$, which may impede its inhibition of the Wnt pathway [214]. Conversely, the NR4A receptors can indirectly increase $\beta$-catenin in melanoma cells. Nur77 targets $\mathrm{CBP} / \mathrm{p} 300$-interacting transactivator 1 (CITED1) and 
Nur77/Nurr1 targets Dishevelled-binding antagonist of beta-catenin 1 (DACT1), both of which are negative regulators of the Wnt pathway. CITED1 inhibits $\beta$-catenin transactivation, and DACT1 interacts with the Wnt activator Dishevelled to promote its degradation, leading to inhibition of the Wnt pathway [118]. Therefore, NR4A receptors inhibit CITED1 and DACT1 to increase Wnt activity.

Alternatively, the Wnt pathway can either upregulate or repress the NR4A receptors depending on the cellular context. In $293 \mathrm{~F}$ cells, $\beta$-catenin and Nurr 1 directly bind, disrupting an interaction with the corepressor Lef-1. This enables Nurr1 and $\beta$ catenin to activate their transcriptional targets. This interaction is important for normal neuron development and the survival of dopaminergic neurons [84, 213]. Nur77 can also be upregulated upon the addition of the colon carcinogen DCA, which stabilizes $\beta$ catenin and allows it to form a transcriptional complex with AP-1 that can then bind to the Nur77 promoter to enhance transcription of Nur77 [84, 213, 214]. Conversely, overexpression of $\beta$-catenin in U2OS and HeLa cells inhibits NR4A transcriptional activity through a mechanism involving the ligand-binding domain of the NR4A receptors [213].

As discussed in section 5.2, a positive feedback loop between Nur77 and $\beta$ catenin has been identified under hypoxic conditions in colorectal cancer cells [177]. $\beta$ catenin induces Nur77 expression through HIF-1 $\alpha$. However, Nur77 can increase $\beta$ catenin's protein levels by increasing its half-life in the cytoplasm. Furthermore, the growth, migration, and invasion of colorectal cancer cells increase upon overexpression of $\beta$-catenin or Nur77, and these effects are further enhanced when $\beta$-catenin and Nur77 are coexpressed. The authors of these findings argued that previous studies on the interaction between Nur77 and $\beta$-catenin are conflicting because of the normoxic conditions used in those studies and that it is, therefore, more realistic to perform these experiments under hypoxic conditions, which more closely mimic the environment of a tumor. Overall, it is clear that the tissue type and environmental conditions play an important role in how NR4A receptors interact with the Wnt pathway.

\section{CHD1L Oncogene and Nur77}

\section{CHD1L and cancer}

Chromodomain helicase/ATPase DNA binding protein 1-like, or CHD1L, is a member of the Snf2-like family of chromatin remodelers and modifiers [215]. Unlike other members of this family, CHD1L does not contain a chromodomain that recognizes methylated histone tails but, instead, harbors a macro domain containing a Poly (ADPribose) (PAR)-binding element [216], which allows binding with Parp1 [217, 218]. Several groups have shown that CHD1L has macro domain-dependent ATPase activity in the presence of DNA and nucleosomes that is enhanced by Parp1 [217, 218]. CHD1L also interacts with proteins involved in DNA repair in a Parpl-dependent manner and is recruited to DNA damage break points through its macro domain [217, 218]. In addition 
to its function in chromatin remodeling and DNA repair, CHD1L also has DNA-binding and transcription factor capabilities [219]. Confirmed target genes of CHD1L include ARHGEF9 [219], TCTP [220] and SPOCK1 [221].

The CHDIL gene, also called ALC1 (Amplified in Liver Cancer 1), is an oncogene, residing in the frequent 1q21 amplicon found in some solid tumors [222], including hepatocellular carcinoma (HCC) [223, 224]; amplification of the 1q21 locus has been found in 58\%-78\% of HCC cases [224, 225]. Gain-of-function and loss-offunction studies have confirmed the role of CHD1L as an oncogene, having the ability to enhance in vitro cell transformation and in vivo tumor formation and tumor size, which can be attributed to its ability to promote the G1/S phase transition [224]. In addition to its growth-promoting effects, CHD1L can protect cells from apoptosis [224] and 5fluorouracil [226]. Analyses of patient samples revealed that approximately $50 \%$ of patients with HCC have CHD1L overexpression [224, 226] and that $68 \%$ of metastatic tumor sites have higher levels of CHD1L than are found in the matching primary tumors [219]. Indeed, overexpression of CHD1L is associated with resistance to chemotherapy in patients with HCC [226]. Studies of CHD1L-transgenic mice further demonstrate the oncogenic ability of CHD1L, with about $25 \%$ of mice forming spontaneous tumors, including some cases of HCC [227].

The effects of CHD1L on oncogenesis can be attributed to its transcription factor function and target genes. The first target gene identified for CHD1L was ARHGEF9 [219], a guanine nucleotide exchange factor that activates Cdc42, which is a GTPase involved in epithelial-to-mesenchymal transition (EMT) and metastasis [228]. Indeed, CHD1L overexpression can induce an AHGEF9-Cdc42-dependent EMT, resulting in

increased in vivo tumor invasiveness and metastasis. Target gene TCTP is overexpressed in about $40 \%$ of HCC patient samples and is associated with advanced tumor stage; its overexpression increases in vivo tumor formation via faster mitotic exit and cell division [220]. Similarly, CHD1L target gene SPOCK1 is associated with clinical stage and metastasis and can protect cells from staurosporine-induced apoptosis in an AKTdependent manner [221].

\section{The interplay of Nur77 and CHD1L}

Recent evidence demonstrates that Nur77 interacts directly with the CHD1L protein [229]. The C-terminal macro domain of CHD1L interacts with Nur77, inhibiting its nuclear-to-mitochondrial translocation and subsequent induction of apoptosis. CHD1L expression in a panel of HCC cell lines negatively correlates with induction of apoptosis following staurosporine treatment, further supporting CHD1L's role as an inhibitor of apoptosis and a potential mediator of drug resistance. It remains to be determined which residues of Nur77 are critical in the interaction with CHD1L and whether this interaction prevents binding of other proteins, such as RXR, or specific post-translational modifications of Nur77. Additionally, CHD1L is involved in chromatin remodeling and DNA repair $[217,218]$, which is mediated by its C-terminal macro domain through interactions with $\mathrm{Ku} 70$ and DNA-dependent protein kinase, catalytic subunit (DNA- 
PKcs). Given that Nur77 interacts with Ku80 to suppress DNA repair [200], it is plausible that a Nur77-CHD1L interaction could also repress chromatin remodeling and subsequent DNA repair, making the interaction mutually inhibitory. Also of interest is that CHD1L-mediated expression of SPOCK1 can activate AKT to maintain mitochondrial membrane potential, which prevents cytochrome $c$ release and apoptosis, all of which is blocked by pretreatment with an AKT1 inhibitor [221]. This scenario raises the possibility that CHD1L might inhibit Nur77 translocation through both a direct protein-protein interaction and through activation of AKT, which is known to be inhibitory to Nur77's mitochondrial association [137]. The regulation of Nur77 by CHD1L might offer a useful therapeutic avenue in which a small molecule could be developed to disrupt this interaction, allowing Nur77 to become fully functional in the cell death program.

\section{Concluding Remarks}

In summary, the NR4A family, represented by three highly homologous orphan receptors, plays multiple roles in cancer, with most studies highlighting the prooncogenic functions of Nur77 and Nurr1. In addition, Nur77 and Nor-1 have been characterized as being tumor suppressors in AML, likely due to their regulation of apoptosis in hematopoietic cells. This finding, in combination with other confounding results, as indicated by overexpression or downregulation in cancer cell lines and patient samples, shows the need to determine the cellular context in which the NR4A receptors contribute to oncogenesis or tumor suppression. It appears that multiple nuances can determine the role of NR4As in cancer, including but not limited to cell and tissue type, subcellular localization, external stimuli, protein-protein interactions, and posttranslational modifications.

The NR4A family is intertwined with many relevant cancer signaling pathways, which likely explains the dysregulated expression of these NRs in cancer, as well as their functions in tumorigenic hallmarks, including proliferation and survival (Figure 2-2). As an emerging research topic, it is highly likely that microRNAs are able to regulate the expression of NR4As in cancer, making their regulation and function much more interesting yet complex. Determining the contributions of microRNAs to NR4A regulation would provide further insight into NR4A dysregulation, as it is likely that a deleted or silenced tumor suppressor-like microRNA could target NR4A, explaining the general consensus of NR4A overexpression. Restoration of microRNA expression by using chemically modified and/or lipid-encapsulated mimics could lead to suppressed NR4A expression, ultimately reducing proliferation and inducing apoptosis.

Lastly, due to its dual functions in cell proliferation and death, Nur77 remains a unique drug target that several groups are targeting using small molecule approaches. One promising approach using small molecules would be to target the Nur77-CHD1L interface in hepatocellular carcinoma with 1q21 amplification to release nuclear-retained Nur77 from CHD1L; presumably, this type of small molecule could be used in 


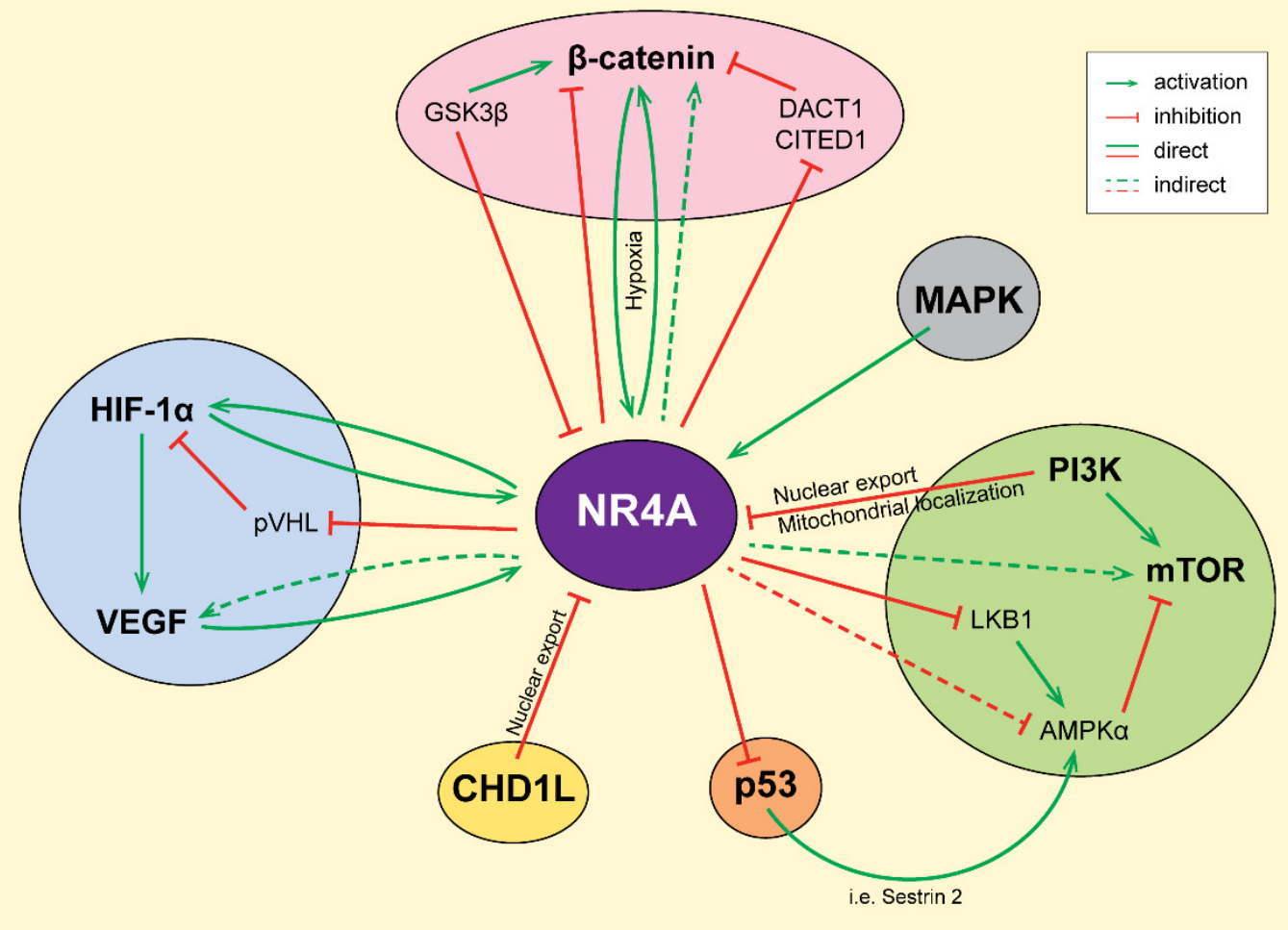

Figure 2-2. The NR4A family and key signaling pathways regulate each other. Both the expression and function of the NR4A members are mediated by activation or inhibition of multiple signaling pathways. Growth factor signaling upregulates the expression and nuclear localization of NR4A members and inhibits NR4A nuclear export and cell death. Additionally, NR4A members can either positively regulate oncogenic signaling pathways (i.e., HIF, $\beta$-catenin, mTOR) or overcome tumor suppressor signaling (i.e., pVHL, p53, LKB1). 
combination with compounds that induce Nur77 nuclear export to yield a higher apoptotic response. 


\section{CHAPTER 3. THE ORPHAN NUCLEAR RECEPTOR NR4A2 IS PART OF A P53-MICRORNA-34 NETWORK}

\section{Introduction}

The nuclear receptor (NR) superfamily is a group of ligand-regulated transcription factors that control specific gene activity. NRs are thus important drug targets [9, 15]. The 48 members of the human NR family share a common modular structure that includes an N-terminal domain containing an activation function 1 (AF-1) region, a DNA-binding domain (DBD), a hinge region, and a C-terminal ligand-binding domain (LBD) that also harbors an AF-2 domain. NR subfamily 4 group A (NR4A) consists of three highly homologous NRs, including NR4A2 (also called Nurr1, NOT, TINUR, or NGFI-B $\beta$ ), that are characterized as immediate-early genes induced by mitogens, growth factors, and other stimuli [78]. The NR4A receptors have been implicated as having roles in multiple tissues and diseases, including cancer [86], and their expression and function are associated with various oncogene and tumor suppressor pathways [45].

NR4A2 is involved in cancer progression through a mechanism that has yet to be fully described. Most research indicates that NR4A2 has an oncogenic-like role, as it mediates cell proliferation, survival, transformation, invasion, and migration $[88,90,92$, $118,209]$. NR4A2 is highly expressed in squamous cell carcinoma (SCC), as compared to normal patient tissues, and prostaglandin-mediated induction of NR4A2 expression in SCC leads to increased resistance to 5-fluorouracil (5-FU) [93]. The effects of NR4A2 on chemoresistance are also seen in colorectal cancers [94, 230], and high expression of NR4A2 predicts a poor outcome for gastric cancer patients receiving 5-FU therapy [231]. NR4A2 may mediate these effects through a previously described NR4A2-p53 interaction that serves to suppress p53 transactivation, thereby protecting cells from p53induced apoptosis [95]. Another NR4A family member, NR4A1 (also called Nur77, TR3, or NGFI-B $\alpha$ ), has also been implicated in p53 suppression [150].

As the main arbiter of cell cycle progression, DNA repair, and apoptosis, p53 is a central hub for regulating tumor suppression. These effects are mediated through $\mathrm{p} 53$ target genes, which include the microRNA-34 (miR-34) family [70, 71, 75, 232].

MicroRNAs (miRNAs) are small, endogenous, noncoding RNAs that help regulate target gene networks by binding complementarily to the 3 ' untranslated regions (UTRs) of target genes to degrade or prevent their translation into proteins [67]. The miR-34 family, which consists of three isoforms (miR-34a, miR-34b, and miR-34c) encoded by two p53 direct transcriptional target genes (mir-34a and mir-34b/c), is considered to be partly responsible for carrying out $\mathrm{p} 53$ 's tumor suppressive function by targeting the $3^{\prime}$ UTRs of genes that are critical to the cell cycle and survival, such as BCL2, cyclins, cyclindependent kinases (CDKs), and $M Y C N$ [75, 233]. In mice, mir-34a is expressed at higher levels than mir-34b/c, except in lung tissues, where mir-34b/c is dominantly expressed [75]. Although miR-34 is dispensable for p53 tumor suppression [234], it is critical for enhancing p53 stability and activity through miR-34-mediated suppression of negative regulators of p53, such as Sirt1 [235] and Hdm4 [236]. The miR-34 isoforms have been 
clinically characterized as tumor suppressors in multiple cancer types, often independent of p53 mutation, as in neuroblastoma, where mir-34a is commonly deleted [73, 74], or in other cancers characterized by epigenetic silencing of miR-34 [237, 238].

Recently, miR-132 was reported to target mouse $\operatorname{Nr} 4 a 2$ [239, 240]; the first characterization of an miRNA targeting an NR4A member. In this study, to determine whether human NR4A2 is regulated by miRNAs and investigate its oncogenic-like role, we used an miRNA screening approach to identify cancer-relevant posttranscriptional regulatory networks of $N R 4 A 2$, a subject that has not been fully explored. We identified miR-34 as a direct regulator of NR4A2 through a specific sequence in its $3^{\prime}$ UTR. Furthermore, we determined that elevated miR-34 levels, resulting from exogenous overexpression or endogenous induction in a p53-dependent manner, decreased the levels of NR4A2. Corroborating the reported NR4A2-mediated inhibition of p53 [95], we found that overexpression of NR4A2 inhibited the ability of p53 to activate its target genes, including mir-34a. Lastly, overexpression of NR4A2 attenuated the sensitivity of cells to the p53 activator Nutlin-3a. These data are consistent with previous findings and, for the first time, identify miR-34 as a direct negative regulator of $N R 4 A 2$. Together, they reveal a novel regulatory network linking p53, miR-34, and NR4A2, in which p53 can overcome its inhibition by endogenous NR4A2 through upregulating miR-34.

\section{Results}

\section{Identification of miRNAs directly targeting the $3^{\prime}$ UTR of $N R 4 A 2$}

Increased $N R 4 A 2$ expression in cancer has been characterized as resulting from cell signaling events [45], but miRNA-mediated regulation of NR4A2 in cancer has hitherto been unexplored. To identify putative miRNAs capable of regulating $N R 4 A 2$ through its 3' UTR, we assessed the effects of 75 cancer-relevant miRNAs by using a luciferase reporter system (Figure 3-1a). Precursor miRNAs selected from an established library [241] (Table A-1) or an miRNA negative control (pSIF) were cotransfected with the NR4A2 3' UTR reporter plasmid (WT 3UTR) in 293T cells. We observed that several miRNAs, including miR-34c, were capable of decreasing the luminescence reporter signal to a significantly greater extent than was miR-132, an miRNA that targets $\mathrm{Nr} 4 \mathrm{a} 2$ $[239,240]$ ( $\log 2$ fold changes of -0.47521 and -0.29685 , respectively; $P \leq 0.0001$ and $P=0.0086$, respectively) (Figure 3-1b and Table 3-1). This suggested that miR-34c was a modulator of $N R 4 A 2$ through its $3^{\prime}$ UTR.

By using three different miRNA prediction algorithm tools (TargetScan [242], miRanda [243], and PicTar [244]), we identified NR4A2 as a predicted target of the miR34 family (Table 3-1), further suggesting that this family of miRNAs were regulators of $N R 4 A 2$. As expected, miR-132 was also predicted to target NR4A2. To corroborate the findings from "first-generation" prediction tools, we used the CoMeTa interactive database [245], which integrates thousands of publicly available gene-expression datasets with the assumption that the predicted targets of an miRNA will be coexpressed with 
a.

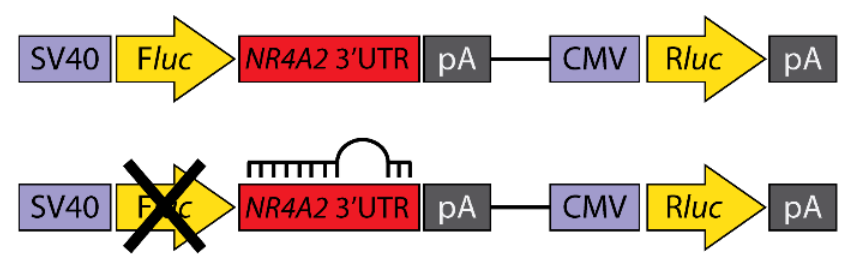

C.

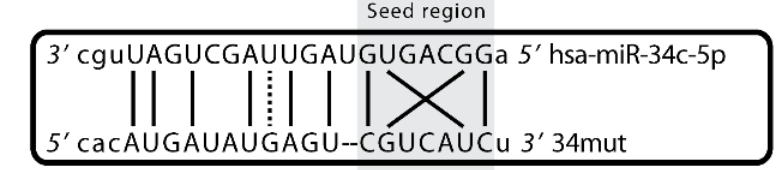

5' cacAUGAUAUGAGU--CACUGCCU 3' WT 3UTR

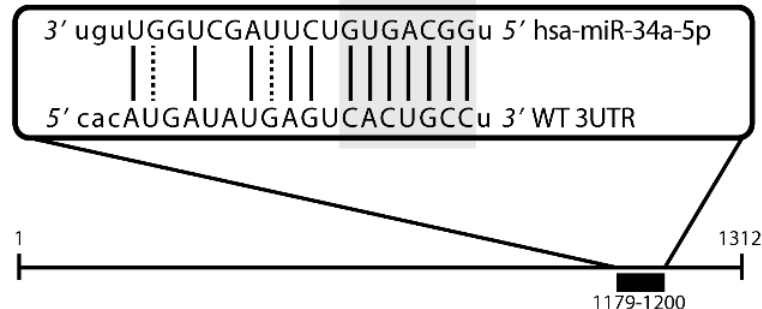

b.

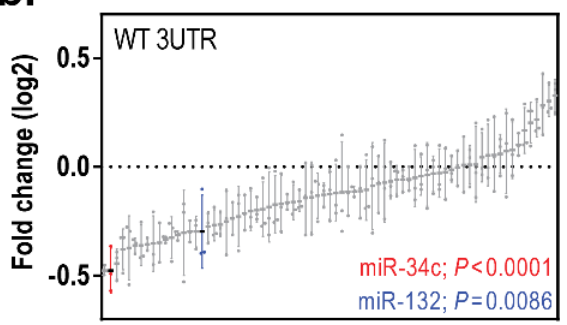

d.

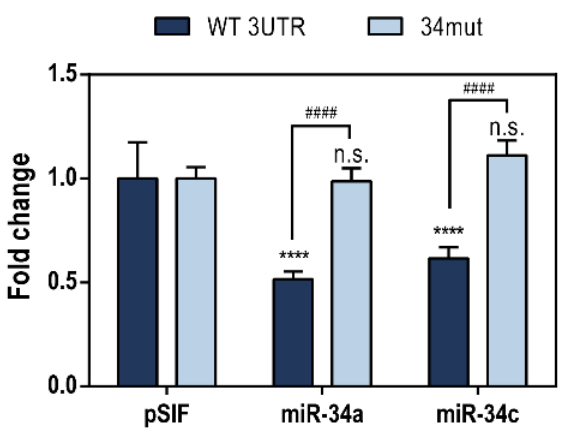

Figure 3-1. Screening for miRNAs that directly target the $3^{\prime}$ UTR of $N R 4 A 2$. (a) A reporter plasmid containing the $3^{\prime}$ UTR of $N R 4 A 2$ downstream from a firefly luciferase $(\mathrm{Fluc})$ gene was used to identify miRNAs that putatively regulate $N R 4 A 2$, relative to an internal Renilla luciferase (Rluc) control gene. (b) $293 \mathrm{~T}$ cells were transfected with the NR4A2 3' UTR (WT 3UTR) reporter construct for $24 \mathrm{~h}$. Transfected cells were reseeded in 96-well plates and reverse transfected with 75 individual cancerrelevant miRNAs. After $48 \mathrm{~h}$ of transfection, a Dual-Glo luciferase assay was performed, the ratio of Fluc/Rluc was calculated, and the log2 fold change was determined for each miRNA $(n=3)$, relative to a transfection control (pSIF, $n=9)$, and presented as a waterfall plot. The Fluc/Rluc value for pSIF was set as 1. Statistical significance was calculated using a one-way ANOVA and Dunnett's test for multiple comparisons between pSIF and the indicated miRNAs. (c) A schematic of the predicted miR-34 seed region in the NR4A2 $3^{\prime}$ UTR (WT 3UTR). This predicted miR-34 binding site in the NR4A2 3' UTR was mutated, and the resulting loss of complementarity is shown (34mut). (d) WT 3UTR or 34mut reporter constructs were cotransfected with pSIF or the indicated miR-34 isoforms into HCT116 colorectal carcinoma cells for $72 \mathrm{~h}$, after which a DualGlo luciferase assay was performed. The fold change of the Fluc/Rluc ratio with respect to $\mathrm{pSIF}$ was calculated (the Fluc/Rluc value of pSIF for each reporter transfection group was set as 1), and the statistical significance of the relation between pSIF and miR-34a or miR-34c was determined using a two-way ANOVA and Tukey's test for multiple comparisons (****, $P \leq 0.0001$; n.s., $P>0.05)$. Statistically significant changes in WT 3UTR and 34mut for each miRNA transfection are indicated by \#\#\#\# $(P \leq 0.0001)$. 
Table 3-1. MicroRNAs that putatively regulate $N R 4 A 2$ through its $3^{\prime}$ UTR.

\begin{tabular}{|c|c|c|c|c|c|c|c|c|}
\hline Rank & miRNA ID & $\begin{array}{l}\text { Log2 fold } \\
\text { change }\end{array}$ & $P$-value & TargetScan & miRanda & PicTar & $\begin{array}{l}\text { Other predicted } \\
N R 4 A \text { genes }\end{array}$ & Other targets \\
\hline 1 & hsa-miR-335 & $\begin{array}{l}-0.47549 \\
\pm 0.0223\end{array}$ & $<0.0001$ & No & No & No & NR4A3 & $\begin{array}{c}\text { BCL2L2, SOX4, RB1, } \\
\text { RUNX2 }\end{array}$ \\
\hline 2 & hsa-miR-34c & $\begin{array}{l}-0.4752 \\
\pm 0.1028\end{array}$ & $<0.0001$ & Yes & Yes & Yes & --- & $\begin{array}{c}\text { BCL2, CCND1, CDK4/6, } \\
\text { FRA1, MET, MYC, MYCN } \\
\text { SIRT1, SNAI1 }\end{array}$ \\
\hline 3 & hsa-miR-144 & $\begin{array}{l}-0.44443 \\
\pm 0.0696\end{array}$ & $<0.0001$ & No & No & No & NR4A3 & NOTCH1, PTEN, TGFB1 \\
\hline 4 & hsa-miR-214 & $\begin{array}{l}-0.37908 \\
\pm 0.0942\end{array}$ & 0.0002 & No & Yes & Yes & NR4A1 & $\begin{array}{c}\text { BCL2L2, EZH2, PTEN, } \\
\text { TWIST1 }\end{array}$ \\
\hline 5 & hsa-miR-191 & $\begin{array}{l}-0.37123 \\
\pm 0.1471\end{array}$ & 0.0002 & No & No & No & --- & CDK6, SOX4 \\
\hline 6 & hsa-miR-15a & $\begin{array}{l}-0.36165 \\
\pm 0.0343\end{array}$ & 0.0004 & No & No & No & NR4A1, NR4A3 & $\begin{array}{l}\text { BCL2, CCND1, CCND2, } \\
\text { CCNE1, CRKL, VEGFA }\end{array}$ \\
\hline 7 & hsa-miR-155 & $\begin{array}{l}-0.36052 \\
\pm 0.0926\end{array}$ & 0.0004 & No & No & No & NR4A3 & $\begin{array}{c}A P C, F O X O 3, M L H 1, \\
R U N X 2, S M A D 1, S M A D 2, \\
\text { SMAD5 }\end{array}$ \\
\hline 8 & hsa-miR-20a & $\begin{array}{l}-0.35512 \\
\pm 0.0296\end{array}$ & 0.0005 & Yes & Yes & Yes & NR4A3 & $\begin{array}{c}C C N D 1, C D K N 1 A, E 2 F 1, \\
\text { HIF1A, KIT, PTEN }\end{array}$ \\
\hline 9 & hsa-miR-25 & $\begin{array}{l}-0.35227 \\
\pm 0.128\end{array}$ & 0.0006 & No & No & No & NR4A3 & $\begin{array}{c}\text { BCL2L11, CDH1, EZH2, } \\
M D M 2, T P 53\end{array}$ \\
\hline 17 & hsa-miR-132 & $\begin{array}{c}-0.296850 \\
\pm 0.1684 \\
\end{array}$ & 0.0086 & Yes & Yes & Yes & --- & $\begin{array}{c}C D K N 1 A, N R 4 A 2, R B 1, \\
\text { SIRT1 }\end{array}$ \\
\hline
\end{tabular}


each other. The authors noted that targets falling within the top $50^{\text {th }}$ percentile of the CoMeTa analysis "co-rank" list for putative miRNA targets are highly predictive, based on previous dataset validation. CoMeTa analysis indicates that NR4A2 falls within the top 40.568 and 39.182 percentiles for miR-34a and miR-34c-5p, respectively. By using the prediction algorithms, we identified a predicted miRNA recognition element complementary to the miR-34 seed region in the 3' UTR of NR4A2 and mutated this sequence to disrupt the complementarity in order to determine if there was direct regulation by miR-34 at this specific site (Figure 3-1c). The wild-type 3' UTR (WT 3UTR) reporter signal was effectively suppressed by cotransfection of miR-34a or miR$34 \mathrm{c}$ (52\% and $62 \%$ of the control level, respectively; $P \leq 0.0001$ for all) (Figure 3-1d). The effect of miR-34a or miR-34c, compared to that of the control, was not significant when the miRNA was cotransfected with the $3^{\prime}$ UTR containing the seed-region mutation (34mut). Mutation of this predicted seed region was able to rescue the attenuation of the luminescence signal by miR-34a or miR-34c (Figure 3-1d), increasing the signal from $52 \%$ to $99 \%$ and from $62 \%$ to $111 \%$ of the control level, respectively ( $P \leq 0.0001$ for all). These data indicate that miR-34 can target $N R 4 A 2$ at a specific seed complementarity region within its 3' UTR. Furthermore, by analyzing a dataset from 97 patient samples with rectum adenocarcinoma, we found that the expression of $N R 4 A 2$ and miR-34a was inversely correlated to a similar extent as other miR-34 targets $A X L$ [246] and SIRT1 [235] (Figures A-1 and A-2). The read counts for miR-34b and miR-34c in this dataset were very low (data not shown), which is consistent with the known expression patterns of mir-34b/c primarily in lung tissues[75], making correlation analyses for the $\mathrm{mir}-34 \mathrm{~b} / \mathrm{c}$ isoforms not suitable.

\section{miR-34 regulates endogenous $N R 4 A 2$ levels}

We next determined the in vitro effect of miR-34 on endogenous NR4A2 mRNA and protein levels through the use of miRNA mimics. Mature miR-34a-5p or miR-34c-5p mimics or a control (CmiR) were overexpressed in HCT116 ${ }^{T P 53-/-}(\mathrm{KO})$ or HCT116 wild-type (WT) isogenic cell lines to assess the direct effects of forced miRNA expression on NR4A2. Transfection of mature miR-34 mimics increased the expression of each miRNA (Figure 3-2a, b). Transfection of miR-34a-5p slightly increased miR-34c$5 p$ expression, probably because of specificity differences in the stem loop primers caused by the approximately $80 \%$ sequence homology of the two miR-34 isoforms. Overexpression of the miR-34a-5p mimic reduced the endogenous $N R 4 A 2$ expression to $59 \%$ and $64 \%$ of the control level in KO and WT cells, respectively, by $12 \mathrm{~h}$ posttransfection, whereas overexpression of the miR-34c-5p mimic reduced the NR4A2 expression to $71 \%$ and $66 \%$ of the control level in KO and WT cells, respectively (Figure 3-2c), and this effect was independent of p53 status. At $24 \mathrm{~h}$ post-transfection, the effect of the miR-34 mimics on NR4A2 levels in HCT116 with wild-type p53 was no longer obvious, by comparison to the control. Additionally, the transfection of miR-34 mimics led to increased expression of the p53 target gene $C D K N 1 A / \mathrm{p} 21$ (Figure 3-2d), increased p53 protein expression and acetylation, and subsequent p 21 protein levels (Figure A-3) in wild-type HCT116 cells. The observation that increased levels of miR-34 
a.

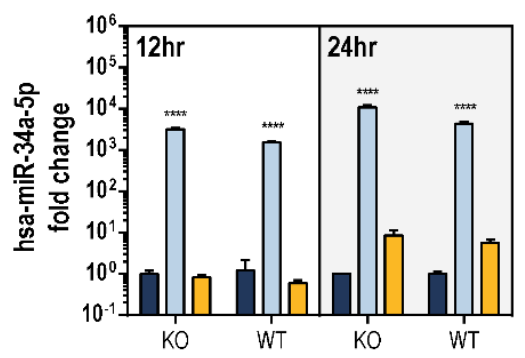

c.

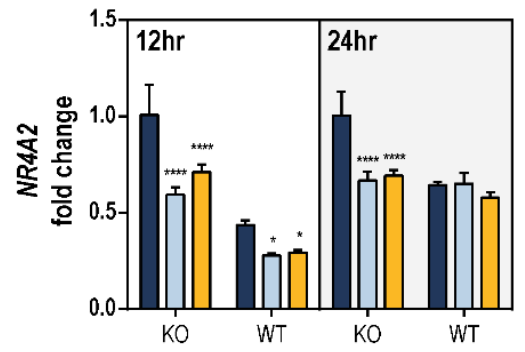

b.
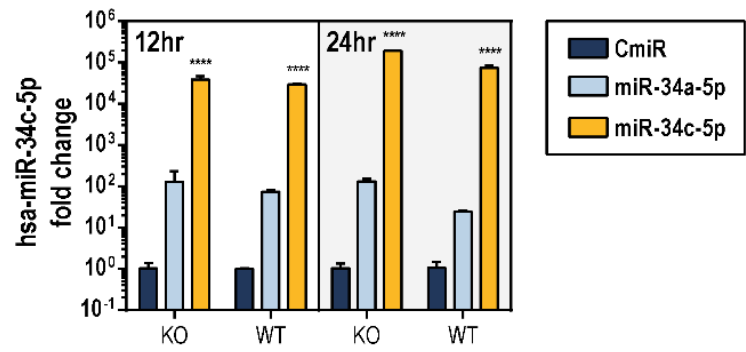

d.

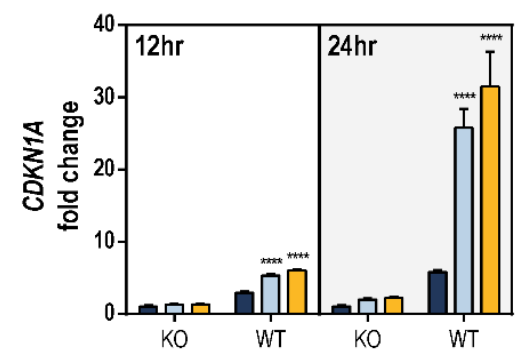

e.

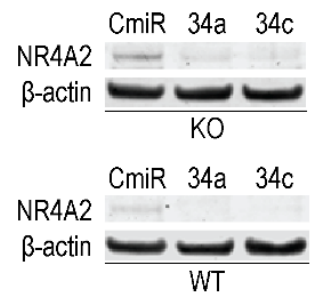

Figure 3-2. miRNA-34 downregulates endogenous NR4A2.

Mature miR-34a and miR-34c mimics $(10 \mathrm{nM})$ were transfected into HCT116 ${ }^{\text {TP53-/- }}$ (KO) or HCT116 wild-type (WT) cells for 12 or $24 \mathrm{~h}$. Expression of hsa-miR-34a-5p (a), hsa-miR-34c-5p (b), NR4A2 (c), and $C D K N 1 A$ (d) was determined using TaqMan qPCR probes. Mature miRNA expression was normalized to $R N U 6 B$, and $N R 4 A 2$ and $C D K N 1 A$ were normalized to $G A P D H$. The value for the CmiR-transfected $\mathrm{KO}$ cells at each time point was set as 1 . The statistical significance of the results of miR-34 transfections, compared to those of the control, in each cell line for each time point was calculated using a two-way ANOVA and Dunnett's test for multiple comparisons. ${ }^{* * * *}, P \leq 0.0001$; $*, P \leq 0.05$. (e) Whole-cell lysates $(45 \mu \mathrm{g})$ from HCT116 ${ }^{\text {TP53-/- }}(\mathrm{KO})$ and HCT116 wildtype (WT) cells transfected for $48 \mathrm{~h}$ with control (CmiR), miR-34a (34a), or miR-34c (34c) mimics $(10 \mathrm{nM})$ were assessed for expression of NR4A2 and $\beta$-actin protein by performing Western blotting analysis. 
led to enhanced p53 protein expression and activity probably reflects the known suppressive function of miR-34 on negative regulators of $\mathrm{p} 53$ protein stability (Figure A-3) [235, 236], though TP53 gene expression (in terms of mRNA levels) was also slightly increased by the miR-34 mimics (Figure A-3). The slight but significant increase in TP53 expression induced by the miR-34 mimics is consistent with the observation that the levels of NR4A2 correlate inversely with that of TP53 gene expression, as discussed later. After $48 \mathrm{~h}$ of miR-34 mimic overexpression in HCT116 cells, the protein levels of NR4A2 (Figure 3-2e) and another miR-34 target, Sirt1 (Figure A-3), were reduced in both KO and WT cells. Additionally, the effect of mature miR-34 mimics on NR4A2 expression was assessed in another colorectal cancer cell line, RKO, with similar results observed on $C D K N 1 A$ and $N R 4 A 2$ expression (Figure A-4). These data demonstrate that exogenous overexpression of a mature miR-34 decreases the levels of endogenous NR4A2 at both mRNA and protein level, regardless of the p53 status. The differential effect of exogenous miR-34 mimic on endogenous NR4A2 may be attributable to the different levels of endogenous $N R 4 A 2 \mathrm{mRNA}$ in TP53+/+ and TP53-/- cells, although NR4A2 protein expression was substantially decreased in both TP53-isogenic backgrounds in response to exogenous miR-34 mimic.

\section{p53 activation suppresses endogenous $N R 4 A 2$ levels}

As mir-34a is a direct transcriptional target of p53 [71,232], we next sought to determine if $N R 4 A 2$ expression was regulated by endogenous miR-34a in a $\mathrm{p} 53-$ dependent manner. We treated cells with a chemical activator of p53, Nutlin-3a [247], and confirmed that the p53 protein level increased after the treatment in cells expressing wild-type p53 (WT) but not in p53-deficient (KO) cells (Figure 3-3a). As expected, the expression of transcriptional targets of $\mathrm{p} 53, C D K N 1 A / \mathrm{p} 21$ and $B B C 3 / \mathrm{Puma}$, was also induced in a p53-dependent manner (Figure 3-3a). HCT116 ${ }^{C D K N 1 A-/-}$ and HCT116 ${ }^{B B C 3-/-}$ cells were used to demonstrate that Nutlin-3a acts upstream of $C D K N 1 A / \mathrm{p} 21$ and $B B C 3$ Puma through p53 (Figure 3-3a). We also found increased expression of $C D K N 1 A / \mathrm{p} 21$ and mir-34a in cells expressing wild-type p53 (Figure 3-3b, c), accompanied by decreased $N R 4 A 2$ transcript levels after $48 \mathrm{~h}$ of treatment with Nutlin-3a (Figure 3-3d). The NR4A family, including $N R 4 A 2$, are immediate-early responsive genes that are inducible by many external stimuli [78], and at an earlier time point $(24 \mathrm{~h})$, the levels of NR4A2 mRNA were actually increased by Nutlin-3a (Figure 3-3d). In agreement with mRNA expression, Nutlin-3a treatment for $48 \mathrm{~h}$ also caused a decrease in the NR4A2 protein expression in WT cells, but not in KO cells (Figure 3-3e), further demonstrating the p53 requirement for Nutlin-3a-induced NR4A2 decrease. In addition to NR4A2, Nutlin-3a was also capable of decreasing the protein expression of Sirt1 (Figure 3-3e), a known target of miR-34 [235], suggesting that p53 activation can regulate other downstream factors, possibly through miR-34. We confirmed these results in other colorectal cell lines (RKO and SW48) that are isogenic for TP53

(Figures A-5 and A-6). These data indicate that p53 activation can induce the expression of mir-34a, while also decreasing the endogenous levels of NR4A2 and another known 
a.
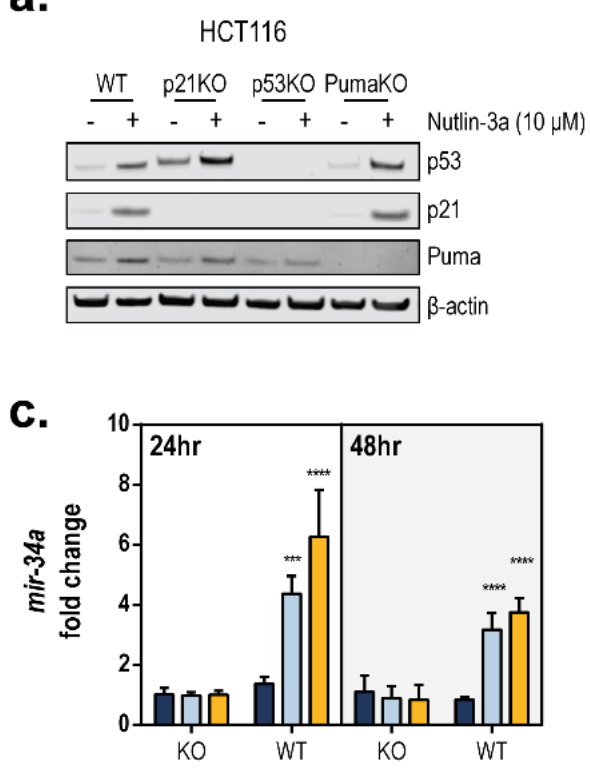

b.
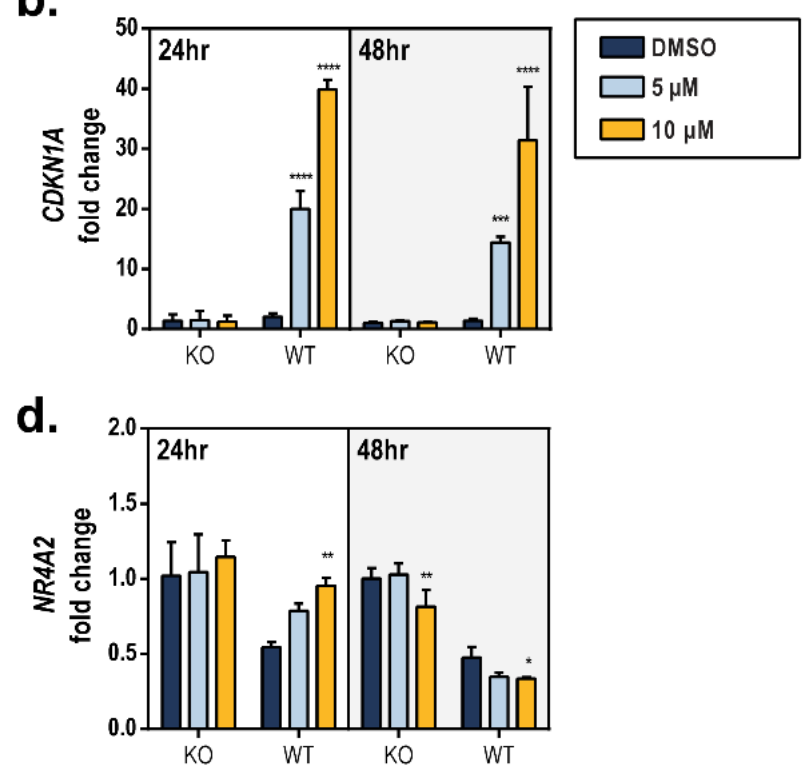

e.

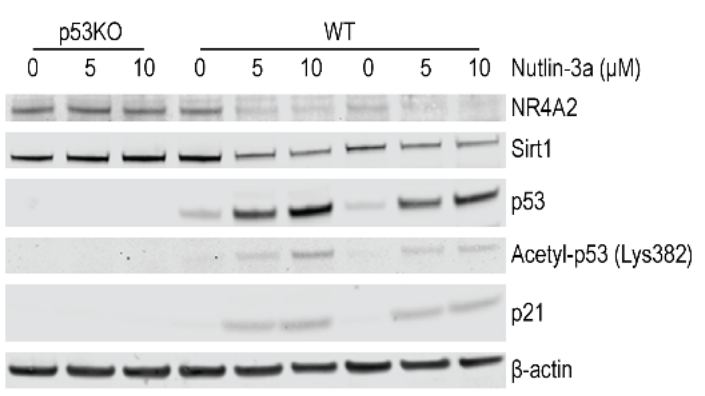

Figure 3-3. Nutlin-3a activation of p53 decreases endogenous NR4A2.

(a) HCT116 wild-type (WT), HCT116 ${ }^{\text {CDKN1A-/- }}$ (p21KO), HCT116 ${ }^{\text {TP53--- }}$ (p53KO), and HCT116 ${ }^{B B C 3-/-}$ (PumaKO) isogenic cell lines were treated for $48 \mathrm{~h}$ with $10 \mu \mathrm{M}$ of Nutlin-3a or vehicle control (DMSO). Whole-cell lysates $(40 \mu \mathrm{g})$ were assessed for expression of p53, p21, Puma, and $\beta$-actin by SDS-PAGE gel electrophoresis. (b-d) HCT116 ${ }^{T P 53--}(\mathrm{KO})$ and HCT116 wild-type (WT) cells were treated with vehicle control (DMSO) or Nutlin-3a (5 or $10 \mu \mathrm{M})$ for 24 or $48 \mathrm{~h}$. Expression of CDKN1A (b), mir-34a (c), and NR4A2 (d) was determined using TaqMan qPCR probes (normalized to $G A P D H)$. The value for DMSO-treated KO cells at each time point was set as 1 . The statistical significance of the results obtained with Nutlin-3a treatments, compared to those obtained with DMSO, in each cell line for each time point was calculated using a two-way ANOVA and Dunnett's test for multiple comparisons. ****, $P \leq 0.0001$; ***, $P \leq 0.001$; ** $P \leq 0.01$; $^{*}, P \leq 0.05$. (e) Whole-cell lysates $(45 \mu \mathrm{g})$ from HCT116 ${ }^{\text {TP53- }-}$ (p53KO) and HCT116 wild-type (WT) cells treated for $48 \mathrm{~h}$ with DMSO $(0 \mu \mathrm{M})$ or Nutlin-3a $(5$ or $10 \mu \mathrm{M})$ were assessed for expression of indicated proteins by performing Western blotting analysis. 
target of miR-34 (Sirt1). These results are consistent with the observation that the levels of miR-34 and NR4A2 are inversely correlated.

\section{Overexpression of $N R 4 A 2$ suppresses p53 activation}

It was previously reported that NR4A2 suppressed the transcriptional activity of p53 in the presence or absence of doxorubicin [95]. We sought to determine the effects of NR4A2 overexpression on the induction of p53 activation by Nutlin-3a. HCT116 cells were transduced with lentivirus expressing empty vector control (EV) or 3xFlag-NR4A2, and overexpression of $N R 4 A 2$ was confirmed (Figure 3-4a). The EV- or 3xFlag-NR4A2transduced cells were treated with Nutlin-3a to determine the effect on p53 downstream target genes. Upon Nutlin-3a treatment, overexpression of $N R 4 A 2$ led to a significant attenuation of the p53-induced expression of the target genes mir-34a and CDKN1A/p21 (Figure 3-4b, c). To assess the effect of NR4A2 on the binding of p53 to target gene promoters, we performed a chromatin immunoprecipitation (ChIP) assay. As shown in Figure A-7, NR4A2 did not decrease p53 occupancy at the target gene promoters [248, 249], suggesting that another mechanism is responsible for the inhibitory effect of NR4A2 on p53. However, HCT116 cells overexpressing NR4A2 had diminished levels of p53 mRNA and protein (Figure 3-4d, e and Figure A-7). These data demonstrate that NR4A2 exerts inhibitory effects on the levels of p53 and its transcriptional target genes, which is consistent with previously reported results [95] and suggests that the attenuation of p53 mRNA and protein levels, but not of p53 binding to target gene promoters, probably contributes to such inhibitory effects.

\section{Knockdown of $N R 4 A 2$ enhances p53 activation}

To further confirm the inhibitory effect of NR4A2 on p53 transcriptional activity, we investigated the effects of reduced endogenous $N R 4 A 2$ levels on Nutlin-3a-induced p53 transcriptional target gene expression. HCT116 cells were transfected with nontargeting control (NT) or siRNA targeting NR4A2 (siNR4A2), and knockdown of $N R 4 A 2$ was confirmed (Figure 3-5a). After $48 \mathrm{~h}$ of siRNA-mediated knockdown, the cells were treated with Nutlin-3a for an additional $24 \mathrm{~h}$ to determine the effects of $N R 4 A 2$ knockdown on p53 target gene expression. We found that knocking down NR4A2 caused an enhancement of Nutlin-3a-induced expression of CDKN1A/p21, MDM2, and mir-34a (Figure 3-5b-d). Consistent with the inhibitory effect of overexpressed NR4A2 on p53 gene expression (Figure 3-4d), knocking down NR4A2 led to an increase in TP53/p53 gene expression for both the DMSO- and Nutlin-3a-treated groups (Figure 3-5e). Together, these data further suggest that NR4A2 can suppress p53 transcriptional activity, at least in part, by inhibiting the expression of p53 itself. 
a.

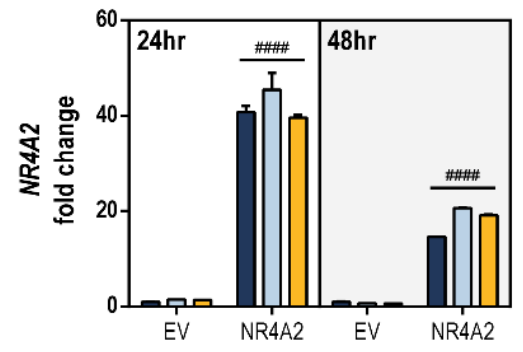

C.

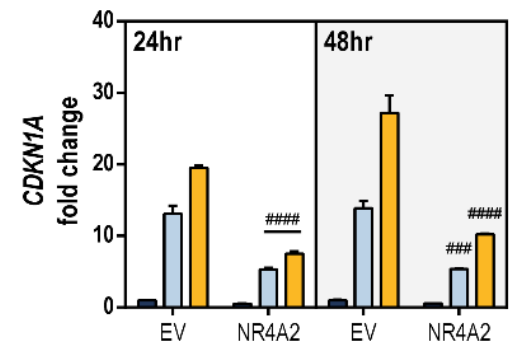

b.

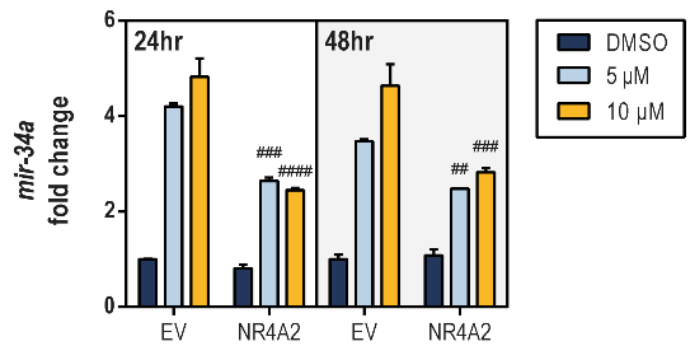

d.

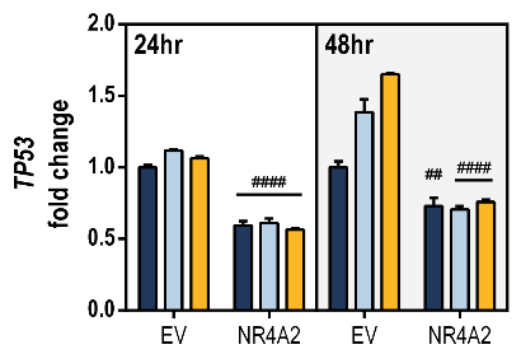

e.

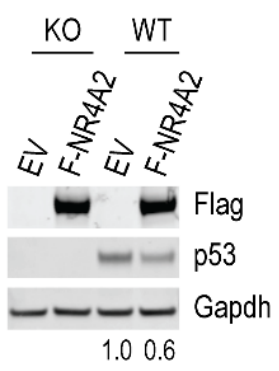

Figure 3-4. NR4A2 overexpression suppresses p53 activation.

HCT116 cells were transduced for $16 \mathrm{~h}$ overnight with lentivirus expressing empty vector (EV) or 3xFlag-NR4A2 (NR4A2). The cell medium was then changed and the cells remained in culture for a total of $48 \mathrm{~h}$. The cells were then reseeded and treated with vehicle (DMSO) or Nutlin-3a (5 or $10 \mu \mathrm{M}$ ) for 24 or $48 \mathrm{~h}$. Expression of NR4A2 (a), mir$34 a$ (b), CDKN1A (c), and TP53 (d) was determined using TaqMan qPCR probes (normalized to $G A P D H$ ). The value for DMSO-treated $\mathrm{EV}$ at each time point was set as 1. The statistical significance of the difference between the results with EV and NR4A2 for each treatment was determined for each time point by using a two-way ANOVA with Sidak's multiple comparisons test. \#\#\#\#, $P \leq 0.0001$; \#\#\#, $P \leq 0.001$; \#\#, $P \leq 0.01$; \#, $P \leq 0.05$. (e) Whole-cell lysates from HCT116 ${ }^{T P 53-/-}(\mathrm{KO})$ and HCT116 wild-type (WT) cells transduced with lentivirus expressing EV or 3xFlag-NR4A2 (F-NR4A2) were assessed for expression of Flag (indicating NR4A2), p53, and Gapdh protein by performing SDS-PAGE gel electrophoresis. Relative p53 expression was determined using Odyssey Image Studio to calculate the ratio of p53 to Gapdh protein band density (displayed at the bottom of the gel). The ratio for EV in WT cells was set as 1.0. 

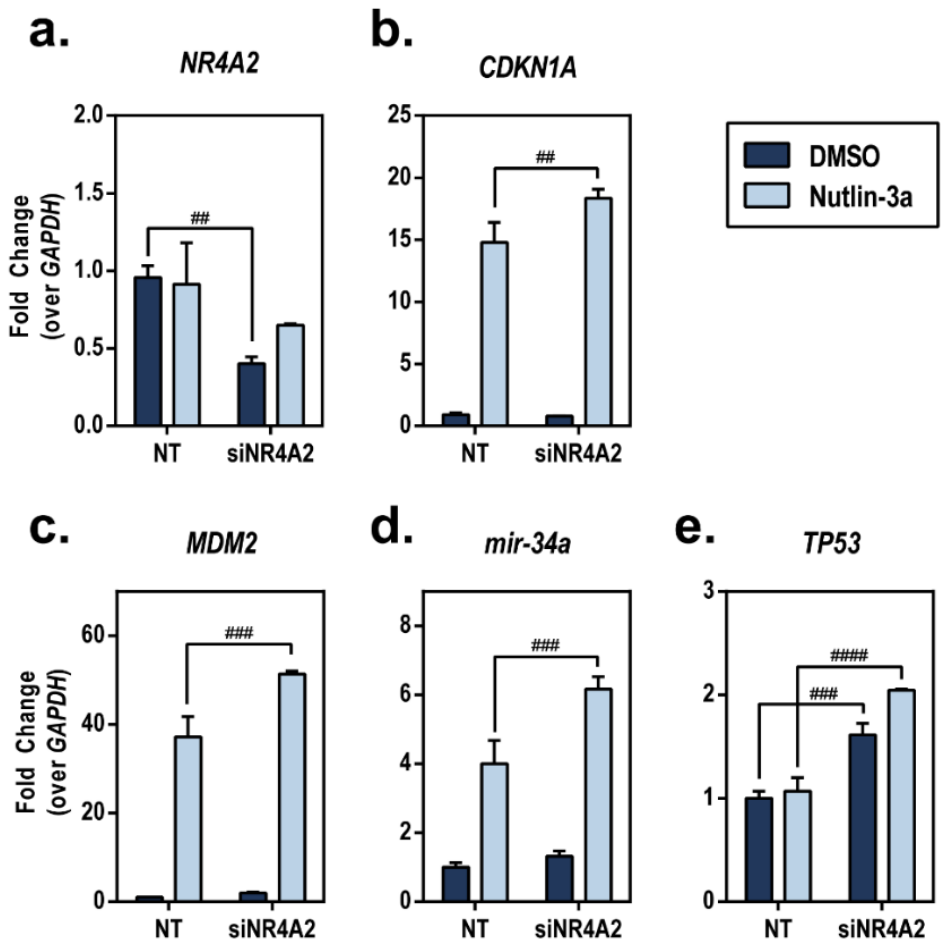

Figure 3-5. Knockdown of $N R 4 A 2$ enhances p53 activation.

HCT116 cells were transfected for $24 \mathrm{~h}$ with nontargeting control (NT) or siRNA targeting NR4A2 (siNR4A2). The culture medium was then changed, and the cells remained in culture for a total of $48 \mathrm{~h}$. The cells were then treated with vehicle (DMSO) or Nutlin-3a $(10 \mu \mathrm{M})$ for $24 \mathrm{~h}$. Expression of $N R 4 A 2$ (a), CDKN1A (b), MDM2 (c), mir$34 a$ (d), and TP53 (e) was determined using TaqMan qPCR probes (normalized to $G A P D H)$. The value for DMSO-treated NT was set as 1 . The statistical significance of the results was determined using a two-way ANOVA with Tukey's multiple comparison test. The significance of the differences within each transfection group between transfection groups (\#\#\#, $P \leq 0.0001$; \#\#\#, $P \leq 0.001$; \#\#, $P \leq 0.01$ ) is represented. 


\section{Overexpression of $N R 4 A 2$ attenuates Nutlin-3a sensitivity}

We next examined the cellular effects of NR4A2-mediated suppression of p53 after Nutlin-3a treatment. HCT116 cells transduced for $48 \mathrm{~h}$ with lentivirus expressing empty vector control (EV) or 3xFlag-NR4A2 were plated in normal growth medium, and the cell proliferation was monitored using live-cell imaging. After $14 \mathrm{~h}$, the cell culture medium was replaced with fresh medium containing vehicle (DMSO) or Nutlin-3a (5 or $10 \mu \mathrm{M}$ ), and the monitoring of cell proliferation continued. In EV-expressing cells, the inhibitory effect of Nutlin-3a on cell proliferation was substantial (Figure 3-6a). In cells overexpressing $N R 4 A 2$, Nutlin-3a-induced inhibition of cell proliferation was attenuated at both concentrations of Nutlin-3a tested (Figure 3-6b). At the end of the real-time monitoring period $(108 \mathrm{~h})$, the cells were collected and their gene expression was examined to confirm that $N R 4 A 2$ expression was increased in cells transduced with $N R 4 A 2$-expressing lentivirus (Figure 3-6c). These data indicate that overexpression of $N R 4 A 2$ attenuates the inhibitory effect of Nutlin-3a on cell proliferation. Together, our data are consistent with the known oncogenic role of $N R 4 A 2$ and, for the first time, identify miR-34 as a negative regulator of $N R 4 A 2$ and reveal a novel functional network linking p53, miR-34, and p53.

\section{Discussion}

Over the past decade, it has become increasingly evident that miRNA dysregulation plays an important role in human disease, including the development, progression, and therapeutic resistance of cancer. This process can be quite complex, as overlapping miRNA-mRNA networks can be formed, with a single miRNA having multiple targets or a single mRNA being a target for multiple miRNAs [250]. Research on miRNA regulation and the validation of biological targets has continued to increase in an effort to understand the multiple cellular pathways that are affected by specific miRNAs, and this area remains of particular interest as additional miRNA-based therapies are investigated and placed into clinical trials [251, 252].

The NR4A family of orphan nuclear receptors has been studied extensively in various cancer models, and its regulation and function have been connected to multiple oncogenic and tumor suppressive pathways [45]. However, the contribution of miRNAs to the expression of the NR4A family is unclear. In this report, we have presented a novel p53-miR-34 regulatory mechanism of the orphan nuclear receptor NR4A2, in which miR-34 directly and negatively regulates NR4A2, which is itself able to repress p53induced gene expression to rescue the inhibition of cell proliferation (Figure 3-7). This is the first miRNA to be characterized as targeting a member of the human NR4A family $[239,240]$.

Based on the known roles of NR4A2 in oncogenic processes within cancer, we posited that a likely candidate miRNA would be one that harbored tumor-suppressive functions and was downregulated in cancer, consequently leading to upregulation of NR4A2. As presented here, we identified and described the direct regulation of NR4A2 
a.

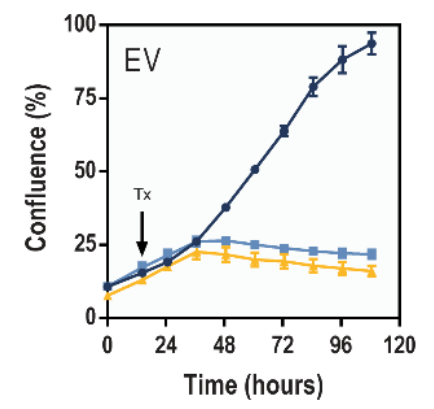

b.

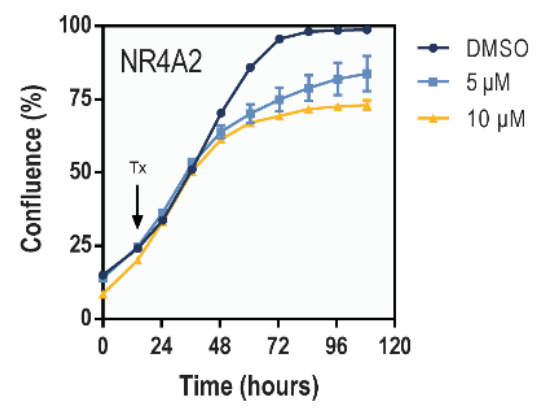

C.

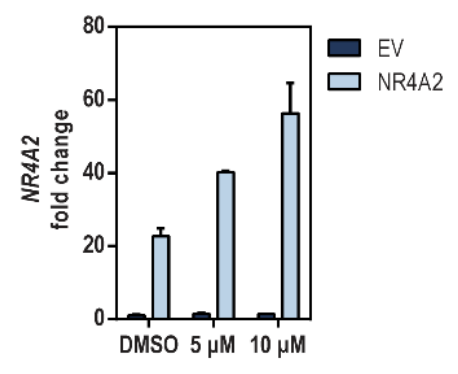

Figure 3-6. Overexpression of $N R 4 A 2$ attenuates Nutlin-3a sensitivity.

HCT116 cells were transduced for $16 \mathrm{~h}$ overnight with lentivirus expressing empty vector (EV) or 3xFlag-NR4A2 (NR4A2). The cell medium was then changed, and the cells remained in culture for a total of $48 \mathrm{~h}$. The cells were then reseeded into plates and the cell confluence was monitored using an IncuCyte ZOOM imaging system. After $14 \mathrm{~h}$, the cell medium was replaced by medium containing vehicle (DMSO) or Nutlin-3a (5 or 10 $\mu \mathrm{M}$ ) (indicated by Tx and arrow). The cell confluence for EV-expressing (a) or NR4A2expressing (b) cells was monitored for a total of $108 \mathrm{~h}$. (c) The cells were then collected and expression of $N R 4 A 2$ was determined using TaqMan qPCR probes (normalized to $A C T B)$. The value for DMSO-treated EV was set as 1 . 


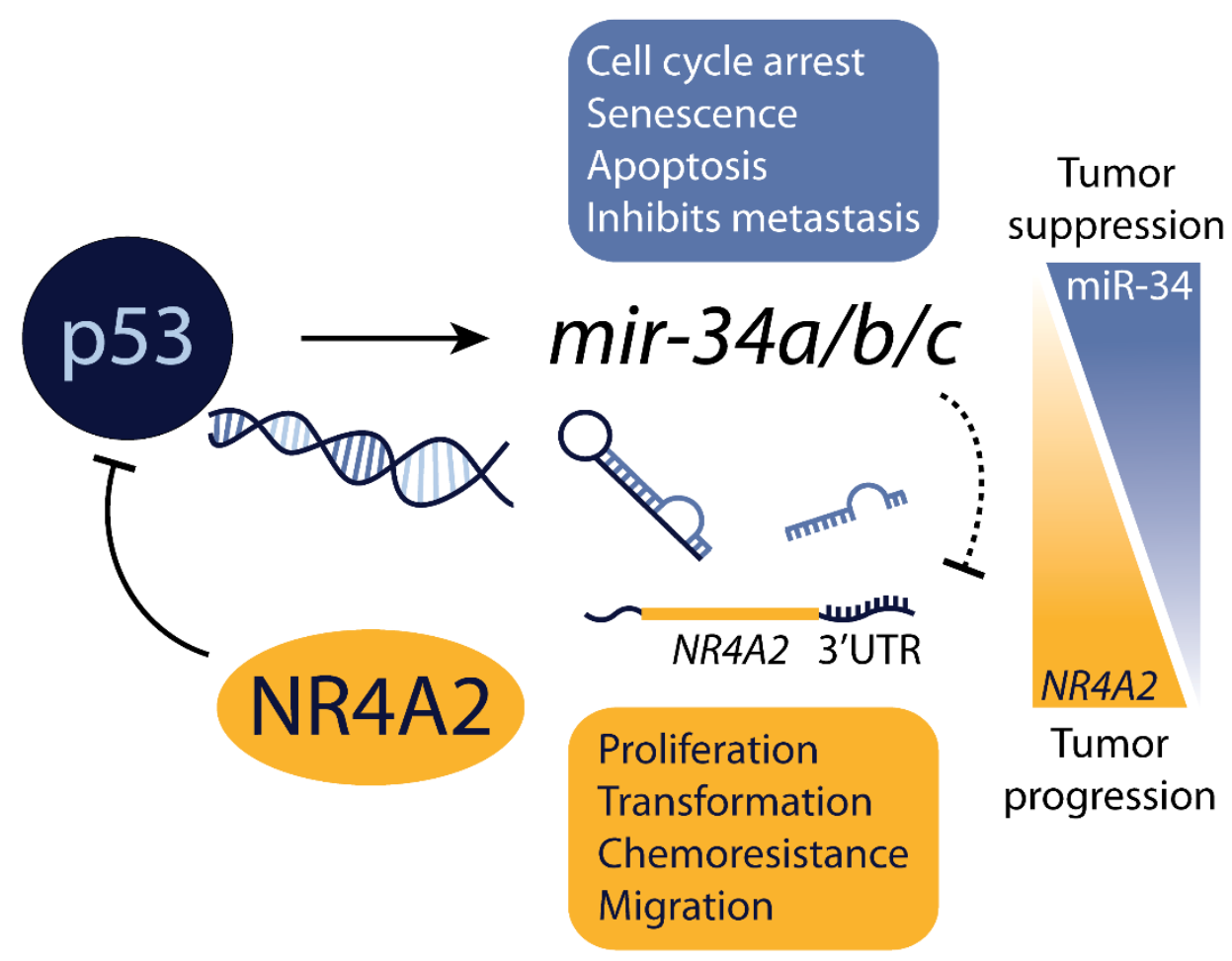

\section{Figure 3-7. Graphical summary.}

Here, we describe a novel miR-34 regulatory mechanism of $N R 4 A 2$ that can act downstream of p53 activation. Additionally, our studies extend the finding that NR4A2 can suppress p53 expression and transcriptional targets, including Nutlin-3a-induced activation of mir-34a. The p53-miR-34 regulation of NR4A2 may serve as a protective mechanism to prevent p53 suppression by NR4A2, in addition to suppressing other tumorigenic properties of NR4A2. The long arrow indicates activation; the blunt arrows indicate inhibition; the blunt arrow with the dotted line highlights the inhibition of $N R 4 A 2$ by miR-34 that indirectly activates $\mathrm{p} 53$. As tumors lose expression of mir-34, the expression and function of NR4A2 may become enhanced, shifting the balance from tumor suppression to tumor progression. 
by miR-34, a well-described tumor suppressor-like miRNA that targets genes involved in cancer progression and is increasingly being exploited for its therapeutic advantage [233]. Whereas other putative miRNAs were identified as targeting $N R 4 A 2$, we focused on the miR-34 family because of the known role of NR4A members in inhibiting p53 [95, 150] and the positive regulation of miR-34 by p53 [70], thereby framing our discovery of the regulation of NR4A2 by miR-34 as a possible feedback mechanism. The miR-34 isoforms, predominantly mir-34a, have tumor suppressor functions in multiple cancer types, which is sometimes attributed to their p53 status [253, 254], though the mechanisms of chromosomal deletion or epigenetic silencing are also major contributors $[73,74,237,238]$, and miR-34 expression is prognostic for patient outcome or relapse [254-257]. Likewise, several studies have demonstrated the ability of miR-34a restoration to sensitize cells to chemotherapeutic agents, including erlotinib [258], Adriamycin/doxorubicin [259], and 5-FU [260]. By using a colorectal cancer cell line pair that was sensitive or resistant to 5-FU, Akao et al. demonstrated that miR-34a was significantly downregulated in the resistant cells, and this was accompanied by increased expression of SIRT1, a target of miR-34. The expression of endogenous miR-34a remained low in 5-FU-resistant cells treated with 5-FU, and the restoration of miR-34a or knockdown of SIRT1 in the resistant cells overcame their resistant phenotype. In light of this observation, it would be interesting to study the relationship of miR-34 and NR4A2 with respect to chemoresistance, as increased NR4A2 expression is correlated with worse patient outcomes with regard to 5-FU therapy [93, 94, 230, 231].

By using an in vitro luminescence reporter-based screening assay and three computer-based prediction algorithms [242-245], we identified miR-34c as a putative regulator of $N R 4 A 2$ via its 3' UTR region (Figure 3-1 and Table 3-1). This regulatory effect was confirmed by using mutagenesis of the predicted miRNA recognition element that is complementary to the miR-34 seed region. Other miRNAs from this screen have been evaluated using computer-based and mutagenesis approaches (Table 3-1), and the functional significance of these miRNA regulators of $N R 4 A 2$ remains to be elucidated in future studies. Using publically available data, we found a weak negative (inverse) correlation of miR-34a with $N R 4 A 2$ (Figures A-1 and A-2), which was similar to the correlations of miR-34a with other known miR-34 target genes, such as $A X L$ [246] and SIRT1 [235], although NOTCH1 [259] did not show a negative correlation as expected. Interestingly, the patient sample data demonstrated two populations of TP53-expressing cells, and when we considered only those patient tumor samples with normal levels of TP53, the correlation became stronger. As we would hypothesize, there was a positive correlation of $N R 4 A 2$ with $A X L$ and SIRT1 since these genes share a common miRNA regulator, miR-34. Consistent with our data in Figures 3-4d and 3-5e, the level of $N R 4 A 2$ was negatively correlated to that of TP53 (Figure A-2).

The next objective was to determine whether miR-34 could regulate endogenous NR4A2, at both mRNA and protein levels. Indeed, overexpression of mature miR-34 isoforms reduced the endogenous levels of NR4A2 mRNA and protein in both wild-type and p53-deficient cells (Figures 3-2 and A-4). This would be expected, as forced expression of exogenous miR-34 bypasses the requirement for p53. Additionally, miR-34 overexpression enhanced the p53 activity in wild-type cells, probably because of the 
known regulatory effect of miR-34 on negative regulators of p53 (Figures 3-2 and A-3). This effect is largely the result of increased p53 protein levels rather than substantial changes in TP53 transcript levels (Figure A-3). The effect of miR-34 on NR4A2 was not as dramatic in the HCT116 p53 wild-type cells, especially at a later time point ( $24 \mathrm{~h})$ (Figure 3-2c), possibly because the lower levels of $N R 4 A 2$ in these cells no longer respond to exogenous miR-34 expression after the initial transfection, although reduced protein expression was observed at $48 \mathrm{~h}$ post-transfection (Figure 3-2e). This effect could also result from the delicate balance of $N R 4 A 2$ expression being regulated directly by miR-34 or indirectly through cellular stress. Correspondingly, RKO p53 wild-type cells had higher levels of endogenous NR4A2 and responded better to miR-34 mimics than their p53-deficient counterpart (Figure A-4), further supporting the notion that endogenous levels of $N R 4 A 2$ affect its response to exogenous miR-34.

To further understand the importance of miR-34 regulation of NR4A2 in the cellular context, we examined the relationship between endogenous miR-34 and endogenous $N R 4 A 2$ by using our isogenic cell line pairs that possess wild-type or deleted TP53. One interesting observation was that treating HCT116 cells with Nutlin-3a at an early time point $(24 \mathrm{~h})$ led to increased $N R 4 A 2$ expression, but the opposite effect was observed at a later time point (48 h) (Figure 3-3d). Consistent with this result, the NR4A2 protein expression was also reduced after $48 \mathrm{~h}$ of Nutlin-3a treatment in WT cells (Figure 3e). The NR4A family are immediate-early genes that are inducible by many stimuli, including cell stress and cytotoxic agents [78], whereas Nutlin-3a has a p53independent role and can induce a DNA damage response leading to cell cycle arrest [261]. One of these properties might account for the earlier induction of NR4A2 by Nutlin-3a, as the NR4A family mediates DNA double-strand break repair [201]. This hypothesis could be further tested by using MDM2-deficient cells to determine if these effects are independent of the primary role of Nutlin-3a in Mdm2 inhibition.

Finally, we investigated the previously reported inhibitory effect of NR4A2 on p53 activation [95] to determine if this regulation had an effect in the cellular context. We confirmed that overexpression of exogenous $N R 4 A 2$ can inhibit p53 induction of target genes in response to Nutlin-3a treatment (Figure 3-4) and that the opposite effect is seen when NR4A2 expression is reduced by using siRNA (Figure 3-5). Our data indicate that this effect might be achieved at least partially through NR4A2 suppression of TP53/p53 gene expression. Further investigation is warranted to determine if this suppression results from direct or indirect repression of the TP53 promoter. The suppressive effect of NR4A2 on the expression of p53 and its transcriptional targets was not reflected in changes in the binding of p53 to its target gene promoters. However, this finding may reflect the sensitivity of the assay, as even in the presence of NR4A2, enough p53 may be present to saturate the promoter response elements during Nutlin-3a treatment. It is also possible that NR4A2 is not inhibitory to p53 binding but is repressive of p53 transactivation at its target gene promoters.

NR4A2 inhibition of the p53 response has previously been investigated only with respect to the genotoxic agent doxorubicin; here, we have demonstrated a similar effect using a more targeted p53 activator. When cells overexpressing $N R 4 A 2$ were subjected to 
prolonged treatment with Nutlin-3a, we observed a substantial rescue of cells from Nutlin-3a-induced inhibition of proliferation (Figure 3-6), suggesting that the inhibitory effect of $N R 4 A 2$ on p53 activation is responsible for phenotypic observations that are indicative of $\mathrm{p} 53$-induced tumor suppression. Interestingly, in cells overexpressing $N R 4 A 2, N R 4 A 2$ expression was increased in Nutlin-3a-treated cells after prolonged treatment (Figure 3-6c). This effect may be attributable to the ability of $N R 4 A 2$ to repress the inhibition of cell proliferation, and as the selective pressure of Nutlin-3a is applied to all the cells, those cells expressing the highest levels of $N R 4 A 2$ persist in culture.

In summary, our study identified and confirmed a novel regulation of NR4A2 by miR-34. This regulatory effect is observed in the context of p53 activation, and NR4A2 itself is able to repress the p53 response. These events can be regarded as a positive feedback loop for p53 (Figure 3-7), much like other miR-34 targets that also suppress p53 [235, 236]. In tumors expressing wild-type p53, this can serve as a means by which p53 can release itself from repression by NR4A2, further enhancing the p53 response. Alternatively, cells that contain p53 mutations or that overexpress $N R 4 A 2$ through other means may lack the p53-mediated suppression of NR4A2, allowing NR4A2 to perform other oncogenic activities through its role as a transcription factor. As tumors lose expression of mir-34, through promoter methylation or the loss of p53, the expression and function of NR4A2 may become enhanced, shifting the balance from tumor suppression to tumor progression (Figure 3-7), though this may not be directly traceable because of the complexity of miRNA-mRNA regulatory networks.

\section{Materials and Methods}

\section{Cell culture}

All cell lines were grown in culture at $37^{\circ} \mathrm{C}$ in $5 \% \mathrm{CO}_{2}$. Human embryonic kidney 293 T cells (ATCC, Manassas, VA) were grown according to the manufacturer's protocol. Human colorectal carcinoma HCT116 wild-type and TP53-/- isogenic cell lines, which were derived from an adult male harboring a mutation in codon 13 of the ras protooncogene, were obtained from the Genetic Resources Core Facility at Johns Hopkins University School of Medicine (Baltimore, MD) and grown in McCoy's 5A medium (ATCC) supplemented with 10\% fetal bovine serum (Thermo Fisher Scientific Inc., Rockford, IL) and 1\% penicillin-streptomycin (Life Technologies, Carlsbad, CA). RKO human colon carcinoma (derived from a female harboring mutations in BRAF, NF1 and PIK3CA) and SW48 human colorectal adenocarcinoma (derived from a female harboring mutations in CTNNB1, FBXW7 and EGFR) (wild-type and TP53-/-) and HCT116 (CDKN1A-/- and BBC3-/-) isogenic cell line pairs [262-264] were obtained from Horizon Discovery (Cambridge, UK) and grown according to the manufacturer's protocol. Cells collected for RNA and protein extraction were detached with $0.05 \%$ trypsin-EDTA (Life Technologies). For all luminescence-based assays, the cells were plated using phenol-red-free DMEM or RPMI-1640 medium (Life Technologies). 


\section{3' UTR reporter plasmid and microRNA screen}

The NR4A2 3' UTR was cloned by GeneCopoeia, Inc. (Rockville, MD), directly downstream from a firefly luciferase (Fluc) gene under the control of an SV40 promoter in the pEZX-MT01 vector, which also contains a Renilla luciferase (Rluc) gene under the control of a CMV promoter (as a transfection control). This reporter construct (WT 3 UTR) was used to identify miRNAs that regulate Fluc activity through binding to the NR4A2 3' UTR and degradation or translational inhibition of fused Fluc mRNA. The $\mathrm{R} l u c$ activity was used to normalize the Fluc. The 293T cells were cotransfected for $48 \mathrm{~h}$ with the 3' UTR reporter plasmid and 75 cancer-related miRNAs selected from a previously described library [241] (Table A-1) by using Lipofectamine 2000 (Life Technologies) according to the manufacturer's protocol. Fugene 6 (Promega, Madison, WI) was used for transient cotransfection of reporter gene plasmids and miRNAs into HCT116 cells according to the manufacturer's instructions. Dual-Glo luciferase assays (Promega, Madison, WI) were performed to measure and calculate the ratios of firefly and Renilla luciferase activity. Luciferase activity was measured with an EnVision 2102 Multilabel Plate Reader (Perkin Elmer Inc., Waltham, MA).

\section{miRNA target prediction and mutagenesis}

Three miRNA target-prediction algorithms were used to identify putative miRNA regulators of NR4A2: TargetScanHuman, http://www.targetscan.org [242]; miRanda, http://www.microrna.org [243]; and PicTar, http://pictar.mdc-berlin.de/ [244]. By using these algorithms, a putative seed region was determined and mutated using site-directed mutagenesis (Mutagenex, Inc., Piscataway, NJ). Reporter constructs containing either the wild-type (WT 3UTR) or mutated (34mut) 3' UTR were used to demonstrate miR-34 specificity in the NR4A2 3' UTR.

\section{Molecular cloning}

NR4A2 cDNA was cloned into the pEXM12-3xFLAG (N-terminal) vector (GeneCopoeia, Inc.). We used the primers listed in Table A-2 to amplify 3xFLAGNR4A2 cDNA from pEX-3xFLAG-NR4A2 by PCR then subcloned it into a pSin-EF2IRES-Blast lentiviral expression vector (kindly provided by Dr. Mark E. Hatley). All DNA constructs were confirmed by Sanger sequencing. Lentiviral expression plasmids were packaged into viral particles using the psPAX2 packaging (Addgene plasmid \# 12260) and pMD2.G envelope (Addgene plasmid \# 12259) vectors.

\section{miRNA mimics, RNAi, and chemical treatments}

Exogenous expression of mature miRNAs was performed with mirVana mimics transfected using Lipofectamine RNAiMAX reagent (Life Technologies). All chemical 
treatments were performed in full growth medium containing $0.1 \%$ dimethyl sulfoxide (DMSO; as a negative control) (Thermo Fisher Scientific) or Nutlin-3a (Sigma Aldrich) as specified by the experimental design. Small interfering RNA (siRNA) was obtained from Dharmacon (Lafayette, CO) and transfected at a concentration of $20 \mathrm{nM}$ using Lipofectamine RNAiMAX reagent.

\section{RNA extraction and quantitative real-time PCR}

RNA was extracted using Maxwell simplyRNA kits and a Maxwell 16 Instrument (Promega). For the experiments that used miRNA mimics, the total RNA (including small RNAs) was extracted from the collected cells using miRNeasy Mini kits (Qiagen) in accordance with the manufacturer's instructions. RNA concentrations were measured using a NanoDrop 8000 UV-Vis Spectrophotometer (Thermo Fisher Scientific). All cDNA used in mRNA and miRNA quantitative real-time PCR (qPCR) analyses was synthesized from extracted RNA by using the SuperScript VILO cDNA synthesis kit (Life Technologies) according to the manufacturer's protocol. The mRNA and miRNA expression data were generated using Applied Biosystems TaqMan assays (20x) and Fast Advanced Master Mix (Life Technologies). Thermal cycling for qPCR was performed with an Applied Biosystems 7900HT Fast Real-Time PCR system (Life Technologies) in accordance with the TaqMan Fast protocol.

\section{Protein extraction and Western blot analysis}

Protein was isolated by incubating cells in Pierce RIPA lysis buffer with added Halt Protease Inhibitor cocktail (Thermo Fisher Scientific) for $30 \mathrm{~min}$ on ice then sonicating the lysate for $10 \mathrm{~s}$ at 50\% amplitude to shear the DNA. The protein concentration was measured using the Pierce BCA Protein Assay (Thermo Fisher Scientific) according to the manufacturer's instructions.

Protein lysates were resolved on NuPAGE 4-12\% SDS-PAGE gradient gels (Life Technologies). After electrophoresis was completed, the proteins were transferred from the gels to nitrocellulose membranes with an iBlot dry transfer system (Life Technologies). Protein gels used for detection of NR4A2 protein were transferred to polyvinylidene fluoride (PVDF) membranes using wet transfer for $1 \mathrm{~h}$ at $100 \mathrm{~V}$ constant voltage. The specific antibodies used were rabbit anti-NR4A2 (Santa Cruz Biotechnology, sc-5568; M-196) (Figure A-8), mouse anti-p53 (Santa Cruz Biotechnology, sc-65334; B-P3), rabbit anti-Sirt1 (Santa Cruz Biotechnology, sc-15404; H-300), goat anti-PUMA $\alpha$ (Santa Cruz Biotechnology, sc-19187; N-19); rabbit anti-acetyl-p53 (Lys382) (Cell Signaling, Danvers, MA, \#2525); mouse anti-Gapdh (Ambion, Life Technologies); mouse anti-p21 (Oncogene Research Products, Boston, MA, OP64); mouse anti- $\beta$-actin (Sigma Aldrich, A5441; clone AC-15); and mouse antiFLAG (Sigma Aldrich, F1804; clone M2). All antibodies were diluted in Odyssey Blocking Buffer (LI-COR Biotechnology, Lincoln, NE). The secondary antibodies were 
goat anti-rabbit and goat anti-mouse IRDye $800 \mathrm{WC}$ or IRDye 680LT (LI-COR). All Western blot imaging was conducted using a LI-COR Odyssey Infrared Imaging System.

\section{Chromatin immunoprecipitation (ChIP)}

HCT116 cells transduced with pSin-EF2-IRES-Blast empty vector (EV) or $3 x$ Flag-NR4A2 lentivirus were grown in flasks and treated with vehicle (DMSO, 0.1\%) or Nutlin-3a $(10 \mu \mathrm{M})$ for $6 \mathrm{~h}$. The chromatin was then crosslinked with $1 \%$ formaldehyde for $10 \mathrm{~min}$. Cell extracts were digested for $10 \mathrm{~min}$ with 50 units of micrococcal nuclease (New England Biolabs, Ipswich, MA) at $37^{\circ} \mathrm{C}$ and further sonicated to yield sheared DNA fragments with an average length of 200 to 1000 base pairs. The sonicated samples were centrifuged to pellet the cell debris, and the supernatant was diluted 7-fold with ChIP dilution buffer (0.01\% SDS, 1.1\% Triton X-100, 1.2 mM EDTA, 16.7 mM Tris$\mathrm{HCl}, \mathrm{pH} 8.1,167 \mathrm{mM} \mathrm{NaCl}$, and protease inhibitor cocktail). The samples were precleared with ChIP-grade Protein G agarose beads (Cell Signaling, \#9007) in ChIP dilution buffer (1:1 ratio). Diluted supernatant $(100 \mu \mathrm{L})$ was reserved as input $(10 \%)$ for each treatment. Chromatin $(1 \mathrm{~mL})$ was used for each immunoprecipitation and was incubated overnight at $4^{\circ} \mathrm{C}$ with mouse anti-p53 (Santa Cruz Biotechnology, sc-126; DO1) or control mouse IgG. The antibody-protein-DNA complexes were precipitated by incubation with Protein $\mathrm{G}-$ agarose beads for $2 \mathrm{~h}$ at $4^{\circ} \mathrm{C}$. The protein-DNA complexes were eluted from the beads with elution buffer (1\% SDS, $\left.0.1 \mathrm{M} \mathrm{NaHCO}_{3}\right)$. The crosslinks were reversed, and DNA was eluted from the protein-DNA complexes by adding 200 $\mathrm{mM} \mathrm{NaCl}$ and incubating at least overnight at $65^{\circ} \mathrm{C}$. DNA was recovered and purified after protein digestion with Proteinase $\mathrm{K}$ at $45^{\circ} \mathrm{C}$ for $2 \mathrm{~h}$ using the Qiagen DNeasy Blood \& Tissue Kit (Qiagen Cat. No: 69506). Quantitative real-time PCR was performed to determine the changes in p53 occupancy at various known sites of p53 binding. The double-negative controls were nonspecific antibody (normal mouse IgG) and primers coding for regions that do not interact with $\mathrm{p} 53$. The thermal cycling conditions were $95^{\circ} \mathrm{C}$ for $10 \mathrm{~min}$ followed by 45 cycles of $25 \mathrm{~s}$ at $95^{\circ} \mathrm{C}, 30 \mathrm{~s}$ at $60^{\circ} \mathrm{C}$, and $30 \mathrm{~s}$ at $72^{\circ} \mathrm{C}$. The primers used are listed in Table A-2.

\section{Cell viability assays}

Real-time cell growth in response to the various treatments was measured as the degree of cell confluence in culture plates and was determined using an IncuCyte ZOOM live-cell imaging system (Essen BioScience, Ann Arbor, MI). Cell proliferation curves were plotted using confluence values at specified time points for each treatment.

\section{Analyses of expression data from online databases}

The RNASeq and miRNASeq dataset of 164 patients with rectum adenocarcinoma was acquired from the TGCA Research Network: http://cancergenome.nih.gov/. After $\log 2$ transformation of reported normalized_count 
for mRNA expression and read_per_million_miRNA_mapped for mature miRNA expression, a subset of 97 samples with data generated from the HiSeq platform (for a consistent and reliable comparison) was investigated. The correlations between the expression of miR-34a and $N R 4 A 2$, as well as other published miR-34 targets- $A X L$ [246], NOTCH1 [259], and SIRT1 [235] — and TP53 were determined by the regression analysis using Stata software (College Station, TX).

\section{Statistical analyses}

All experiments were performed at least three times, and the independent replicates are represented as mean \pm standard deviation. Data normalization, statistical tests used, and representations of $P$-value are indicated for each figure in the corresponding legend. 


\section{CHAPTER 4. INVESTIGATING THE EXPRESSION AND FUNCTION OF NR4A NUCLEAR RECEPTORS IN RHABDOMYOSARCOMA}

\section{Introduction}

Rhabdomyosarcoma (RMS) is the most common soft tissue sarcoma in children, affecting 350 children and adolescents per year in the United States [265]. RMS is thought to be derived from the skeletal muscle lineage and of the four subtypes characterized, two primarily affect children and adolescents-embryonal RMS (ERMS) and alveolar RMS (ARMS). ERMS is more common and is less aggressive, with these patients usually being younger and having a much better prognosis [266]. Highly aggressive ARMS is typically characterized by a fusion gene of $P A X 3 / 7-F O X O 1$ in $80 \%$ of cases [267], forming a transcription factor capable of driving oncogenic behavior in ARMS [268]. Additionally, other molecular signaling defects have been attributed to RMS pathogenesis, including Ras, p53, and Sonic hedgehog (Shh) pathways [269].

The NR4A orphan nuclear receptor (NR) family, particularly NR4A1 (Nur77) and NR4A2 (Nurr1), has been predominantly studied in adult cancers where higher expression of both have been observed [86, 270]. Gene knockdown of NR4A1 generally inhibits cell growth and increases apoptosis [86], explaining its importance in cell proliferation and survival. NR4A1 expression promotes proliferation in a DNA-binding and transactivation-dependent manner [271]. Conversely, in hematological malignancies, $N R 4 A 1$, along with $N R 4 A 3$ (Nor-1), is found to be downregulated or silenced in mouse and clinical samples [46]. In agreement with this, NR4A1 and NR4A3 were found to regulate the expression of many of the same genes [98], indicating their functional redundancy.

Less is known about how NR4A2 mediates cancer hallmarks. NR4A2 has been shown to play a role in anchorage-independent cell growth [88, 89]. Also, there is evidence that NR4A2 can mediate cell migration [90], and others show that NR4A2 can mediate cell proliferation and xenograft tumor growth [91, 92]. High expression of NR4A2 is found in squamous cell carcinoma (SCC) compared to normal tissues from patients, leading to increased resistance to 5-fluorouracil [93]. This suggests that NR4A2 has pro-survival roles or effects on drug resistance $[93,94]$. This could be explained partly by an interaction with p53, in which NR4A2 inhibits p53 oligomerization to suppress its transactivation of target gene $B A X$, thus protecting cells from doxorubicininduced apoptosis [95].

Much of these effects are attributable to the interaction of NR4As with various oncogene and tumor suppressor pathways, as well as downstream regulation of NR4A target genes [45]. The implications of NR4A expression and function in pediatric cancers are not known, although we hypothesize that the NR4A receptors will have similar functions in rhabdomyosarcoma. In this study, we assessed the expression of NR4A NRs in normal muscle and rhabdomyosarcoma cell lines. We found that, compared to normal muscle cells, the NR4A NRs are overexpressed in cell lines of RMS origin. When using 
siRNA to knockdown the expression of the NR4A members, we found that NR4A1 and $N R 4 A 2$ were able to affect cancer-like properties. Both $N R 4 A 1$ and NR4A2 knockdown caused a decrease in cell proliferation, with $N R 4 A 2$ having a greater effect. This observation was also supported by an increase in the percentage of cells in the G1 phase of the cell cycle. Only NR4A2 had an effect on cell migration, while both $N R 4 A 1$ and $N R 4 A 2$ knockdown decreased colony formation. Lastly, we performed a DNA microarray to assess the overlapping gene expression profiles among the three NR4A receptors in RMS. We found that knockdown of $N R 4 A 1$ and $N R 4 A 3$ had similar transcriptome profiles, while $N R 4 A 2$ knockdown had a unique expression signature. Using this transcriptome data to further understand the unique and shared target genes of the NR4A receptors, particularly those regulated by NR4A2 to affect cell migration, will offer new insights into their roles in cancer and metastasis and the potential for therapeutic manipulation by small molecules or miRNAs.

\section{Results and Discussion}

\section{NR4A nuclear receptors are overexpressed in RMS}

As summarized by Mohan et al. [270], the NR4A receptors have been shown to be highly expressed in many tumor types, mostly those of adult origin, including bladder, breast, colon, liver, and lung cancers. We first sought to determine the expression of the NR4A subfamily members in RMS cell lines. When comparing the levels of mRNA expression, we found that all three NR4As had higher expression in the RMS cell lines (RD, Rh41, and Rh30) in comparison to normal skeletal muscle cells (Figure 4-1). The expression of both $N R 4 A 1$ and $N R 4 A 2$ are higher in the cell models representative of ARMS, while $N R 4 A 3$ was expressed equally among the three cell lines. Considering the known roles of NR4A1 and NR4A2 in promoting cancer phenotypes, such as proliferation and migration, it is interesting to note that these two NR4A members were higher in cell lines of the more aggressive RMS subtype. With the multiple pathways known to regulate NR4A expression, it is possible that increased activity of these signaling cascades could be the cause of the NR4A overexpression and this remains to be determined.

\section{Knockdown of $N R 4 A 1$ and $N R 4 A 2$ decreases cell proliferation}

We next sought to determine the roles that the overexpressed NR4A receptors have in the RMS cell proliferation. Using Rh30, an RMS cell line of the ARMS subtype which had the highest expression of the NR4A receptors, we transfected siRNA to knockdown the expression of each NR4A receptor (Figure 4-2a). After 48 h of siRNAmediated knockdown, we began monitoring cell proliferation using real-time confluence measurement. We found that knockdown of $N R 4 A 1$ or $N R 4 A 2$ was able to reduce the cell proliferation, with $N R 4 A 2$ knockdown having a greater effect (Figure 4-2b). This data 


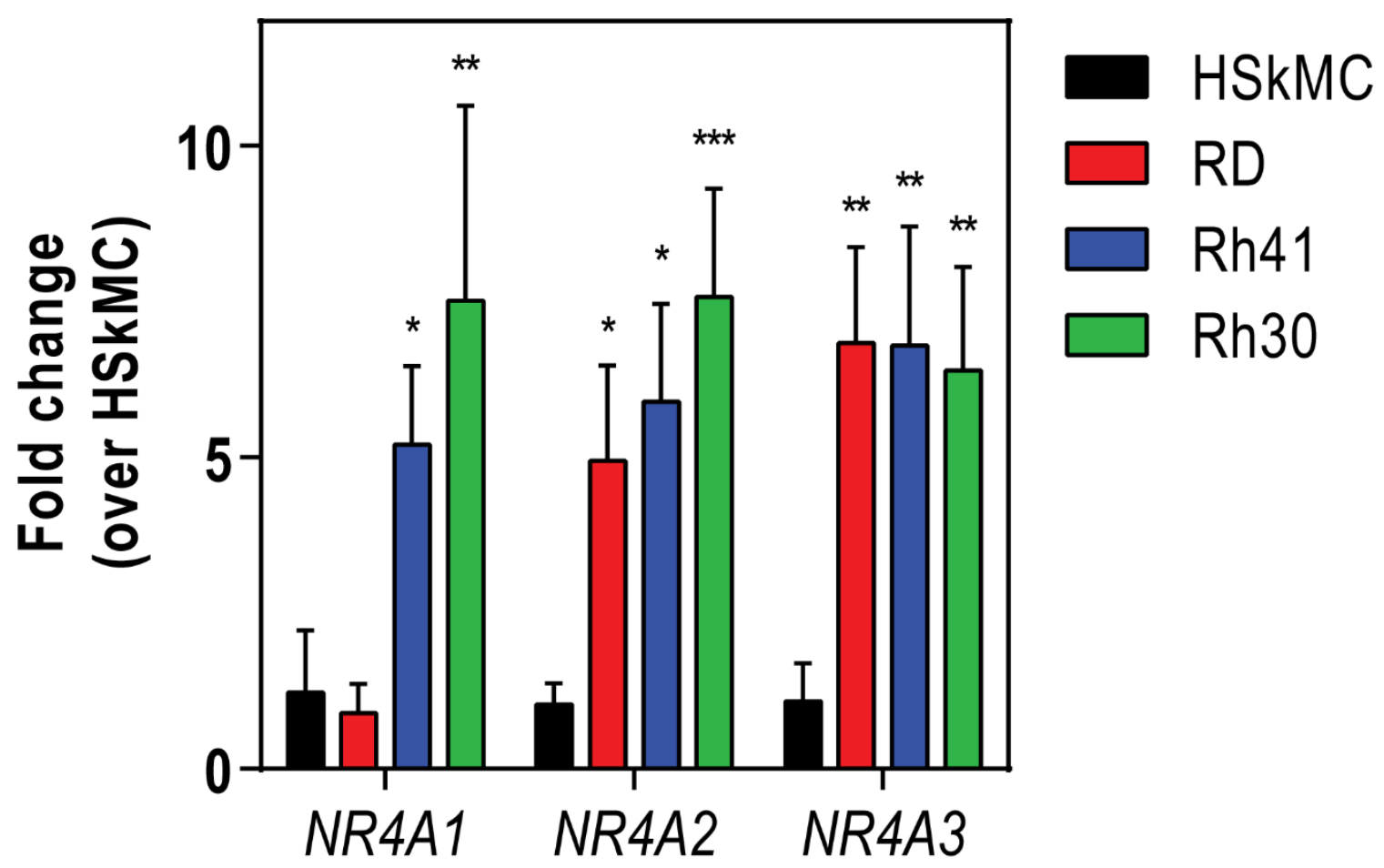

Figure 4-1. NR4A nuclear receptors are overexpressed in rhabdomyosarcoma cell lines.

The expression of $N R 4 A 1, N R 4 A 2$, and $N R 4 A 3$ was determined using Taqman qPCR probes. The expression of each gene was normalized to GAPDH as an internal control. The data is presented as the fold-change over HSkMC cells (as determined by $2^{-\Delta \Delta C t}$ ). The statistical significance of the results was calculated using a two-way ANOVA and Dunnett's test for multiple comparisons. ***, $P \leq 0.001 ; * *, P \leq 0.01 ; *, P \leq 0.05$. 
a.

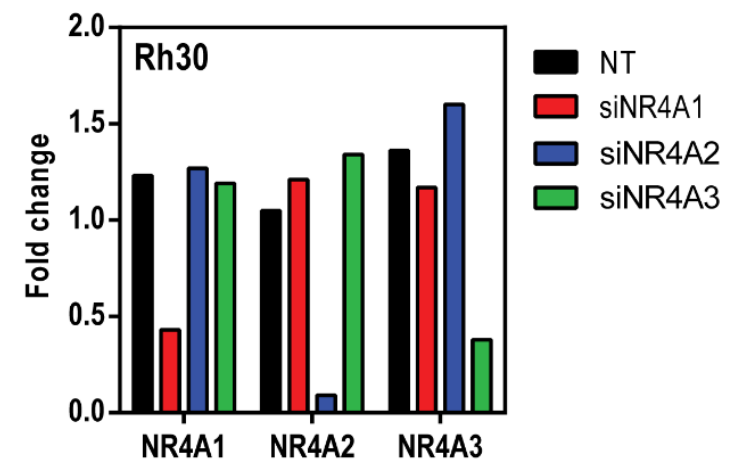

C.

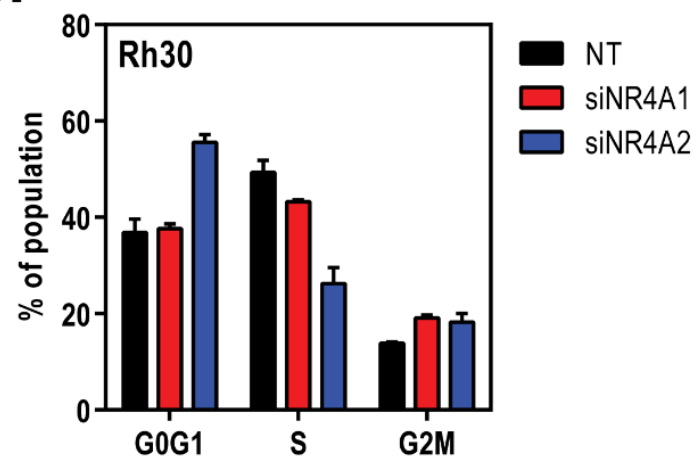

b.

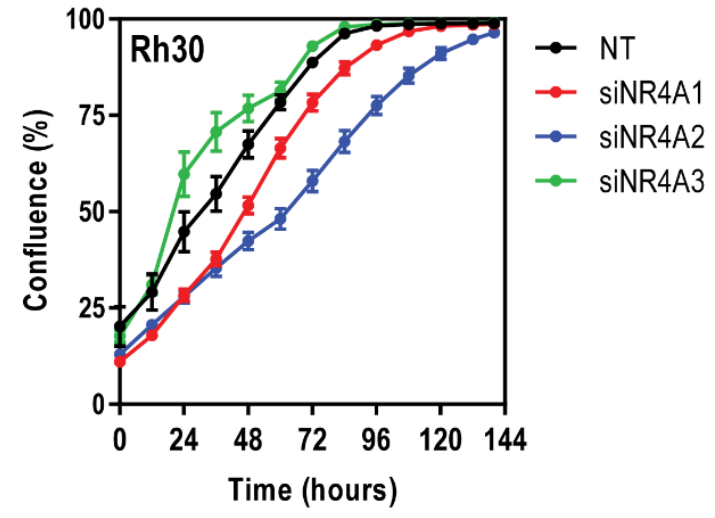

d.

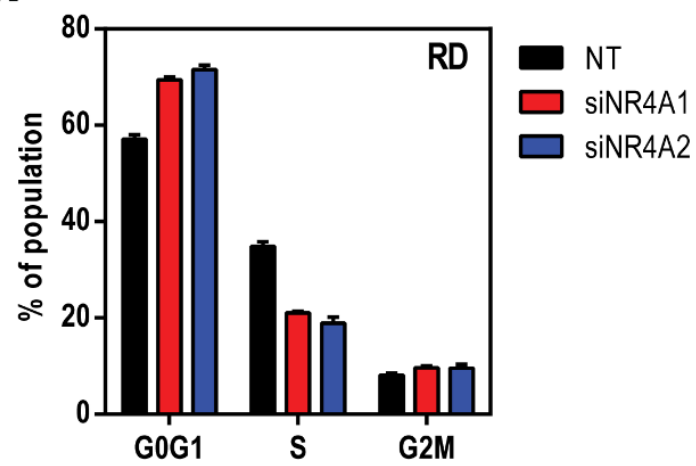

Figure 4-2. Knockdown of $N R 4 A 1$ and $N R 4 A 2$ decreases cell proliferation.

Rh30 cells were transfected for $48 \mathrm{~h}$ with $50 \mathrm{nM}$ non-targeting (NT) or siRNA against the NR4A genes. (a) The knockdown of expression was confirmed using Taqman qPCR probes. (b) Cells were then plated for real-time confluence determination using an IncuCyte HD imaging system. Rh30 (c) or RD (d) cells were transfected for $48 \mathrm{~h}$ with NT, siNR4A1, or siNR4A2 $(25 \mathrm{nM})$ and DNA content was determined using propidium iodide (PI) staining and flow cytometry analysis. 
corresponds with the known oncogenic-like effects that NR4A1 and NR4A2 demonstrate in adult cancer types [86, 270].

Additionally, we determined the cell cycle profile of Rh30 and another RMS cell line, $\mathrm{RD}$, following knockdown of $N R 4 A 1$ or $N R 4 A 2$. We determined that following siRNA transfection, the percentage of cells in the G1 phase of the cell cycle was increased with a corresponding decrease in the S-phase cell population (Figure 4-2c, d), which is indicative of a decreased proliferative capacity. For the instance of $N R 4 A 1$, this is likely explained by the regulation of target genes such as CCND2 (cyclin D2) [16], which plays a critical role in the transition of cells from the G1 to $S$ phase of the cell cycle. This is also a possible explanation for $N R 4 A 2$, as it has been previously suggested to regulate $C C N D 1$ (cyclin D1)-dependent proliferation of lung cancer cells [91].

\section{Knockdown of $N R 4 A 2$ decreases cell migration}

Next, we sought to characterize the effects of NR4A knockdown on cell migration using several assays. We first used siRNA to knockdown each NR4A member in RD cells (Figure 4-3a). Following $48 \mathrm{~h}$ of siRNA-mediated knockdown, we plated the cells for a wound healing assay. Using real-time imaging, we monitored the wound for $96 \mathrm{~h}$ total, and found that only knockdown of $N R 4 A 2$ was able to impair the ability for RD cells to efficiently reconstitute the wound (Figure 4-3b, c). Additionally, Rh30 cells were transfected with siRNA for $48 \mathrm{~h}$ and cell migration was determined using a Boyden chamber assay in which we allowed the cells to migrate from a serum-free environment towards a $10 \%$ serum-containing environment. Following $24 \mathrm{~h}$ of migration, we determined that siNR4A2 transfection was also able to attenuate the amount of cell migration through the membrane, which was quantified using image analysis software (Figure 4-3d, e). These two assays indicate that $N R 4 A 2$ is able to mediate the migratory capacity of RMS cells, which is in agreement with previous published data in bladder cancer [90]. In terms of cancer progression, this could indicate that $N R 4 A 2$ is involved in tumor metastasis in RMS. In contrast, other reports indicate an opposite and repressive role of NR4A2 on migration and invasion of adenocarcinoma cells [272], as well as lower expression in breast tissue from patients presenting with lymph node metastases [92]. These differences could be because of specific roles that are tissue-dependent, similar of that to NR4A1 having opposite roles in solid versus hematologic malignancies.

\section{Knockdown of $N R 4 A 1$ and $N R 4 A 2$ decreases colony formation}

Using the RD and Rh30 cell lines, we again transfected with siRNA targeting the NR4A members. After $48 \mathrm{~h}$, we performed a clonogenic assay by sparsely plating a minimal amount of cells and allowing them to form adherent colonies over the course of 10 days. We found that knockdown of $N R 4 A 1$ could slightly block the formation of cell clones in both cell lines, while knockdown of $N R 4 A 2$ could lead to a much greater attenuation of clonogenicity (Figure 4-4). This data also agrees with Figure 4-2, in that $N R 4 A 2$ knockdown had a greater effect on cell proliferation. This further supports the 
Figure 4-3. Knockdown of $N R 4 A 2$ decreases cell migration.

RD cells were transfected for $48 \mathrm{~h}$ with $50 \mathrm{nM}$ non-targeting (NT), siNR4A1, or siNR4A2.

(a) The knockdown of NR4A expression was confirmed using Taqman qPCR probes. (b)

$\mathrm{RD}$ cells were then plated into 24-well ImageLock plates and allowed to reach

confluence for $24 \mathrm{~h}$. A scratch-wound was then made uniformly in each well and

monitored in real-time using an IncuCyte HD imaging system to determine relative

wound confluence. (c) Representative images from real-time monitoring are shown. (d)

Rh30 cells were transfected with siRNA and we performed a transwell chamber assay

followed by crystal violet staining. (e) Migrated cells from 3 fields of each replicate were counted using ImageJ software. 

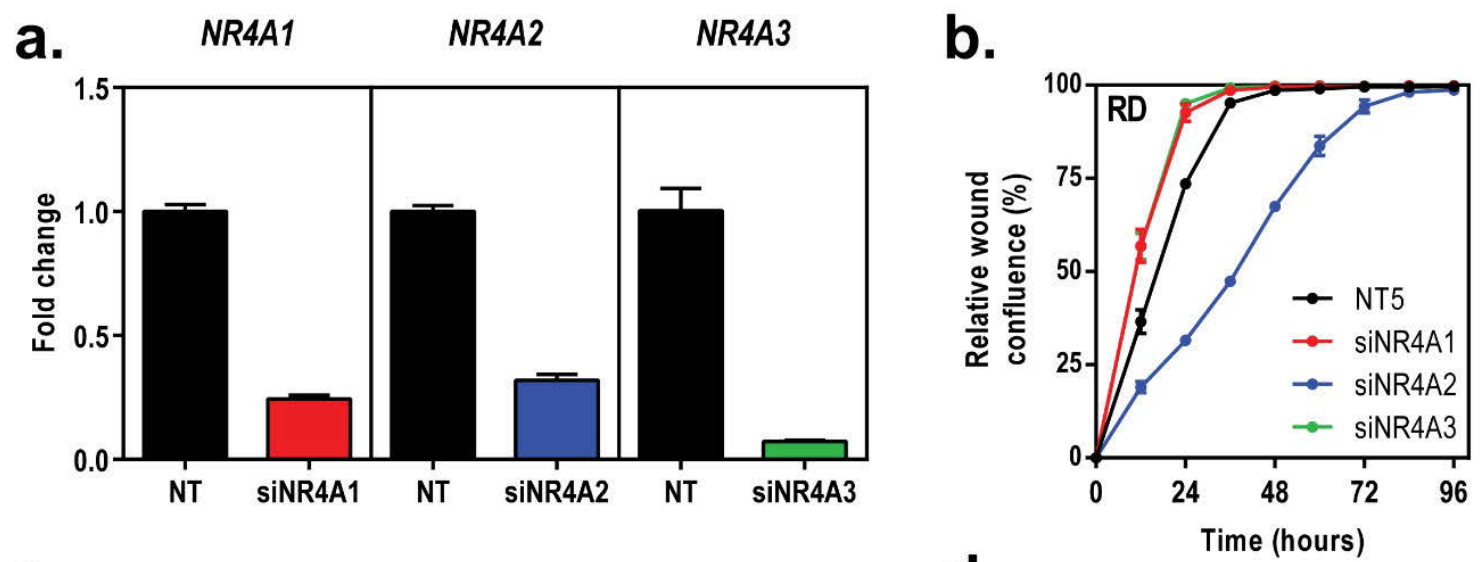

c.

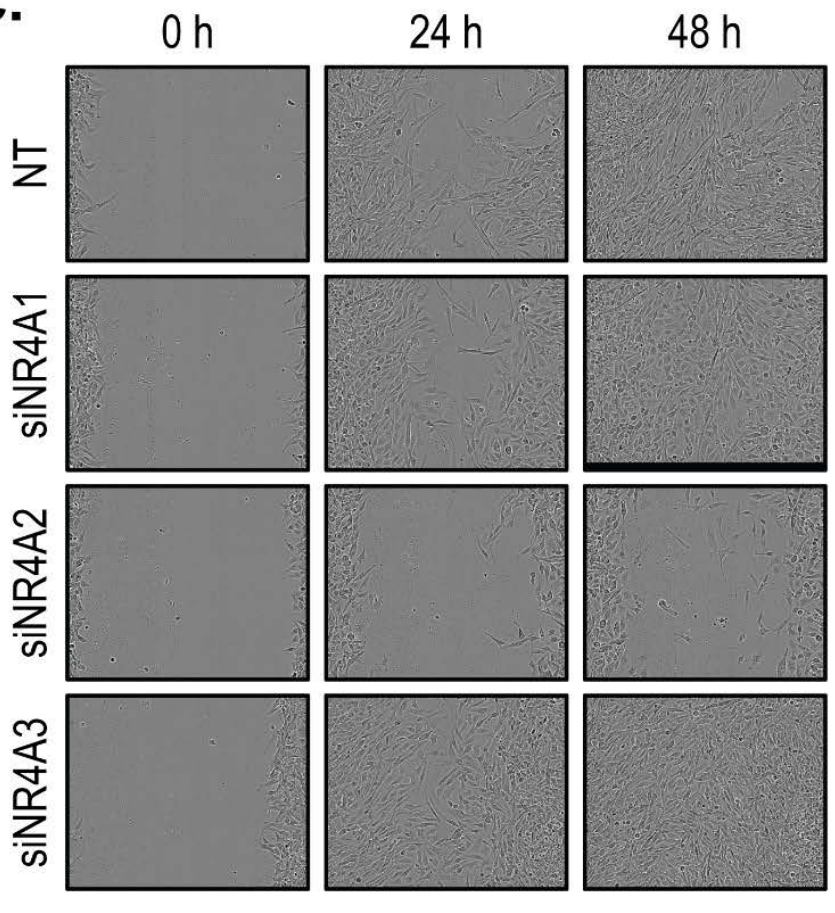

e.

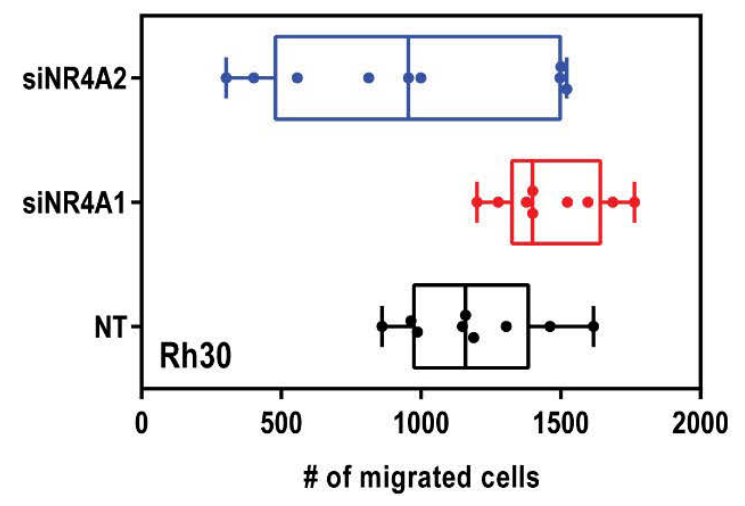

d.
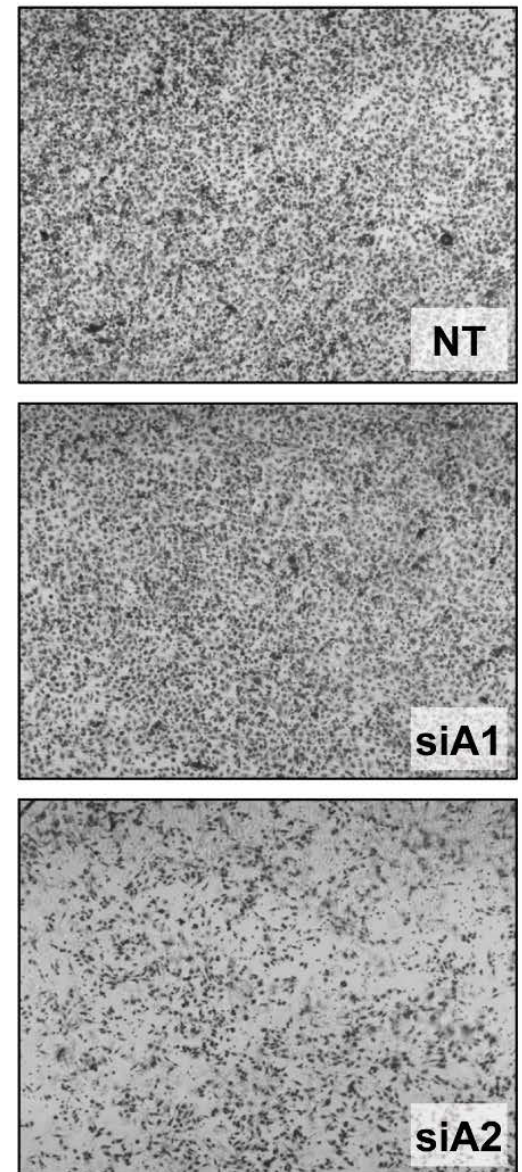

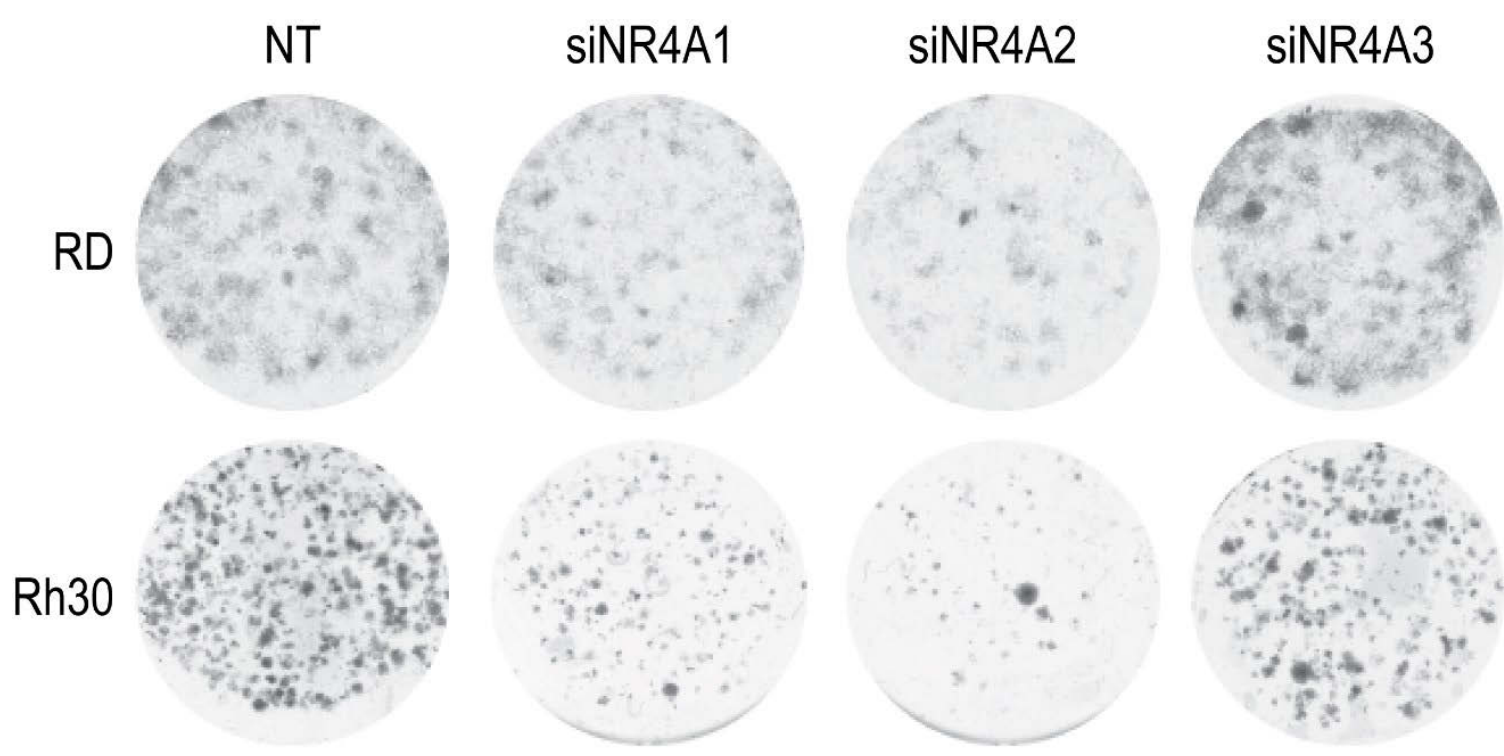

Figure 4-4. Knockdown of $N R 4 A 1$ and $N R 4 A 2$ decreases colony formation.

$\mathrm{RD}$ or Rh30 cells were transfected for $48 \mathrm{~h}$ with indicated siRNA $(50 \mathrm{nM})$. Cells $\left(2 \times 10^{3}\right)$ were then plated into 6-well plates in normal growth medium and allowed to grow for a period of 10 days, with medium changes every 2 days. Colonies were then fixed with methanol and stained with crystal violet to image colony formation. 
proliferative and pro-survival roles of the NR4A receptors, in particular NR4A2, and also is supported by the known roles of NR4A2 in transformation [88, 89]. The ability of $N R 4 A 2$ to affect anchorage-independent colony formation in RMS remains to be determined.

\section{$N R 4 A 2$ knockdown leads to an altered transcriptional profile}

It is well described that the pro-oncogenic functions of NR4A1 are carried out through its target genes, which include CCND2 [16], E2F1 [85], survivin [47], and TXNDC5 [48], but the contributions of NR4A2 to gene expression regulation in cancer are not well defined To date, a systematic transcriptome study in cancer has not been completed for the NR4A family, which could yield insights into shared and unique target genes of the NR4A family. To understand how the NR4A members, particularly NR4A1 and NR4A2, are affecting the cell proliferation and migration in RMS, we performed a microarray study in which we used siRNA to knockdown the expression of each NR4A member in either single, double, or triple knockdown combinations. Following $48 \mathrm{~h}$ of siRNA transfection, we confirmed the specificity and efficiency of the siRNA combinations (Figure 4-5a). We next performed a microarray study to determine the gene expression changes occurring under each condition. Principal component analysis (PCA) of the significantly altered genes indicated that the samples containing knockdown of NR4A2 clustered together, indicating their similarity of gene expression, while those containing knockdown of $N R 4 A 1$ or $N R 4 A 3$ also clustered together (Figure 4-5b). This supports the current understanding of the NR4A receptors in that NR4A1 and NR4A3 were previously shown to have redundant roles and regulate up to $97 \%$ of the same gene transcripts [98]. This finding is also interesting in that the $N R 4 A 2$ knockdown causes a shift in the gene expression profile of significantly altered genes, some of which may explain the unique effect that NR4A2 has on cell migration in this study (Figure 4-3). Also, when clustering the samples by significantly upregulated and downregulated genes, those samples with $N R 4 A 2$ knockdown clustered together (Figure 4-5c). Several of the genes significantly altered by $N R 4 A 2$ knockdown were involved in cell cycle and motility, and the direct regulation of these genes remains to be determined. Many of the genes significantly altered by $N R 4 A 2$ knockdown were increased in expression, which could indicate a mechanism of gene suppression by NR4A2.

\section{Conclusions}

In this study, we have further described the pro-oncogenic properties of the NR4A family, particularly $N R 4 A 1$ and $N R 4 A 2$. This body of data expands the known roles of this subfamily of NRs into the field of pediatric oncology, where little is known regarding the function of NR4A members. Using multiple phenotypic assays, we identified that $N R 4 A 1$ and NR4A2 can promote cancer cell properties such as proliferation through cell cycle regulation, migration, and survival and clonogenecity. Additionally, a systematic microarray study to identify NR4A regulated genes indicated that the effect of $N R 4 A 2$ depletion has drastic effects on gene expression, which might explain its unique role as a 
Figure 4-5. Gene expression following NR4A knockdown.

Rh30 cells were transfected for $48 \mathrm{~h}$ with siRNA for each NR4A receptor in single, double, or triple combinations (25 nM NR4A-specific siRNA, $75 \mathrm{nM}$ total siRNA per sample). (a) Knockdown efficiency and specificity was determined. The expression of $N R 4 A 1, N R 4 A 2$, and NR4A3 was determined using Taqman qPCR probes. The expression of each gene was normalized to $G A P D H$ as an internal control. The data is presented as the fold-change over non-targeting siRNA (as determined by $2^{-\Delta \Delta C t}$ ). (b) Principal component analysis was performed on the genes that were significantly upregulated or downregulated in any of the siRNA conditions. (c) Fold-change of significantly altered genes over non-targeting is presented as a heatmap ( $\log 2$ fold change; red $=+2.5$, blue $=$ $-2.5)$. 
a.

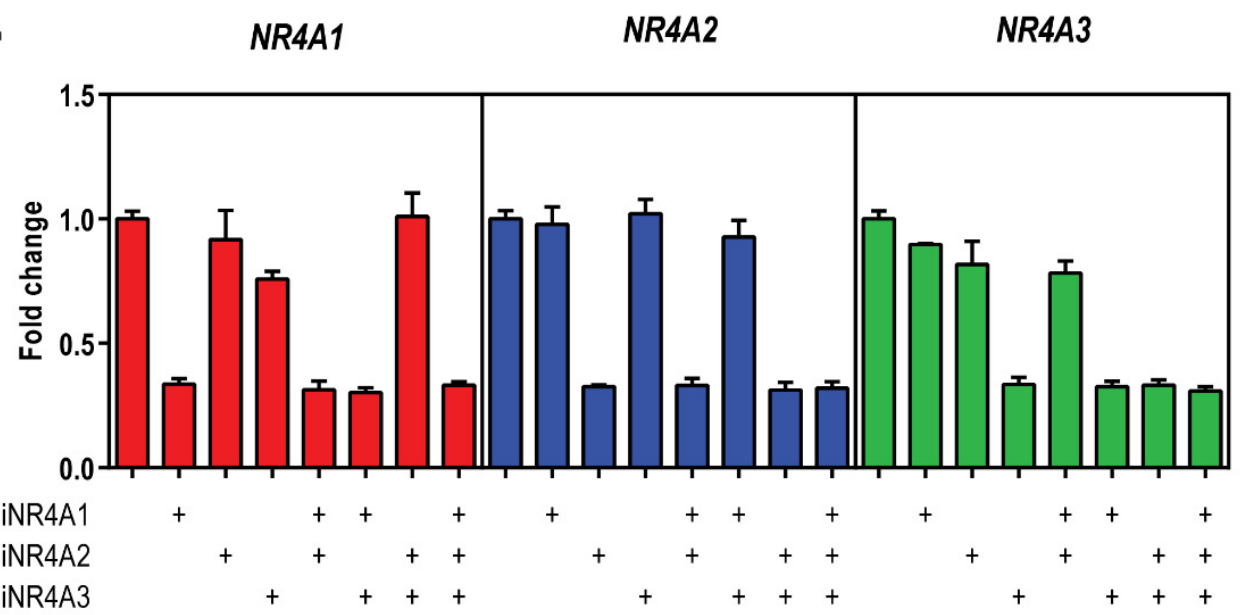

b.

PCA Mapping (Significant Genes)

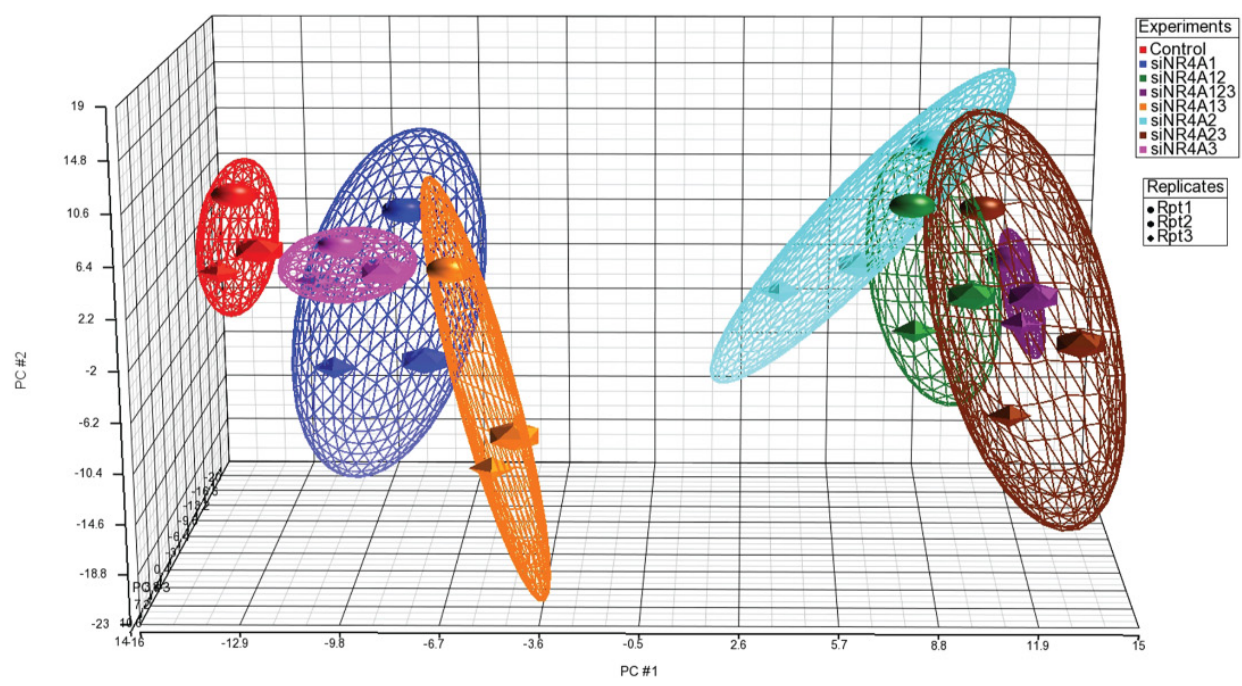

c.

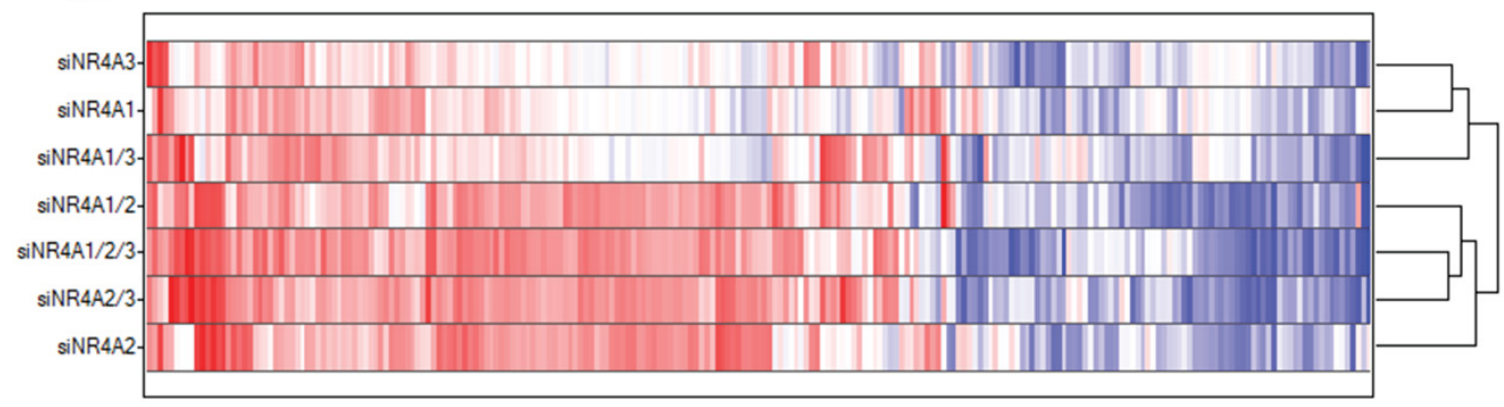


mediator of cell migration in the RMS cells. It remains to be determined which novel genes are critical for the effects observed following NR4A knockdown, and if these effects are through direct regulation of novel target genes at promoter binding sites.

\section{Materials and Methods}

\section{Cell culture}

All cell lines were grown in culture at $37^{\circ} \mathrm{C}$ in $5 \% \mathrm{CO}_{2}$. Rh30 and $\mathrm{RD}$ rhabdomyosarcoma cell lines (ATCC, Manassas, VA) were grown according to the manufacturer's protocol. Rh41 cells were provided by Dr. Peter Houghton and grown in RPMI-1640 medium (Life Technologies, Carlsbad, CA) supplemented with 10\% fetal bovine serum (Thermo Fisher Scientific, Rockford, IL). Human skeletal muscle cells (HSkMC; Cell Applications, Inc., San Diego, CA) were cultured according to manufacturer's guidelines. Trypsin (0.05\%)-EDTA (Life Technologies) was used to detach cells during passaging and cell collection for RNA and protein extraction. Cells were grown in the presence of $1 \%$ penicillin-streptomycin (Life Technologies) except during siRNA transfections.

\section{Transfection of siRNA, RNA extraction, and quantitative real-time PCR}

Small interfering RNA (siRNA) was obtained from Dharmacon (Lafayette, CO) and transfected at indicated concentrations using Lipofectamine RNAiMAX reagent (Life Technologies). Transfections were performed in the absence of antibiotics, and culture medium was changed after 16 hours of incubation with lipid-siRNA complexes. Maxwell simplyRNA kits and the Maxwell 16 Instrument (Promega, Madison, WI) were used to extract RNA from cell samples. RNA concentration was measured using a NanoDrop 8000 UV-Vis Spectrophotometer (Thermo Fisher Scientific). cDNA was synthesized from $2 \mu \mathrm{g}$ total RNA using the SuperScript VILO cDNA synthesis kit (Life Technologies) according to the manufacturer's protocol.. The mRNA expression was determined using Taqman gene expression assays and Fast Advanced Master Mix (Life Technologies). The Applied Biosystems 7900HT Fast Real-Time PCR system (Life Technologies) was used for qPCR thermal cycling.

\section{Cell proliferation and cell cycle assays}

Rh30 cells were transfected with siRNA $(50 \mathrm{nM})$ for $48 \mathrm{~h}$ and plated $\left(1 \times 10^{5}\right)$ into 6 -well plates to determine real-time cell proliferation in response to NR4A knockdown. Cell proliferation was measured as cell confluence in culture plates using an IncuCyte HD live-cell imaging system (Essen BioScience, Ann Arbor, MI). Cell proliferation curves were plotted using confluence measurements at specified time points for each siRNA transfection. Cells transfected with siRNA were also collected after $48 \mathrm{~h}$ after 
transfection and DNA content was assessed using propidium iodide (PI) staining. Briefly, $1 \times 10^{6}$ cells were washed with PBS and resuspended in PI solution $(0.05 \mathrm{mg} / \mathrm{mL}$ PI, $0.1 \%$ sodium citrate, $0.1 \%$ Triton X-100). Samples were then treated with RNAse for $30 \mathrm{~min}$ at room temperature, filtered through $40 \mu \mathrm{m}$ mesh, and analyzed by flow cytometry.

\section{Cell migration assays}

Wound healing assays were performed by plating cells into 24-well ImageLock plates (Essen BioScience) and allowing them to reach full confluence. A scratch-wound was made using the Essen BioScience Woundmaker to make a consistent scatch across the middle of the imaging field. Plates were then monitored in real-time for indicated time points and the ability of cells to migrate towards the center of the wound was determined using the Essen Cell Migration software module. Boyden chamber migration assays were performed by plating $2 \times 10^{4}$ cells with serum-free medium into transwell chambers with a pore size of $8 \mu \mathrm{m}$ (BD Biosciences, San Jose, CA). Chambers were placed in a 12 -well plate with $10 \%$ serum-containing medium, and cells were allowed to migrate towards the serum-containing medium for $24 \mathrm{~h}$. Transwell chambers were then fixed with methanol (Sigma Aldrich) for $10 \mathrm{~min}$ and stained with $0.4 \%$ crystal violet for $2 \mathrm{~h}$ (Sigma Aldrich) and mounted to a glass slide for light microscopy imaging. Three fields for each replicate were counted using ImageJ software.

\section{Colony formation assay}

A clonogenic assay was used to measure the ability of single cells to grow into cell colonies. After indicated siRNA transfection, $2 \times 10^{3}$ cells were plated into 6 -well plates in normal growth medium. Culture medium was replaced every 2 days, and colonies were allowed to form for a total of 10 days. Colonies were then fixed with methanol for $10 \mathrm{~min}$ and stained with $0.05 \%$ crystal violet for $2 \mathrm{~h}$ and plates were scanned.

\section{DNA microarray}

Rh30 cells were transfected with $25 \mathrm{nM}$ of NR4A-specific siRNA in single, double, or triple combinations (75 nM total siRNA) for $48 \mathrm{~h}$, then collected for RNA extraction. Total RNAs were amplified and labeled by using a One-Color Low Input Quick Amp Labeling kit (Agilent 5190-2305, Santa Clara, CA), followed by hybridizing to the SurePrint G3 Human GE $8 \times 60 \mathrm{~K}$ microarray (Agilent-028004) that contains 42,545 unique probes targeting 27,958 Entrez genes. Microarrays were scanned by using an Agilent array scanner (G2565CA) at $3 \mu \mathrm{m}$ resolution. Microarray data were extracted by Agilent Feature Extraction software (v10.5.1.1) using GE1_107_Sep09 protocol. The quantile normalization on log transformed background-subtracted signal intensity was performed among all samples, followed by comparison between sets of replicates from different experimental groups. The Student's t-test was used to determine the statistical 
significance of the difference between the paired samples from three replicates of each experiment. The expression of the gene was considered significantly different if $p<0.05$ and expression change is greater than two folds for at least one of the group comparisons. The data process and PCA analysis were performed using Partek software (St. Louis, MO). Microarray expression data files were deposited in the Gene Expression Omnibus (GEO) as series record GSE68458. 


\section{CHAPTER 5. DISCUSSION}

\section{NR4A Regulation by miRNAs in Cancer}

Many studies have suggested a pro-oncogenic role for the NR4A NRs and, as such, high expression of these NRs has been identified in multiple tumor types. The NR4A members are early induced genes, with increased expression during situations such as mitogenic stimulation, stress, or cell death [78]. Because of this, the cause for the overexpression of the NR4A members in cancer has been attributed to oncogenic signaling pathways [45], mostly those regulated by growth factors. The NR4A members are intertwined with these pathways and frequently can exert effects onto the signaling pathways as well, including inhibition of tumor suppressor signaling to further enhance cell proliferation and survival.

One area in which research is lacking for the NR4A receptors is in miRNA regulation of these NRs, particularly as it relates to cancer. Our lab recently reported that $N R 4 A 1$ is regulated by miR-124 [273], the first characterized miRNA regulator of NR4A1. Upon observation of decreased miR-124 and increased NR4A1 in medulloblastoma cells, we found that miR-124 overexpression could regulate $N R 4 A 1$ directly through its 3' UTR. This was able to cause a decrease in NR4A1 target genes and subsequent reduction in cell proliferation and spheroid growth of medulloblastoma cells. miR-124 is also reported to target AR to suppress the proliferation of prostate cancer [274], and miR-124 can target other pro-oncogenic genes [275, 276]. Another miRNA, miR-132, has been characterized as a regulator of $N R 4 A 2$ [239], although this relationship has not been studied in the context of cancer.

Due to the lack of understanding regarding miRNA regulation of NR4A receptors, we chose to investigate possible mechanisms in which dysregulation of a miRNA might lead to upregulation of $N R 4 A 2$ in cancer. We hypothesized for both $N R 4 A 1$ and $N R 4 A 2$, that putative miRNA regulators of these genes would be characterized as tumorsuppressor-like miRNAs. Tumor suppressor-like miRNAs are those that usually target a gene that is involved in promoting tumorigenesis, and the loss of this tumorsuppressor-like miRNA leads to an enhancement of oncogene expression. miR-124 and miR-132 have both been reported to contain hypermethylation in their promoters [277, 278], leading to suppression of these miRNAs in cancer, which could in part explain the observed upregulation of $N R 4 A 1$ and $N R 4 A 2$ in cancers, respectively. This mechanism of miRNA silencing is one common method in which tumors lose their expression of tumorsuppressor-like miRNAs [72].

Using a 3' UTR reporter assay, we screened a set of miRNAs that have been reported to be involved in cancer, including both oncomiRs and tumor-suppressor-like miRNAs. From this screen, our second ranked miRNA hit was miR-34, which is a welldescribed p53-regulated miRNA [70, 71]. Due to its direct transcriptional regulation by p53, miR-34 is described as a tumor-suppressor-like miRNA, as it targets many genes involved in cell proliferation, migration and metastasis, and suppression of apoptosis 
[75]. Upon p53 activation, miR-34 expression is increased along with other p53 target genes [71]. Due to the known involvement of NR4A2 in regulating p53 activity [95], we decided to further investigate the miR-34-NR4A2 relationship to determine if a p53miR-34-NR4A2 network existed.

Mutagenesis studies confirmed that miR-34 did indeed regulate NR4A2 through its 3' UTR. Overexpression of exogenous miR-34 was able to decrease NR4A2 at both gene and protein levels, confirming the regulation of endogenous NR4A2 expression. The effects of miR-34 were also confirmed to increase the p53 activity, as indicated by increased levels of acetylated p53 and target gene p21. This was likely through the observed downregulation of the deacetylase Sirtl by miR-34 [235], which is a negative regulator of $\mathrm{p} 53$ activity.

To determine if the regulation of $N R 4 A 2$ by miR-34 had biological relevance, our next step was to determine their endogenous relationship. To do so, we used a chemical activator of p53, Nutlin-3a [247], to induce the endogenous levels of miR-34. To determine the p53-mediated effect, we incorporated isogenic cell line pairs which either had wild-type p53 or deletion of p53. Using this method, we found that Nutlin-3a could suppress NR4A2 expression at the gene and protein levels in cells with wild-type p53, but not in the absence of p53. Future studies will aim to determine if the Nutlin$3 a-$ induced attenuation of NR4A2 expression is solely due to miR-34 following p53 activation and not because of other off-target effects. To do this, we will utilize a miR-34 inhibitor or antagomiR to block the endogenously-induced miR-34 during Nutlin-3a treatment. Alternatively, we can utilize genetic knockout models [234] or CRISPR-Cas9 genome editing to determine the NR4A2 levels in response to p53 activation in the absence of miR-34.

In the context of tumorigenesis, we used publically available data to determine if the miR-34-NR4A2 relationship was evident based on expression correlations. While one might expect correlations to exist between a miRNA and its target genes, this does not always hold true, as the miRNA-mRNA regulatory networks are quite complex [250]. Also, in CoMeTa analyses [245], NR4A2 did not score as a top hit for miR-34 targets, so we did not expect a robust negative correlation of miR-34 and NR4A2. Using a rectum carcinoma data set from The Cancer Genome Atlas (TGCA), we found a weak, inverse correlation of miR-34 with NR4A2, and this negative correlation was similar to the correlations found between miR-34 and its other published target genes.

Future studies in the lab will involve investigating other miRNAs of interest from our initial 3' UTR reporter screen. One miRNA in particular is miR-214, which was also highly ranked in our list of putative miRNAs. miR-214 has been reported to have varying roles in cancer depending on tissue type [279]. An area of interest is the regulation of miR-214 during myogenic differentiation [280, 281]. The dysregulation of muscle differentiation is believed to be a key mechanism of rhabdomyosarcoma tumor development and efforts are being put forth to target differentiation as a way to force cells into a non-proliferative state [282]. As we have also shown through our knockdown studies of NRAA2, there could be importance in studying the relationship of miR-214 and 
$N R 4 A 2$ in rhabdomyosarcoma, as miR-214 has been reported to be downregulated in rhabdomyosarcoma [283]. NR4A1 is also predicted to be a target of miR-214. miR-206, which is a well-described 'myo-miR' [284, 285] is also predicted to target several NR4A members. Studies are currently underway to understand the implications of the NR4A family in muscle differentiation and rhabdomyosarcoma development and progression, and to determine if the dynamics of the miR-206/214-NR4A relationships are important in these processes.

\section{NR4A Function in Cancer}

Knockdown and overexpression studies have demonstrated the importance of NR4A1 and NR4A2 in promoting cell proliferation, transformation, migration, and survival. This effect has been attributed to NR4A1 regulation of specific target genes [16, $47,48,85$ ], and several groups have aimed at targeting NR4A1 with small molecules [4755 ] to alter the expression of its critical downstream mediators of oncogenesis. The target genes of NR4A2 in cancer have not been well characterized, but NR4A2 has been associated with chemoresistance [93, 230, 231], possibly through its inhibition of p53 [95].

\section{NR4A2 and p53 suppression}

Corresponding to the previous identification of NR4A2-mediated suppression of p53 activity in response to doxorubicin [95], we found that NR4A2 could also suppress Nutlin-3a-induced p53 activity. Overexpression of NR4A2 led to an attenuation of p53 target gene $\mathrm{p} 21$, while knockdown of NR4A2 further enhanced the Nutlin-3a effect, suggesting an overall suppressive function of NR4A2. Using chromatin immunoprecipitation (ChIP), we were not able to clearly see an effect of p53 recruitment to target gene promoters in response to NR4A2 overexpression. Since NR4A2 expression is higher in cancer cells, another strategy that we could take is to perform the ChIP during knockdown of NR4A2 to determine if this can enhance the p53 association with gene promoters, either in the absence or presence of Nutlin-3a treatment. We also observed a reduction in both the p53 gene and protein levels in response to NR4A2 overexpression. Other mechanistic studies should be performed to clearly determine how NR4A2 is mediating its suppressive effect on p53 and its subsequent activation.

In terms of biological outcome, we found that overexpression of $N R 4 A 2$ could also rescue cells from Nutlin-3a-induced inhibition of cell proliferation. After prolonged treatment and observation, we determined that overexpression of $N R 4 A 2$ could suppress the $\mathrm{p} 53$ activity as indicated by reduced $\mathrm{p} 21$ and miR-34 expression. This model fits with the known involvement of NR4A2 with chemoresistance to 5-flurouracil [93, 230, 231], and more studies should be completed to determine the relationship of NR4A2 and miR34 in cell and mouse models of drug resistance. 
These findings hint to a possible feedback loop involving p53, miR-34, and NR4A2. Future studies related to this research could be to determine if NR4A2 can mediate effects in cancer depending on the p53 status. This would determine to what extent the effects of NR4A2 on chemoresistance, proliferation, and migration are mediated through $\mathrm{p} 53$. Additionally, an examination in patient tumor samples should be done to determine the correlation of NR4A2 expression as it relates to the p53 status and miR-34 expression.

\section{NR4A receptors in rhabdomyosarcoma}

Using cell models of rhabdomyosarcoma (RMS), we sought to understand the expression and function of the NR4A receptors. We found that the NR4A receptors generally had higher expression in the RMS cell lines when compared to normal muscle cells. Knockdown of $N R 4 A 1$ and $N R 4 A 2$ was able to reduce cell proliferation and clonogenicity of RMS cells, with $N R 4 A 2$ knockdown having the greater effect. Additionally, only NR4A2 was able to affect cell migration, which is supported by a previous finding of NR4A2 involvement in migration of bladder cancer cells [90].

To begin to understand how the NR4A receptors mediate their pro-oncogenic effects in the RMS cells, we performed a microarray study in which we knocked down the NR4A receptors in single, double, or triple combinations. Not surprisingly, the cells containing NR4A1 and NR4A3 knockdown tended to cluster together, while those with $N R 4 A 2$ had a distinct gene expression profile. Of the significantly altered genes, many of those altered by $N R 4 A 2$ knockdown were increased in expression, suggesting a possible role of NR4A2 in gene suppression which is reported for other nuclear receptors. A small cluster of genes were identified to be downregulated specifically by NR $4 A 2$ knockdown, and these genes will be validated for direct promoter regulation by NR4A2 to determine if they are true target genes.

The potential NR4A2-specific target genes from our microarray screen are of interest, and determining the importance of these genes as downstream mediators of NR4A2 activity could explain the role that NR4A2 has on cell migration in our experiments utilizing RMS, as none of the other NR4A members could affect this phenotypic response. It will also be critical to determine if NR4A2 can mediate its effects on proliferation and migration in a DNA-binding-dependent manner, which will require the use of DNA-binding mutants. This is probable due to fact that the RMS cell lines used contain mutations of p53 [286], so the effects of NR4A2 in these models is likely not due to the p53-NR4A2 relationship we have reported. Additionally, determining if upstream regulation of NR4As by miRNAs affects downstream transcriptional targets would give insights into coregulated gene expression cascades or networks. Understanding the effects of NR4A on these downstream mediators, either through miRNA regulation or small molecule inhibitors, can yield valuable information into potential uses as biomarkers or new biological targets. 


\section{LIST OF REFERENCES}

1. Govindan, M.V., et al., Cloning of the human glucocorticoid receptor cDNA. Nucleic Acids Res, 1985. 13(23): p. 8293-304.

2. Hollenberg, S.M., et al., Primary structure and expression of a functional human glucocorticoid receptor cDNA. Nature, 1985. 318(6047): p. 635-41.

3. Green, S., et al., Human oestrogen receptor cDNA: sequence, expression and homology to v-erb-A. Nature, 1986. 320(6058): p. 134-9.

4. Walter, P., et al., Cloning of the human estrogen receptor cDNA. Proc Natl Acad Sci U S A, 1985. 82(23): p. 7889-93.

5. Evans, R.M., The steroid and thyroid hormone receptor superfamily. Science, 1988. 240(4854): p. 889-95.

6. Giguere, V., et al., Functional domains of the human glucocorticoid receptor. Cell, 1986. 46(5): p. 645-52.

7. Kumar, V., et al., Functional domains of the human estrogen receptor. Cell, 1987. 51(6): p. 941-51.

8. $\quad$ Sladek, F.M., What are nuclear receptor ligands? Mol Cell Endocrinol, 2011. 334(1-2): p. 3-13.

9. Chen, T., Nuclear receptor drug discovery. Curr Opin Chem Biol, 2008. 12(4): p. 418-26.

10. Shi, Y., Orphan nuclear receptors in drug discovery. Drug Discov Today, 2007. 12(11-12): p. 440-5.

11. Overington, J.P., B. Al-Lazikani, and A.L. Hopkins, How many drug targets are there? Nat Rev Drug Discov, 2006. 5(12): p. 993-6.

12. Banerjee, M., D. Robbins, and T. Chen, Targeting xenobiotic receptors $P X R$ and CAR in human diseases. Drug Discov Today, 2015. 20(5): p. 618-28.

13. Willson, T.M. and S.A. Kliewer, PXR, CAR and drug metabolism. Nat Rev Drug Discov, 2002. 1(4): p. 259-66.

14. Warnmark, A., et al., Activation functions 1 and 2 of nuclear receptors: molecular strategies for transcriptional activation. Mol Endocrinol, 2003. 17(10): p. 1901-9.

15. Gronemeyer, H., J.A. Gustafsson, and V. Laudet, Principles for modulation of the nuclear receptor superfamily. Nat Rev Drug Discov, 2004. 3(11): p. 950-64.

16. Chen, H.Z., et al., Prolyl isomerase Pin1 stabilizes and activates orphan nuclear receptor TR3 to promote mitogenesis. Oncogene, 2012. 31(23): p. 2876-87.

17. Kato, S., et al., Activation of the estrogen receptor through phosphorylation by mitogen-activated protein kinase. Science, 1995. 270(5241): p. 1491-4.

18. Chen, W., et al., Glucocorticoid receptor phosphorylation differentially affects target gene expression. Mol Endocrinol, 2008. 22(8): p. 1754-66.

19. Helsen, C., et al., Structural basis for nuclear hormone receptor DNA binding. Mol Cell Endocrinol, 2012. 348(2): p. 411-7.

20. Picard, D. and K.R. Yamamoto, Two signals mediate hormone-dependent nuclear localization of the glucocorticoid receptor. EMBO J, 1987. 6(11): p. 3333-40.

21. Perlmann, T., et al., Two distinct dimerization interfaces differentially modulate target gene specificity of nuclear hormone receptors. Mol Endocrinol, 1996. 10(8): p. 958-66. 
22. Wurtz, J.M., et al., A canonical structure for the ligand-binding domain of nuclear receptors. Nat Struct Biol, 1996. 3(1): p. 87-94.

23. Renaud, J.P., et al., Crystal structure of the RAR-gamma ligand-binding domain bound to all-trans retinoic acid. Nature, 1995. 378(6558): p. 681-9.

24. Baschant, U., N.E. Lane, and J. Tuckermann, The multiple facets of glucocorticoid action in rheumatoid arthritis. Nat Rev Rheumatol, 2012. 8(11): p. 645-55.

25. Kadmiel, M. and J.A. Cidlowski, Glucocorticoid receptor signaling in health and disease. Trends Pharmacol Sci, 2013. 34(9): p. 518-30.

26. Pui, C.H. and W.E. Evans, Treatment of acute lymphoblastic leukemia. N Engl J Med, 2006. 354(2): p. 166-78.

27. Schlossmacher, G., A. Stevens, and A. White, Glucocorticoid receptor-mediated apoptosis: mechanisms of resistance in cancer cells. J Endocrinol, 2011. 211(1): p. 17-25.

28. Pui, C.H., et al., Impact of treatment efficacy on the prognostic value of glucocorticoid receptor levels in childhood acute lymphoblastic leukemia. Leuk Res, 1984. 8(3): p. 345-50.

29. Hillmann, A.G., et al., Glucocorticoid receptor gene mutations in leukemic cells acquired in vitro and in vivo. Cancer Res, 2000. 60(7): p. 2056-62.

30. Irving, J.A., et al., Loss of heterozygosity and somatic mutations of the glucocorticoid receptor gene are rarely found at relapse in pediatric acute lymphoblastic leukemia but may occur in a subpopulation early in the disease course. Cancer Res, 2005. 65(21): p. 9712-8.

31. Paugh, S.W., et al., NALP3 inflammasome upregulation and CASP1 cleavage of the glucocorticoid receptor cause glucocorticoid resistance in leukemia cells. Nat Genet, 2015. 47(6): p. 607-14.

32. Liang, J. and Y. Shang, Estrogen and cancer. Annu Rev Physiol, 2013. 75: p. 225-40.

33. Dubik, D. and R.P. Shiu, Mechanism of estrogen activation of c-myc oncogene expression. Oncogene, 1992. 7(8): p. 1587-94.

34. Sabbah, M., et al., Estrogen induction of the cyclin D1 promoter: involvement of a cAMP response-like element. Proc Natl Acad Sci U S A, 1999. 96(20): p. 11217 22.

35. Perillo, B., et al., 17beta-estradiol inhibits apoptosis in MCF-7 cells, inducing bcl-2 expression via two estrogen-responsive elements present in the coding sequence. Mol Cell Biol, 2000. 20(8): p. 2890-901.

36. Bendrik, C. and C. Dabrosin, Estradiol increases IL-8 secretion of normal human breast tissue and breast cancer in vivo. J Immunol, 2009. 182(1): p. 371-8.

37. Putti, T.C., et al., Estrogen receptor-negative breast carcinomas: a review of morphology and immunophenotypical analysis. Mod Pathol, 2005. 18(1): p. 2635.

38. Riggs, B.L. and L.C. Hartmann, Selective estrogen-receptor modulators -mechanisms of action and application to clinical practice. N Engl J Med, 2003. 348(7): p. 618-29.

39. Hoskins, J.M., L.A. Carey, and H.L. McLeod, CYP2D6 and tamoxifen: DNA matters in breast cancer. Nat Rev Cancer, 2009. 9(8): p. 576-86. 
40. Tan, M.H., et al., Androgen receptor: structure, role in prostate cancer and drug discovery. Acta Pharmacol Sin, 2015. 36(1): p. 3-23.

41. Cleutjens, K.B., et al., Two androgen response regions cooperate in steroid hormone regulated activity of the prostate-specific antigen promoter. J Biol Chem, 1996. 271(11): p. 6379-88.

42. Gottlieb, B., et al., The androgen receptor gene mutations database: 2012 update. Hum Mutat, 2012. 33(5): p. 887-94.

43. Karantanos, T., P.G. Corn, and T.C. Thompson, Prostate cancer progression after androgen deprivation therapy: mechanisms of castrate resistance and novel therapeutic approaches. Oncogene, 2013. 32(49): p. 5501-11.

44. Baek, S.H. and K.I. Kim, Emerging roles of orphan nuclear receptors in cancer. Annu Rev Physiol, 2014. 76: p. 177-95.

45. Beard, J.A., A. Tenga, and T. Chen, The interplay of NR4A receptors and the oncogene-tumor suppressor networks in cancer. Cell Signal, 2015. 27(2): p. 25766.

46. Mullican, S.E., et al., Abrogation of nuclear receptors Nr4a3 and Nr4al leads to development of acute myeloid leukemia. Nat Med, 2007. 13(6): p. 730-5.

47. Lee, S.O., et al., Inactivation of the orphan nuclear receptor TR3/Nur77 inhibits pancreatic cancer cell and tumor growth. Cancer Res, 2010. 70(17): p. 6824-36.

48. Lee, S.O., et al., The orphan nuclear receptor NR4A1 (Nur77) regulates oxidative and endoplasmic reticulum stress in pancreatic cancer cells. Mol Cancer Res, 2014. 12(4): p. 527-38.

49. Chintharlapalli, S., et al., Activation of Nur77 by selected 1,1-Bis(3'-indolyl)-1-(psubstituted phenyl)methanes induces apoptosis through nuclear pathways. J Biol Chem, 2005. 280(26): p. 24903-14.

50. Cho, S.D., et al., Activation of nerve growth factor-induced B alpha by methylenesubstituted diindolylmethanes in bladder cancer cells induces apoptosis and inhibits tumor growth. Mol Pharmacol, 2010. 77(3): p. 396-404.

51. Cho, S.D., et al., Nur77 agonists induce proapoptotic genes and responses in colon cancer cells through nuclear receptor-dependent and nuclear receptorindependent pathways. Cancer Res, 2007. 67(2): p. 674-83.

52. Lee, S.O., et al., p21 expression is induced by activation of nuclear nerve growth factor-induced Balpha (Nur77) in pancreatic cancer cells. Mol Cancer Res, 2009. 7(7): p. 1169-78.

53. Yoon, K., et al., Activation of nuclear TR3 (NR4A1) by a diindolylmethane analog induces apoptosis and proapoptotic genes in pancreatic cancer cells and tumors. Carcinogenesis, 2011. 32(6): p. 836-42.

54. Liu, J.J., et al., A unique pharmacophore for activation of the nuclear orphan receptor Nur77 in vivo and in vitro. Cancer Res, 2010. 70(9): p. 3628-37.

55. Zhan, Y., et al., Cytosporone B is an agonist for nuclear orphan receptor Nur 77. Nat Chem Biol, 2008. 4(9): p. 548-56.

56. Zhang, X.K., Targeting Nur77 translocation. Expert Opin Ther Targets, 2007. 11(1): p. 69-79.

57. Wang, W.J., et al., Orphan nuclear receptor TR3 acts in autophagic cell death via mitochondrial signaling pathway. Nat Chem Biol, 2014. 10(2): p. 133-40. 
58. Li, L., et al., Impeding the interaction between Nur77 and p38 reduces LPSinduced inflammation. Nat Chem Biol, 2015. 11(5): p. 339-46.

59. Zhan, Y.Y., et al., The orphan nuclear receptor Nur77 regulates LKB1 localization and activates AMPK. Nat Chem Biol, 2012 . 8(11): p. 897-904.

60. Lee, Y., et al., MicroRNA genes are transcribed by RNA polymerase II. EMBO J, 2004. 23(20): p. 4051-60.

61. Lee, Y., et al., The nuclear RNase III Drosha initiates microRNA processing. Nature, 2003. 425(6956): p. 415-9.

62. Yi, R., et al., Exportin-5 mediates the nuclear export of pre-microRNAs and short hairpin RNAs. Genes Dev, 2003. 17(24): p. 3011-6.

63. Hutvagner, G., et al., A cellular function for the RNA-interference enzyme Dicer in the maturation of the let-7 small temporal RNA. Science, 2001. 293(5531): $\mathrm{p}$. 834-8.

64. Kwak, P.B. and Y. Tomari, The $N$ domain of Argonaute drives duplex unwinding during RISC assembly. Nat Struct Mol Biol, 2012. 19(2): p. 145-51.

65. Huntzinger, E. and E. Izaurralde, Gene silencing by microRNAs: contributions of translational repression and $m R N A$ decay. Nat Rev Genet, 2011. 12(2): p. 99110.

66. Pasquinelli, A.E., MicroRNAs and their targets: recognition, regulation and an emerging reciprocal relationship. Nat Rev Genet, 2012. 13(4): p. 271-82.

67. Bartel, D.P., MicroRNAs: target recognition and regulatory functions. Cell, 2009. 136(2): p. 215-33.

68. Jansson, M.D. and A.H. Lund, MicroRNA and cancer. Mol Oncol, 2012. 6(6): p. 590-610.

69. Kent, O.A. and J.T. Mendell, A small piece in the cancer puzzle: microRNAs as tumor suppressors and oncogenes. Oncogene, 2006. 25(46): p. 6188-96.

70. He, L., et al., A microRNA component of the p53 tumour suppressor network. Nature, 2007. 447(7148): p. 1130-4.

71. Raver-Shapira, N., et al., Transcriptional activation of miR-34a contributes to p53-mediated apoptosis. Mol Cell, 2007. 26(5): p. 731-43.

72. Lopez-Serra, P. and M. Esteller, DNA methylation-associated silencing of tumorsuppressor microRNAs in cancer. Oncogene, 2012. 31(13): p. 1609-22.

73. Cole, K.A., et al., A functional screen identifies miR-34a as a candidate neuroblastoma tumor suppressor gene. Mol Cancer Res, 2008. 6(5): p. 735-42.

74. Feinberg-Gorenshtein, G., et al., Reduced levels of miR-34a in neuroblastoma are not caused by mutations in the TP53 binding site. Genes Chromosomes Cancer, 2009. 48(7): p. 539-43.

75. Hermeking, H., The miR-34 family in cancer and apoptosis. Cell Death Differ, 2010. 17(2): p. 193-9.

76. Chan, J.A., A.M. Krichevsky, and K.S. Kosik, MicroRNA-21 is an antiapoptotic factor in human glioblastoma cells. Cancer Res, 2005. 65(14): p. 6029-33.

77. Meng, F., et al., MicroRNA-21 regulates expression of the PTEN tumor suppressor gene in human hepatocellular cancer. Gastroenterology, 2007. 133(2): p. 647-58.

78. Maxwell, M.A. and G.E. Muscat, The NR4A subgroup: immediate early response genes with pleiotropic physiological roles. Nucl Recept Signal, 2006. 4: p. e002. 
79. Pearen, M.A. and G.E. Muscat, Minireview: Nuclear hormone receptor $4 \mathrm{~A}$ signaling: implications for metabolic disease. Mol Endocrinol, 2010. 24(10): p. 1891-903.

80. Baker, K.D., et al., The Drosophila orphan nuclear receptor DHR38 mediates an atypical ecdysteroid signaling pathway. Cell, 2003. 113(6): p. 731-42.

81. Flaig, R., et al., Structural basis for the cell-specific activities of the NGFI-B and the Nurrl ligand-binding domain. J Biol Chem, 2005. 280(19): p. 19250-8.

82. Wang, Z., et al., Structure and function of Nurr 1 identifies a class of ligandindependent nuclear receptors. Nature, 2003. 423(6939): p. 555-60.

83. Vinayavekhin, N. and A. Saghatelian, Discovery of a protein-metabolite interaction between unsaturated fatty acids and the nuclear receptor Nur77 using a metabolomics approach. J Am Chem Soc, 2011. 133(43): p. 17168-71.

84. Mohan, H.M., et al., Molecular pathways: the role of NR4A orphan nuclear receptors in cancer. Clinical cancer research : an official journal of the American Association for Cancer Research, 2012. 18(12): p. 3223-8.

85. $\mathrm{Mu}, \mathrm{X}$. and $\mathrm{C}$. Chang, TR3 orphan nuclear receptor mediates apoptosis through up-regulating E2F1 in human prostate cancer LNCaP cells. J Biol Chem, 2003. 278(44): p. 42840-5.

86. Safe, S., et al., NR4A orphan receptors and cancer. Nucl Recept Signal, 2011. 9: p. e002.

87. Lee, S.O., et al., Targeting NR4A1 (TR3) in cancer cells and tumors. Expert Opin Ther Targets, 2011. 15(2): p. 195-206.

88. Ke, N., et al., Nuclear hormone receptor NR4A2 is involved in cell transformation and apoptosis. Cancer Res, 2004. 64(22): p. 8208-12.

89. Komiya, T., et al., Enhanced activity of the CREB co-activator Crtcl in LKB1 null lung cancer. Oncogene, 2010. 29(11): p. 1672-80.

90. Inamoto, T., et al., Cytoplasmic mislocalization of the orphan nuclear receptor Nurr1 is a prognostic factor in bladder cancer. Cancer, 2010. 116(2): p. 340-6.

91. Li, X. and H.H. Tai, Activation of thromboxane A(2) receptors induces orphan nuclear receptor Nurrl expression and stimulates cell proliferation in human lung cancer cells. Carcinogenesis, 2009. 30(9): p. 1606-13.

92. Llopis, S., et al., Dichotomous roles for the orphan nuclear receptor NURR1 in breast cancer. BMC Cancer, 2013. 13: p. 139.

93. Shigeishi, H., et al., PGE(2) targets squamous cell carcinoma cell with the activated epidermal growth factor receptor family for survival against 5fluorouracil through NR4A2 induction. Cancer Lett, 2011. 307(2): p. 227-36.

94. Holla, V.R., et al., Prostaglandin E2 regulates the nuclear receptor NR4A2 in colorectal cancer. J Biol Chem, 2006. 281(5): p. 2676-82.

95. Zhang, T., et al., NGFI-B nuclear orphan receptor Nurrl interacts with $p 53$ and suppresses its transcriptional activity. Mol Cancer Res, 2009. 7(8): p. 1408-15.

96. Lee, S.L., et al., Unimpaired thymic and peripheral T cell death in mice lacking the nuclear receptor NGFI-B (Nur77). Science, 1995. 269(5223): p. 532-5.

97. Ponnio, T., et al., The nuclear receptor Nor-1 is essential for proliferation of the semicircular canals of the mouse inner ear. Mol Cell Biol, 2002. 22(3): p. 935-45. 
98. Boudreaux, S.P., et al., Genome-wide profiling reveals transcriptional repression of MYC as a core component of NR4A tumor suppression in acute myeloid leukemia. Oncogenesis, 2012. 1: p. e19.

99. Labelle, Y., et al., Oncogenic conversion of a novel orphan nuclear receptor by chromosome translocation. Hum Mol Genet, 1995. 4(12): p. 2219-26.

100. Labelle, Y., et al., The EWS/TEC fusion protein encoded by the $t(9 ; 22)$ chromosomal translocation in human chondrosarcomas is a highly potent transcriptional activator. Oncogene, 1999. 18(21): p. 3303-8.

101. Filion, C. and Y. Labelle, Identification of genes regulated by the EWS/NR4A3 fusion protein in extraskeletal myxoid chondrosarcoma. Tumour Biol, 2012. 33(5): p. 1599-605.

102. Ohkura, N., Y. Nagamura, and T. Tsukada, Differential transactivation by orphan nuclear receptor NOR1 and its fusion gene product EWS/NOR1: possible involvement of poly(ADP-ribose) polymerase I, PARP-1. J Cell Biochem, 2008. 105(3): p. 785-800.

103. Panagopoulos, I., et al., Molecular genetic characterization of the EWS/CHN and RBP56/CHN fusion genes in extraskeletal myxoid chondrosarcoma. Genes Chromosomes Cancer, 2002. 35(4): p. 340-52.

104. Filion, C. and Y. Labelle, The oncogenic fusion protein EWS/NOR-1 induces transformation of CFK2 chondrogenic cells. Exp Cell Res, 2004. 297(2): p. 58592.

105. Sjogren, H., et al., Fusion of the EWS-related gene TAF2N to TEC in extraskeletal myxoid chondrosarcoma. Cancer Res, 1999. 59(20): p. 5064-7.

106. Sjogren, H., et al., Fusion of the NH2-terminal domain of the basic helix-loophelix protein TCF12 to TEC in extraskeletal myxoid chondrosarcoma with translocation t(9;15)(q22;q21). Cancer Res, 2000. 60(24): p. 6832-5.

107. Dhillon, A.S., et al., MAP kinase signalling pathways in cancer. Oncogene, 2007. 26(22): p. 3279-90.

108. Slagsvold, H.H., et al., Nuclear receptor and apoptosis initiator NGFI-B is a substrate for kinase ERK2. Biochem Biophys Res Commun, 2002. 291(5): p. 1146-50.

109. Strom, B.O. and R.E. Paulsen, Apoptosis inducer NGFI-B is degraded by the proteasome and stabilized by treatment with EGF. Biochem Biophys Res Commun, 2012. 417(4): p. 1292-7.

110. Chen, H.Z., et al., Prolyl isomerase Pin1 stabilizes and activates orphan nuclear receptor TR3 to promote mitogenesis. Oncogene, 2011.

111. Jacobs, C.M., et al., ERK2 prohibits apoptosis-induced subcellular translocation of orphan nuclear receptor NGFI-B/TR3. J Biol Chem, 2004. 279(48): p. 50097101.

112. van den Brink, M.R., et al., The extracellular signal-regulated kinase pathway is required for activation-induced cell death of T cells. J Biol Chem, 1999. 274(16): p. $11178-85$.

113. Wang, A., et al., Phosphorylation of Nur77 by the MEK-ERK-RSK cascade induces mitochondrial translocation and apoptosis in T cells. J Immunol, 2009. 183(5): p. 3268-77. 
114. Wingate, A.D., et al., Nur77 is phosphorylated in cells by RSK in response to mitogenic stimulation. Biochem J, 2006. 393(Pt 3): p. 715-24.

115. Teixeiro, E. and M.A. Daniels, ERK and cell death: ERK location and T cell selection. FEBS J, 2010. 277(1): p. 30-8.

116. Fujii, Y., et al., ERK5 is involved in TCR-induced apoptosis through the modification of Nur77. Genes Cells, 2008. 13(5): p. 411-9.

117. Yang, H., et al., Induction and intracellular localization of Nur77 dictate fenretinide-induced apoptosis of human liver cancer cells. Biochem Pharmacol, 2010. 79(7): p. 948-54.

118. Smith, A.G., et al., Regulation of NR4A nuclear receptor expression by oncogenic $B R A F$ in melanoma cells. Pigment Cell Melanoma Res, 2011. 24(3): p. 551-63.

119. Sekine, Y., et al., p38 MAPKs regulate the expression of genes in the dopamine synthesis pathway through phosphorylation of NR4A nuclear receptors. J Cell Sci, 2011. 124(Pt 17): p. 3006-16.

120. Lammi, J. and P. Aarnisalo, FGF-8 stimulates the expression of NR4A orphan nuclear receptors in osteoblasts. Mol Cell Endocrinol, 2008. 295(1-2): p. 87-93.

121. Vivanco, I. and C.L. Sawyers, The phosphatidylinositol 3-Kinase AKT pathway in human cancer. Nat Rev Cancer, 2002. 2(7): p. 489-501.

122. Whitman, M., et al., Type I phosphatidylinositol kinase makes a novel inositol phospholipid, phosphatidylinositol-3-phosphate. Nature, 1988. 332(6165): p. 6446.

123. Alessi, D.R., et al., Characterization of a 3-phosphoinositide-dependent protein kinase which phosphorylates and activates protein kinase Balpha. Curr Biol, 1997. 7(4): p. 261-9.

124. Stephens, L., et al., Protein kinase B kinases that mediate phosphatidylinositol 3,4,5-trisphosphate-dependent activation of protein kinase B. Science, 1998. 279(5351): p. 710-4.

125. Maehama, T. and J.E. Dixon, The tumor suppressor, PTEN/MMAC1, dephosphorylates the lipid second messenger, phosphatidylinositol 3,4,5trisphosphate. J Biol Chem, 1998. 273(22): p. 13375-8.

126. Stambolic, V., et al., Negative regulation of PKB/Akt-dependent cell survival by the tumor suppressor PTEN. Cell, 1998. 95(1): p. 29-39.

127. Datta, S.R., et al., Akt phosphorylation of BAD couples survival signals to the cell-intrinsic death machinery. Cell, 1997. 91(2): p. 231-41.

128. Cardone, M.H., et al., Regulation of cell death protease caspase-9 by phosphorylation. Science, 1998. 282(5392): p. 1318-21.

129. Diehl, J.A., et al., Glycogen synthase kinase-3beta regulates cyclin D1 proteolysis and subcellular localization. Genes Dev, 1998. 12(22): p. 3499-511.

130. Porta, C., C. Paglino, and A. Mosca, Targeting PI3K/Akt/mTOR Signaling in Cancer. Front Oncol, 2014. 4: p. 64.

131. Engelman, J.A., J. Luo, and L.C. Cantley, The evolution of phosphatidylinositol 3kinases as regulators of growth and metabolism. Nat Rev Genet, 2006. 7(8): p. 606-19.

132. Chalhoub, N. and S.J. Baker, PTEN and the PI3-kinase pathway in cancer. Annu Rev Pathol, 2009. 4: p. 127-50. 
133. Li, J., et al., PTEN, a putative protein tyrosine phosphatase gene mutated in human brain, breast, and prostate cancer. Science, 1997. 275(5308): p. 1943-7.

134. Steck, P.A., et al., Identification of a candidate tumour suppressor gene, MMACl, at chromosome 10q23.3 that is mutated in multiple advanced cancers. Nat Genet, 1997. 15(4): p. 356-62.

135. Di Cristofano, A., et al., Pten is essential for embryonic development and tumour suppression. Nat Genet, 1998. 19(4): p. 348-55.

136. Mayo, L.D. and D.B. Donner, A phosphatidylinositol 3-kinase/Akt pathway promotes translocation of Mdm2 from the cytoplasm to the nucleus. Proc Natl Acad Sci U S A, 2001. 98(20): p. 11598-603.

137. Chen, H.Z., et al., Akt phosphorylates the TR3 orphan receptor and blocks its targeting to the mitochondria. Carcinogenesis, 2008. 29(11): p. 2078-88.

138. Masuyama, N., et al., Akt inhibits the orphan nuclear receptor Nur77 and T-cell apoptosis. J Biol Chem, 2001. 276(35): p. 32799-805.

139. Pekarsky, Y., et al., Akt phosphorylates and regulates the orphan nuclear receptor Nur77. Proc Natl Acad Sci U S A, 2001. 98(7): p. 3690-4.

140. Hirata, Y., et al., The phosphorylation and DNA binding of the DNA-binding domain of the orphan nuclear receptor NGFI-B. J Biol Chem, 1993. 268(33): $\mathrm{p}$. 24808-12.

141. Li, H., et al., Cytochrome c release and apoptosis induced by mitochondrial targeting of nuclear orphan receptor TR3. Science, 2000. 289(5482): p. 1159-64.

142. Han, Y.H., et al., Regulation of Nur77 nuclear export by c-Jun N-terminal kinase and Akt. Oncogene, 2006. 25(21): p. 2974-86.

143. Wilson, A.J., et al., TR3 modulates platinum resistance in ovarian cancer. Cancer Res, 2013. 73(15): p. 4758-69.

144. Gao, X., et al., Tsc tumour suppressor proteins antagonize amino-acid-TOR signalling. Nat Cell Biol, 2002. 4(9): p. 699-704.

145. Potter, C.J., L.G. Pedraza, and T. Xu, Akt regulates growth by directly phosphorylating Tsc2. Nat Cell Biol, 2002. 4(9): p. 658-65.

146. Lizcano, J.M., et al., LKB1 is a master kinase that activates 13 kinases of the AMPK subfamily, including MARK/PAR-1. EMBO J, 2004. 23(4): p. 833-43.

147. Shaw, R.J., et al., The LKB1 tumor suppressor negatively regulates $m T O R$ signaling. Cancer Cell, 2004. 6(1): p. 91-9.

148. Lee, S.O., et al., The nuclear receptor TR 3 regulates $m$ TORC1 signaling in lung cancer cells expressing wild-type p53. Oncogene, 2012. 31(27): p. 3265-76.

149. Budanov, A.V. and M. Karin, $p 53$ target genes sestrin1 and sestrin2 connect genotoxic stress and mTOR signaling. Cell, 2008. 134(3): p. 451-60.

150. Zhao, B.X., et al., p53 mediates the negative regulation of MDM2 by orphan receptor TR3. EMBO J, 2006. 25(24): p. 5703-15.

151. Semenza, G.L., Hypoxia-inducible factors in physiology and medicine. Cell, 2012. 148(3): p. 399-408.

152. Wang, G.L., et al., Hypoxia-inducible factor 1 is a basic-helix-loop-helix-PAS heterodimer regulated by cellular O2 tension. Proc Natl Acad Sci U S A, 1995. 92(12): p. 5510-4.

153. Wang, G.L. and G.L. Semenza, Purification and characterization of hypoxiainducible factor 1. J Biol Chem, 1995. 270(3): p. 1230-7. 
154. Ivan, M., et al., HIFalpha targeted for VHL-mediated destruction by proline hydroxylation: implications for O2 sensing. Science, 2001. 292(5516): p. 464-8.

155. Jaakkola, P., et al., Targeting of HIF-alpha to the von Hippel-Lindau ubiquitylation complex by O2-regulated prolyl hydroxylation. Science, 2001. 292(5516): p. 468-72.

156. Maxwell, P.H., et al., The tumour suppressor protein VHL targets hypoxiainducible factors for oxygen-dependent proteolysis. Nature, 1999. 399(6733): p. 271-5.

157. Ohh, M., et al., Ubiquitination of hypoxia-inducible factor requires direct binding to the beta-domain of the von Hippel-Lindau protein. Nat Cell Biol, 2000. 2(7): p. 423-7.

158. Lando, D., et al., FIH-1 is an asparaginyl hydroxylase enzyme that regulates the transcriptional activity of hypoxia-inducible factor. Genes Dev, 2002. 16(12): p. 1466-71.

159. Semenza, G.L. and G.L. Wang, A nuclear factor induced by hypoxia via de novo protein synthesis binds to the human erythropoietin gene enhancer at a site required for transcriptional activation. Mol Cell Biol, 1992. 12(12): p. 5447-54.

160. Forsythe, J.A., et al., Activation of vascular endothelial growth factor gene transcription by hypoxia-inducible factor 1. Mol Cell Biol, 1996. 16(9): p. 460413.

161. Chen, C., et al., Regulation of glut1 mRNA by hypoxia-inducible factor-1. Interaction between H-ras and hypoxia. J Biol Chem, 2001. 276(12): p. 9519-25.

162. Semenza, G.L., et al., Hypoxia response elements in the aldolase A, enolase 1, and lactate dehydrogenase A gene promoters contain essential binding sites for hypoxia-inducible factor 1. J Biol Chem, 1996. 271(51): p. 32529-37.

163. Kim, J.W., et al., HIF-1-mediated expression of pyruvate dehydrogenase kinase: a metabolic switch required for cellular adaptation to hypoxia. Cell Metab, 2006. 3(3): p. 177-85.

164. Papandreou, I., et al., HIF-1 mediates adaptation to hypoxia by actively downregulating mitochondrial oxygen consumption. Cell Metab, 2006. 3(3): p. 187-97.

165. Semenza, G.L., Defining the role of hypoxia-inducible factor 1 in cancer biology and therapeutics. Oncogene, 2010. 29(5): p. 625-34.

166. Weidemann, A. and R.S. Johnson, Biology of HIF-1alpha. Cell Death Differ, 2008. 15(4): p. 621-7.

167. Hanahan, D. and R.A. Weinberg, The hallmarks of cancer. Cell, 2000. 100(1): p. 57-70.

168. Talks, K.L., et al., The expression and distribution of the hypoxia-inducible factors HIF-1alpha and HIF-2alpha in normal human tissues, cancers, and tumor-associated macrophages. Am J Pathol, 2000. 157(2): p. 411-21.

169. Zhong, H., et al., Overexpression of hypoxia-inducible factor lalpha in common human cancers and their metastases. Cancer Res, 1999. 59(22): p. 5830-5.

170. Zagzag, D., et al., Expression of hypoxia-inducible factor lalpha in brain tumors: association with angiogenesis, invasion, and progression. Cancer, 2000. 88(11): p. 2606-18. 
171. Latif, F., et al., Identification of the von Hippel-Lindau disease tumor suppressor gene. Science, 1993. 260(5112): p. 1317-20.

172. Moore, L.E., et al., Von Hippel-Lindau (VHL) inactivation in sporadic clear cell renal cancer: associations with germline VHL polymorphisms and etiologic risk factors. PLoS Genet, 2011. 7(10): p. e1002312.

173. Choi, J.W., et al., Nur77 activated by hypoxia-inducible factor-1alpha overproduces proopiomelanocortin in von Hippel-Lindau-mutated renal cell carcinoma. Cancer Res, 2004. 64(1): p. 35-9.

174. Yoo, Y.G., et al., Novel function of orphan nuclear receptor Nur77 in stabilizing hypoxia-inducible factor-1alpha. J Biol Chem, 2004. 279(51): p. 53365-73.

175. Yoo, Y.G., et al., 6-Mercaptopurine, an activator of Nur77, enhances transcriptional activity of HIF-1alpha resulting in new vessel formation. Oncogene, 2007. 26(26): p. 3823-34.

176. Martorell, L., et al., The hypoxia-inducible factor 1/NOR-1 axis regulates the survival response of endothelial cells to hypoxia. Mol Cell Biol, 2009. 29(21): p. 5828-42.

177. To, S.K., et al., Hypoxia triggers a Nur77-beta-catenin feed-forward loop to promote the invasive growth of colon cancer cells. Br J Cancer, 2014. 110(4): p. 935-45.

178. Kim, B.Y., et al., Nur77 upregulates HIF-alpha by inhibiting $p V H L$-mediated degradation. Exp Mol Med, 2008. 40(1): p. 71-83.

179. Wansa, K.D., et al., The AF-1 domain of the orphan nuclear receptor NOR-1 mediates trans-activation, coactivator recruitment, and activation by the purine anti-metabolite 6-mercaptopurine. J Biol Chem, 2003. 278(27): p. 24776-90.

180. Wu, H., et al., Regulation of Nur77 expression by beta-catenin and its mitogenic effect in colon cancer cells. FASEB J, 2011. 25(1): p. 192-205.

181. Liu, D., et al., Vascular endothelial growth factor-regulated gene expression in endothelial cells: KDR-mediated induction of Egr3 and the related nuclear receptors Nur77, Nurr1, and Nor1. Arterioscler Thromb Vasc Biol, 2003. 23(11): p. 2002-7.

182. Rius, J., et al., NOR-1 is involved in VEGF-induced endothelial cell growth. Atherosclerosis, 2006. 184(2): p. 276-82.

183. Zeng, H., et al., Orphan nuclear receptor TR3/Nur77 regulates VEGF-A-induced angiogenesis through its transcriptional activity. J Exp Med, 2006. 203(3): p. 719-29.

184. Zhao, D., S. Desai, and H. Zeng, VEGF stimulates PKD-mediated CREBdependent orphan nuclear receptor Nurrl expression: role in VEGF-induced angiogenesis. Int J Cancer, 2011. 128(11): p. 2602-12.

185. Martinez-Gonzalez, J., et al., Neuron-derived orphan receptor-1 (NOR-1) modulates vascular smooth muscle cell proliferation. Circ Res, 2003. 92(1): p. 96-103.

186. Levine, A.J., p53, the cellular gatekeeper for growth and division. Cell, 1997. 88(3): p. 323-31.

187. Zilfou, J.T. and S.W. Lowe, Tumor suppressive functions of p53. Cold Spring Harb Perspect Biol, 2009. 1(5): p. a001883. 
188. el-Deiry, W.S., et al., WAF1/CIP1 is induced in p53-mediated G1 arrest and apoptosis. Cancer Res, 1994. 54(5): p. 1169-74.

189. el-Deiry, W.S., et al., WAF1, a potential mediator of p53 tumor suppression. Cell, 1993. 75(4): p. 817-25.

190. Kastan, M.B., et al., A mammalian cell cycle checkpoint pathway utilizing p53 and GADD45 is defective in ataxia-telangiectasia. Cell, 1992. 71(4): p. 587-97.

191. Nakano, K. and K.H. Vousden, PUMA, a novel proapoptotic gene, is induced by p53. Mol Cell, 2001. 7(3): p. 683-94.

192. Oda, E., et al., Noxa, a BH3-only member of the Bcl-2 family and candidate mediator of p53-induced apoptosis. Science, 2000. 288(5468): p. 1053-8.

193. Miyashita, T. and J.C. Reed, Tumor suppressor p53 is a direct transcriptional activator of the human bax gene. Cell, 1995. 80(2): p. 293-9.

194. Mihara, M., et al., p53 has a direct apoptogenic role at the mitochondria. Mol Cell, 2003. 11(3): p. 577-90.

195. Levine, A.J. and M. Oren, The first 30 years of p53: growing ever more complex. Nat Rev Cancer, 2009. 9(10): p. 749-58.

196. de Vries, A., et al., Targeted point mutations of 553 lead to dominant-negative inhibition of wild-type p53 function. Proc Natl Acad Sci U S A, 2002. 99(5): p. 2948-53.

197. Freed-Pastor, W.A. and C. Prives, Mutant p53: one name, many proteins. Genes Dev, 2012. 26(12): p. 1268-86.

198. Oliner, J.D., et al., Amplification of a gene encoding a p53-associated protein in human sarcomas. Nature, 1992. 358(6381): p. 80-3.

199. Jacks, T., et al., Tumor spectrum analysis in p53-mutant mice. Curr Biol, 1994. 4(1): p. 1-7.

200. Zhao, B.X., et al., Orphan receptor TR3 enhances $p 53$ transactivation and represses DNA double-strand break repair in hepatoma cells under ionizing radiation. Mol Endocrinol, 2011. 25(8): p. 1337-50.

201. Malewicz, M., et al., Essential role for DNA-PK-mediated phosphorylation of NR4A nuclear orphan receptors in DNA double-strand break repair. Genes Dev, 2011. 25(19): p. 2031-40.

202. Moll, U.M., N. Marchenko, and X.K. Zhang, p53 and Nur77/TR3 - transcription factors that directly target mitochondria for cell death induction. Oncogene, 2006. 25(34): p. 4725-43.

203. Wu, Q., et al., Dual roles of Nur77 in selective regulation of apoptosis and cell cycle by TPA and ATRA in gastric cancer cells. Carcinogenesis, 2002. 23(10): p. 1583-92.

204. Cao, X., et al., Retinoid X receptor regulates Nur77/TR3-dependent apoptosis by modulating its nuclear export and mitochondrial targeting. Mol Cell Biol, 2004. 24(22): p. 9705-25.

205. Katagiri, Y., et al., Modulation of retinoid signalling through NGF-induced nuclear export of NGFI-B. Nat Cell Biol, 2000. 2(7): p. 435-40.

206. Lin, X.F., et al., RXRalpha acts as a carrier for TR3 nuclear export in a 9-cis retinoic acid-dependent manner in gastric cancer cells. J Cell Sci, 2004. 117(Pt 23): p. 5609-21. 
207. Lin, B., et al., Conversion of Bcl-2 from protector to killer by interaction with nuclear orphan receptor Nur77/TR3. Cell, 2004. 116(4): p. 527-40.

208. Liang, B., et al., Involvement of TR3/Nur77 translocation to the endoplasmic reticulum in ER stress-induced apoptosis. Exp Cell Res, 2007. 313(13): p. 283344.

209. Inamoto, T., et al., 1,1-Bis(3'-indolyl)-1-(p-chlorophenyl)methane activates the orphan nuclear receptor Nurrl and inhibits bladder cancer growth. Mol Cancer Ther, 2008. 7(12): p. 3825-33.

210. Anastas, J.N. and R.T. Moon, WNT signalling pathways as therapeutic targets in cancer. Nat Rev Cancer, 2013. 13(1): p. 11-26.

211. Rao, T.P. and M. Kuhl, An updated overview on Wht signaling pathways: a prelude for more. Circulation research, 2010. 106(12): p. 1798-806.

212. Mulholland, D.J., et al., Interaction of nuclear receptors with the Wnt/betacatenin/Tcf signaling axis: Wnt you like to know? Endocr Rev, 2005. 26(7): p. 898-915.

213. Rajalin, A.M. and P. Aarnisalo, Cross-talk between NR4A orphan nuclear receptors and beta-catenin signaling pathway in osteoblasts. Archives of biochemistry and biophysics, 2011. 509(1): p. 44-51.

214. To, S.K., J.Z. Zeng, and A.S. Wong, Nur77: a potential therapeutic target in cancer. Expert Opin Ther Targets, 2012. 16(6): p. 573-85.

215. Flaus, A., et al., Identification of multiple distinct Snf2 subfamilies with conserved structural motifs. Nucleic Acids Res, 2006. 34(10): p. 2887-905.

216. Karras, G.I., et al., The macro domain is an ADP-ribose binding module. EMBO J, 2005. 24(11): p. 1911-20.

217. Ahel, D., et al., Poly(ADP-ribose)-dependent regulation of DNA repair by the chromatin remodeling enzyme ALC1. Science, 2009. 325(5945): p. 1240-3.

218. Gottschalk, A.J., et al., Poly(ADP-ribosyl)ation directs recruitment and activation of an ATP-dependent chromatin remodeler. Proc Natl Acad Sci U S A, 2009. 106(33): p. 13770-4.

219. Chen, L., et al., CHD1L promotes hepatocellular carcinoma progression and metastasis in mice and is associated with these processes in human patients. $\mathrm{J}$ Clin Invest, 2010. 120(4): p. 1178-91.

220. Chan, T.H., et al., Translationally controlled tumor protein induces mitotic defects and chromosome missegregation in hepatocellular carcinoma development. Hepatology, 2012. 55(2): p. 491-505.

221. Li, Y., et al., SPOCK1 is regulated by CHDIL and blocks apoptosis and promotes HCC cell invasiveness and metastasis in mice. Gastroenterology, 2013. 144(1): p. 179-191 e4.

222. Cheng, W., Y. Su, and F. Xu, CHDIL: a novel oncogene. Mol Cancer, 2013. 12(1): p. 170.

223. Chen, L., T.H. Chan, and X.Y. Guan, Chromosome 1q21 amplification and oncogenes in hepatocellular carcinoma. Acta Pharmacol Sin, 2010. 31(9): p. 1165-71.

224. Ma, N.F., et al., Isolation and characterization of a novel oncogene, amplified in liver cancer 1, within a commonly amplified region at 1q21 in hepatocellular carcinoma. Hepatology, 2008. 47(2): p. 503-10. 
225. Guan, X.Y., et al., Recurrent chromosome alterations in hepatocellular carcinoma detected by comparative genomic hybridization. Genes Chromosomes Cancer, 2000. 29(2): p. 110-6.

226. Chen, L., et al., Clinical significance of CHD1L in hepatocellular carcinoma and therapeutic potentials of virus-mediated CHD1L depletion. Gut, 2011. 60(4): p. 534-43.

227. Chen, M., et al., Transgenic CHDlL expression in mouse induces spontaneous tumors. PLoS One, 2009. 4(8): p. e6727.

228. Sahai, E. and C.J. Marshall, RHO-GTPases and cancer. Nat Rev Cancer, 2002. 2(2): p. 133-42.

229. Chen, L., et al., Chromodomain helicase/adenosine triphosphatase DNA binding protein 1-like (CHD1l) gene suppresses the nucleus-to-mitochondria translocation of nur77 to sustain hepatocellular carcinoma cell survival. Hepatology, 2009. 50(1): p. 122-9.

230. Han, Y., et al., Nuclear orphan receptor NR4A2 confers chemoresistance and predicts unfavorable prognosis of colorectal carcinoma patients who received postoperative chemotherapy. Eur J Cancer, 2013. 49(16): p. 3420-30.

231. Han, Y., et al., Expression of orphan nuclear receptor NR4A2 in gastric cancer cells confers chemoresistance and predicts an unfavorable postoperative survival of gastric cancer patients with chemotherapy. Cancer, 2013. 119(19): p. 3436-45.

232. Chang, T.C., et al., Transactivation of miR-34a by p53 broadly influences gene expression and promotes apoptosis. Mol Cell, 2007. 26(5): p. 745-52.

233. Misso, G., et al., Mir-34: a new weapon against cancer? Mol Ther Nucleic Acids, 2014. 3: p. e194.

234. Concepcion, C.P., et al., Intact p53-dependent responses in miR-34-deficient mice. PLoS Genet, 2012. 8(7): p. e1002797.

235. Yamakuchi, M., M. Ferlito, and C.J. Lowenstein, miR-34a repression of SIRT1 regulates apoptosis. Proc Natl Acad Sci U S A, 2008. 105(36): p. 13421-6.

236. Okada, N., et al., A positive feedback between $p 53$ and miR-34 miRNAs mediates tumor suppression. Genes Dev, 2014. 28(5): p. 438-50.

237. Lodygin, D., et al., Inactivation of miR-34a by aberrant $C p G$ methylation in multiple types of cancer. Cell Cycle, 2008. 7(16): p. 2591-600.

238. Wang, Z., et al., DNA hypermethylation of microR $A$ A-34b/c has prognostic value for stage non-small cell lung cancer. Cancer Biol Ther, 2011. 11(5): p. 490-6.

239. Yang, D., et al., miR-132 regulates the differentiation of dopamine neurons by directly targeting Nurr1 expression. J Cell Sci, 2012. 125(Pt 7): p. 1673-82.

240. Wu, S., et al., MicroRNA-132 promotes estradiol synthesis in ovarian granulosa cells via translational repression of Nurr1. Reprod Biol Endocrinol, 2015. 13(1): p. 94.

241. Takwi, A.A., et al., miR-137 regulates the constitutive androstane receptor and modulates doxorubicin sensitivity in parental and doxorubicin-resistant neuroblastoma cells. Oncogene, 2014. 33(28): p. 3717-29.

242. Lewis, B.P., C.B. Burge, and D.P. Bartel, Conserved seed pairing, often flanked by adenosines, indicates that thousands of human genes are microRNA targets. Cell, 2005. 120(1): p. 15-20.

243. John, B., et al., Human MicroRNA targets. PLoS Biol, 2004. 2(11): p. e363. 
244. Krek, A., et al., Combinatorial microRNA target predictions. Nat Genet, 2005. 37(5): p. 495-500.

245. Gennarino, V.A., et al., Identification of microRNA-regulated gene networks by expression analysis of target genes. Genome Res, 2012. 22(6): p. 1163-72.

246. Mudduluru, G., et al., Regulation of Axl receptor tyrosine kinase expression by miR-34a and miR-199a/b in solid cancer. Oncogene, 2011. 30(25): p. 2888-99.

247. Vassilev, L.T., et al., In vivo activation of the 553 pathway by small-molecule antagonists of MDM2. Science, 2004. 303(5659): p. 844-8.

248. Millau, J.F., et al., Formation of stress-specific p53 binding patterns is influenced by chromatin but not by modulation of 53 binding affinity to response elements. Nucleic Acids Res, 2011. 39(8): p. 3053-63.

249. Ohata, H., et al., NuMA is required for the selective induction of p53 target genes. Mol Cell Biol, 2013. 33(12): p. 2447-57.

250. Peter, M.E., Targeting of mRNAs by multiple miRNAs: the next step. Oncogene, 2010. 29(15): p. 2161-4.

251. Bader, A.G., miR-34 - a microRNA replacement therapy is headed to the clinic. Front Genet, 2012. 3: p. 120.

252. Li, Z. and T.M. Rana, Therapeutic targeting of microRNAs: current status and future challenges. Nat Rev Drug Discov, 2014. 13(8): p. 622-38.

253. Corney, D.C., et al., Frequent downregulation of miR-34 family in human ovarian cancers. Clin Cancer Res, 2010. 16(4): p. 1119-28.

254. Gallardo, E., et al., miR-34a as a prognostic marker of relapse in surgically resected non-small-cell lung cancer. Carcinogenesis, 2009. 30(11): p. 1903-9.

255. Nakatani, F., et al., miR-34a predicts survival of Ewing's sarcoma patients and directly influences cell chemo-sensitivity and malignancy. J Pathol, 2012. 226(5): p. 796-805.

256. Svoboda, M., et al., MiR-34b is associated with clinical outcome in triplenegative breast cancer patients. Diagn Pathol, 2012. 7: p. 31.

257. Wang, J., et al., The predictive effect of overexpressed miR-34a on good survival of cancer patients: a systematic review and meta-analysis. Onco Targets Ther, 2015. 8: p. 2709-19.

258. Zhao, J., K. Kelnar, and A.G. Bader, In-depth analysis shows synergy between erlotinib and miR-34a. PLoS One, 2014. 9(2): p. e89105.

259. Li, X.J., et al., MicroRNA-34a modulates chemosensitivity of breast cancer cells to adriamycin by targeting Notch1. Arch Med Res, 2012. 43(7): p. 514-21.

260. Akao, Y., et al., Dysregulation of microRNA-34a expression causes drugresistance to 5-FU in human colon cancer DLD-1 cells. Cancer Lett, 2011. 300(2): p. 197-204.

261. Valentine, J.M., S. Kumar, and A. Moumen, A p53-independent role for the MDM2 antagonist Nutlin-3 in DNA damage response initiation. BMC Cancer, 2011. 11: p. 79.

262. Sur, S., et al., A panel of isogenic human cancer cells suggests a therapeutic approach for cancers with inactivated p53. Proc Natl Acad Sci U S A, 2009. 106(10): p. 3964-9.

263. Waldman, T., K.W. Kinzler, and B. Vogelstein, p21 is necessary for the p53mediated G1 arrest in human cancer cells. Cancer Res, 1995. 55(22): p. 5187-90. 
264. Yu, J., et al., PUMA mediates the apoptotic response to p53 in colorectal cancer cells. Proc Natl Acad Sci U S A, 2003. 100(4): p. 1931-6.

265. Huh, W.W. and S.X. Skapek, Childhood rhabdomyosarcoma: new insight on biology and treatment. Curr Oncol Rep, 2010. 12(6): p. 402-10.

266. Dasgupta, R. and D.A. Rodeberg, Update on rhabdomyosarcoma. Semin Pediatr Surg, 2012. 21(1): p. 68-78.

267. Sorensen, P.H., et al., PAX3-FKHR and PAX7-FKHR gene fusions are prognostic indicators in alveolar rhabdomyosarcoma: a report from the children's oncology group. J Clin Oncol, 2002. 20(11): p. 2672-9.

268. Olanich, M.E. and F.G. Barr, A call to ARMS: targeting the PAX3-FOXO1 gene in alveolar rhabdomyosarcoma. Expert Opin Ther Targets, 2013. 17(5): p. 60723.

269. Kashi, V.P., M.E. Hatley, and R.L. Galindo, Probing for a deeper understanding of rhabdomyosarcoma: insights from complementary model systems. Nat Rev Cancer, 2015. 15(7): p. 426-39.

270. Mohan, H.M., et al., Molecular pathways: the role of NR4A orphan nuclear receptors in cancer. Clin Cancer Res, 2012. 18(12): p. 3223-8.

271. Kolluri, S.K., et al., Mitogenic effect of orphan receptor TR 3 and its regulation by MEKK1 in lung cancer cells. Mol Cell Biol, 2003. 23(23): p. 8651-67.

272. Misund, K., et al., NR4A2 is regulated by gastrin and influences cellular responses of gastric adenocarcinoma cells. PLoS One, 2013. 8(9): p. e76234.

273. Tenga, A., et al., Regulation of Nuclear Receptor Nur77 by miR-124. PLoS One, 2016. 11(2): p. e0148433.

274. Shi, X.B., et al., Tumor suppressive miR-124 targets androgen receptor and inhibits proliferation of prostate cancer cells. Oncogene, 2013. 32(35): p. 4130-8.

275. Peng, X.H., et al., MiR-124 suppresses tumor growth and metastasis by targeting Foxq1 in nasopharyngeal carcinoma. Mol Cancer, 2014. 13: p. 186.

276. Shi, X.B., et al., miR-124 and Androgen Receptor Signaling Inhibitors Repress Prostate Cancer Growth by Downregulating Androgen Receptor Splice Variants, EZH2, and Src. Cancer Res, 2015. 75(24): p. 5309-17.

277. Wang, P., et al., Methylation-mediated silencing of the miR-124 genes facilitates pancreatic cancer progression and metastasis by targeting Racl. Oncogene, 2014. 33(4): p. 514-24.

278. Zhang, S., et al., Downregulation of miR-132 by promoter methylation contributes to pancreatic cancer development. Carcinogenesis, 2011. 32(8): p. 1183-9.

279. Penna, E., F. Orso, and D. Taverna, miR-214 as a key hub that controls cancer networks: small player, multiple functions. J Invest Dermatol, 2015. 135(4): p. 960-9.

280. Juan, A.H., et al., Mir-214-dependent regulation of the polycomb protein Ezh2 in skeletal muscle and embryonic stem cells. Mol Cell, 2009. 36(1): p. 61-74.

281. Liu, J., et al., MicroRNA-214 promotes myogenic differentiation by facilitating exit from mitosis via down-regulation of proto-oncogene $N$-ras. J Biol Chem, 2010. 285(34): p. 26599-607.

282. Svalina, M.N. and C. Keller, YAPping about differentiation therapy in muscle cancer. Cancer Cell, 2014. 26(2): p. 154-5. 
283. Huang, H.J., et al., MiR-214 and N-ras regulatory loop suppresses rhabdomyosarcoma cell growth and xenograft tumorigenesis. Oncotarget, 2014. 5(8): p. 2161-75.

284. Kim, H.K., et al., Muscle-specific microRNA miR-206 promotes muscle differentiation. J Cell Biol, 2006. 174(5): p. 677-87.

285. Rao, P.K., et al., Myogenic factors that regulate expression of muscle-specific microRNAs. Proc Natl Acad Sci U S A, 2006. 103(23): p. 8721-6.

286. Hinson, A.R., et al., Human rhabdomyosarcoma cell lines for rhabdomyosarcoma research: utility and pitfalls. Front Oncol, 2013. 3: p. 183. 
APPENDIX. SUPPLEMENTAL DATA FOR CHAPTER 3 
Table A-1. List of miRNAs screened.

\begin{tabular}{|c|c|c|c|c|c|}
\hline miRNA ID & mirBase v14.0 Accession \# & Log2 fold change & Std. dev. & $P$-value & Significance \\
\hline hsa-miR-335 & MIMAT0000765 & -0.475 & 0.0223 & $<0.0001$ & $* * * *$ \\
\hline hsa-miR-34c & MIMAT0000686 & -0.475 & 0.1028 & $<0.0001$ & $* * * *$ \\
\hline hsa-miR-144 & MIMAT0000436 & -0.444 & 0.0696 & $<0.0001$ & $* * * *$ \\
\hline hsa-miR-214 & MIMAT0000271 & -0.379 & 0.0942 & 0.0002 & $* * *$ \\
\hline hsa-miR-191 & MIMAT0000440 & -0.371 & 0.1471 & 0.0002 & $* * *$ \\
\hline hsa-miR-15a & MIMAT0000068 & -0.362 & 0.0343 & 0.0004 & $* * *$ \\
\hline hsa-miR-155 & MIMAT0000646 & -0.361 & 0.0926 & 0.0004 & $* * *$ \\
\hline hsa-miR-20a & MIMAT0000075 & -0.355 & 0.0296 & 0.0005 & $* * *$ \\
\hline hsa-miR-25 & MIMAT0000081 & -0.352 & 0.128 & 0.0006 & $* * *$ \\
\hline hsa-miR-122 & MIMAT0000421 & -0.348 & 0.0902 & 0.0007 & $* * *$ \\
\hline hsa-miR-140 & MIMAT0000431 & -0.333 & 0.015 & 0.0016 & ** \\
\hline hsa-miR-17 & MIMAT0000070 & -0.325 & 0.0957 & 0.0024 & $* *$ \\
\hline hsa-let-7b & MIMAT0000063 & -0.314 & 0.0205 & 0.0039 & $* *$ \\
\hline hsa-miR-363 & MIMAT0000707 & -0.303 & 0.1958 & 0.0066 & ** \\
\hline hsa-miR-218 & MIMAT0000275 & -0.297 & 0.0494 & 0.0085 & ** \\
\hline hsa-miR-9 & MIMAT0000441 & -0.297 & 0.0711 & 0.0086 & $* *$ \\
\hline hsa-miR-132 & MIMAT0000426 & -0.297 & 0.1684 & 0.0086 & ** \\
\hline hsa-miR-21 & MIMAT0000076 & -0.274 & 0.0699 & 0.0224 & * \\
\hline hsa-miR-184 & MIMAT0000454 & -0.268 & 0.0717 & 0.0289 & * \\
\hline hsa-miR-32 & MIMAT0000090 & -0.266 & 0.0533 & 0.0317 & * \\
\hline hsa-miR-148a & MIMAT0000243 & -0.251 & 0.1481 & 0.0553 & ns \\
\hline hsa-miR-10b & MIMAT0000254 & -0.237 & 0.0745 & 0.0892 & $\mathrm{~ns}$ \\
\hline hsa-miR-30c & MIMAT0000244 & -0.228 & 0.138 & 0.1213 & ns \\
\hline hsa-miR-210 & MIMAT0000267 & -0.213 & 0.0814 & 0.1895 & ns \\
\hline hsa-miR-128b & MIMAT0031095 & -0.203 & 0.0917 & 0.2513 & ns \\
\hline hsa-miR-92a & MIMAT0000092 & -0.199 & 0.0536 & 0.2774 & ns \\
\hline hsa-miR-133b & MIMAT0000770 & -0.195 & 0.1246 & 0.3096 & ns \\
\hline hsa-miR-143 & MIMAT0000435 & -0.184 & 0.1607 & 0.3967 & ns \\
\hline hsa-miR-150 & MIMAT0000451 & -0.173 & 0.1322 & 0.5083 & ns \\
\hline hsa-miR-29a & MIMAT0000086 & -0.171 & 0.1333 & 0.5269 & ns \\
\hline hsa-miR-222 & MIMAT0000279 & -0.164 & 0.0358 & 0.5981 & ns \\
\hline hsa-miR-98 & MIMAT0000096 & -0.161 & 0.0807 & 0.6303 & ns \\
\hline hsa-miR-18a & MIMAT0000072 & -0.158 & 0.046 & 0.6677 & ns \\
\hline hsa-miR-181b & MIMAT0000257 & -0.143 & 0.1663 & 0.8227 & ns \\
\hline hsa-miR-27a & MIMAT0000084 & -0.139 & 0.1611 & 0.8586 & ns \\
\hline hsa-miR-301a & MIMAT0000688 & -0.132 & 0.1036 & 0.9086 & ns \\
\hline hsa-miR-130a & MIMAT0000425 & -0.127 & 0.0528 & 0.9394 & ns \\
\hline hsa-miR-206 & MIMAT0000462 & -0.126 & 0.1139 & 0.9474 & ns \\
\hline hsa-let-7e & MIMAT0000066 & -0.119 & 0.1312 & 0.9705 & ns \\
\hline hsa-miR-127 & MIMAT0004604 & -0.117 & 0.2329 & 0.9794 & ns \\
\hline hsa-miR-205 & MIMAT0000266 & -0.114 & 0.113 & 0.9807 & ns \\
\hline hsa-miR-23b & MIMAT0000418 & -0.113 & 0.0235 & 0.9812 & ns \\
\hline hsa-miR-19a & MIMAT0000073 & -0.112 & 0.1182 & 0.982 & ns \\
\hline hsa-miR-212 & MIMAT0000269 & -0.106 & 0.1397 & 0.9843 & ns \\
\hline hsa-miR-34a & MIMAT0000255 & -0.089 & 0.2004 & 0.9983 & ns \\
\hline hsa-miR-126 & MIMAT0000445 & -0.078 & 0.1309 & 0.9986 & ns \\
\hline hsa-miR-193b & MIMAT0002819 & -0.072 & 0.1828 & 0.9988 & ns \\
\hline hsa-miR-16 & MIMAT0000069 & -0.067 & 0.0803 & 0.9989 & ns \\
\hline hsa-miR-100 & MIMAT0000098 & -0.059 & 0.0657 & 0.999 & ns \\
\hline hsa-miR-135b & MIMAT0000758 & -0.059 & 0.0515 & 0.999 & ns \\
\hline hsa-miR-193a & MIMAT0004614 & -0.057 & 0.0549 & 0.9991 & ns \\
\hline
\end{tabular}


Table A-1. (Continued).

\begin{tabular}{lccccc}
\hline miRNA ID & mirBase v14.0 Accession \# & Log2 fold change & Std. dev. & $P$-value & Significance \\
\hline hsa-miR-146b & MIMAT0002809 & -0.051 & 0.1313 & 0.9992 & $\mathrm{~ns}$ \\
hsa-miR-125a & MIMAT0000443 & -0.042 & 0.088 & 0.9994 & $\mathrm{~ns}$ \\
hsa-miR-181d & MIMAT0002821 & -0.035 & 0.1467 & 0.9995 & $\mathrm{~ns}$ \\
hsa-miR-7 & MIMAT0000252 & -0.031 & 0.0815 & 0.9996 & $\mathrm{~ns}$ \\
hsa-let-7f & MIMAT0000067 & -0.029 & 0.1111 & 0.9996 & $\mathrm{~ns}$ \\
hsa-miR-96 & MIMAT0000095 & -0.024 & 0.1182 & 0.9997 & $\mathrm{~ns}$ \\
hsa-let-7d & MIMAT0000065 & -0.013 & 0.0577 & 0.9999 & $\mathrm{~ns}$ \\
hsa-miR-373 & MIMAT0000726 & -0.002 & 0.0811 & $>0.9999$ & $\mathrm{~ns}$ \\
hsa-miR-183 & MIMAT0000261 & 0.0092 & 0.1278 & 0.9998 & $\mathrm{~ns}$ \\
hsa-miR-378 & MIMAT0000732 & 0.0094 & 0.0893 & 0.9998 & $\mathrm{~ns}$ \\
hsa-let-7a & MIMAT0000062 & 0.0112 & 0.1743 & 0.9997 & $\mathrm{~ns}$ \\
hsa-miR-203 & MIMAT0000264 & 0.0438 & 0.1135 & 0.9991 & $\mathrm{~ns}$ \\
hsa-let-7c & MIMAT0000064 & 0.0536 & 0.1421 & 0.999 & $\mathrm{~ns}$ \\
hsa-miR-200c & MIMAT0000617 & 0.0546 & 0.1715 & 0.999 & $\mathrm{~ns}$ \\
hsa-miR-29b & MIMAT0000100 & 0.0596 & 0.0889 & 0.9988 & $\mathrm{~ns}$ \\
hsa-miR-10a & MIMAT0000253 & 0.072 & 0.1945 & 0.9985 & $\mathrm{~ns}$ \\
hsa-miR-146a & MIMAT0000449 & 0.0773 & 0.1303 & 0.9983 & $\mathrm{~ns}$ \\
hsa-miR-199a & MIMAT0000232 & 0.1012 & 0.0518 & 0.9824 & $\mathrm{~ns}$ \\
hsa-miR-181c & MIMAT0000258 & 0.167 & 0.1058 & 0.4707 & $\mathrm{~ns}$ \\
hsa-miR-124 & MIMAT0000422 & 0.2023 & 0.0932 & 0.1954 & $\mathrm{~ns}$ \\
hsa-miR-20b & MIMAT0001413 & 0.2176 & 0.0801 & 0.1232 & $\mathrm{~ns}$ \\
hsa-miR-27b & MIMAT0000419 & 0.2827 & 0.1402 & 0.0107 & $*$ \\
hsa-miR-148b & MIMAT0000759 & 0.3025 & 0.0726 & 0.0043 & $* *$ \\
hsa-miR-181a & MIMAT0000256 & 0.3268 & 0.0741 & 0.0014 & $* *$ \\
\hline
\end{tabular}

****,$P \leq 0.0001 ; * * *, P \leq 0.001 ; * *, P \leq 0.01 ; *, P \leq 0.05 ;$ ns,$P>0.05$ 
Figure A-1. Correlation of miR-34a and $N R 4 A 2$ expression in rectum adenocarcinoma patients.

(a) Regression analysis was used to determine the correlation between expression of miR$34 \mathrm{a}$ and NR4A2 in a subset of 97 patient samples from the TGCA rectum adenocarcinoma dataset. (b) TP53 expression was determined using HiSeq data from the 97 patient samples and the distribution of expression is represented. (c) Regression analysis was used to determine the correlation between expression of mir-34a and NR4A2 in a smaller subset of 74 patient samples with higher TP53 expression. 
a.

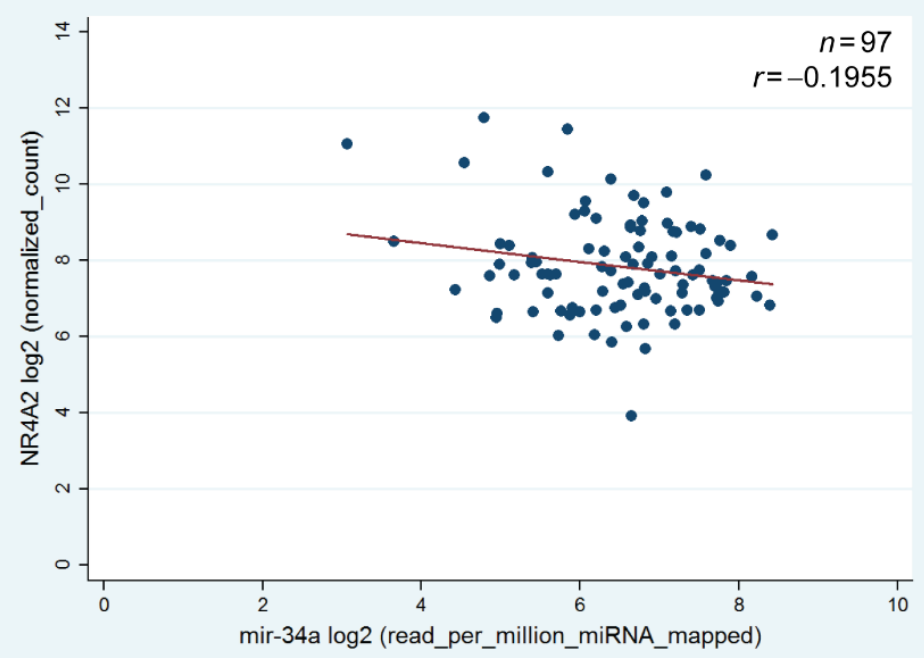

b.

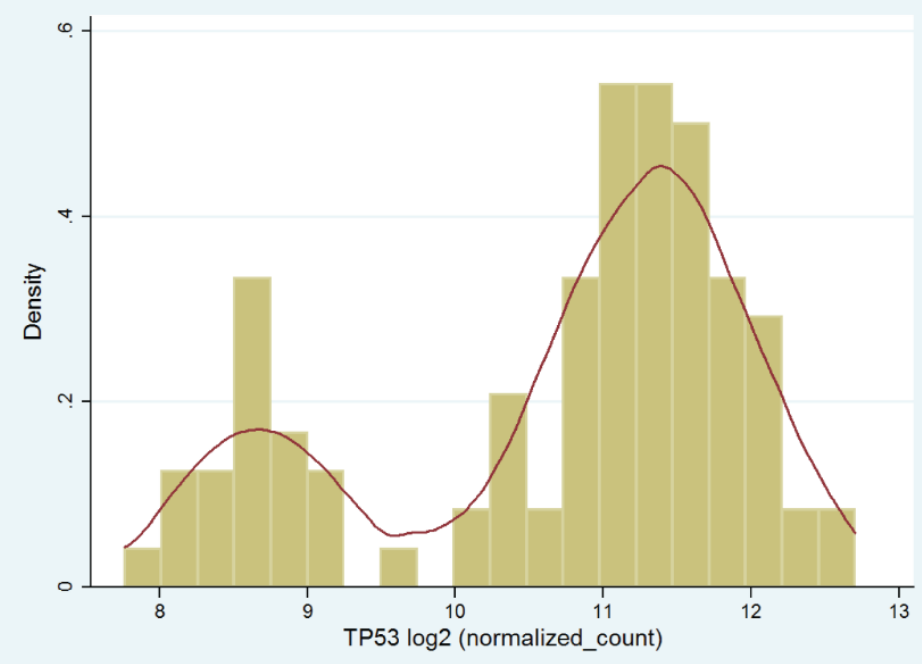

c.

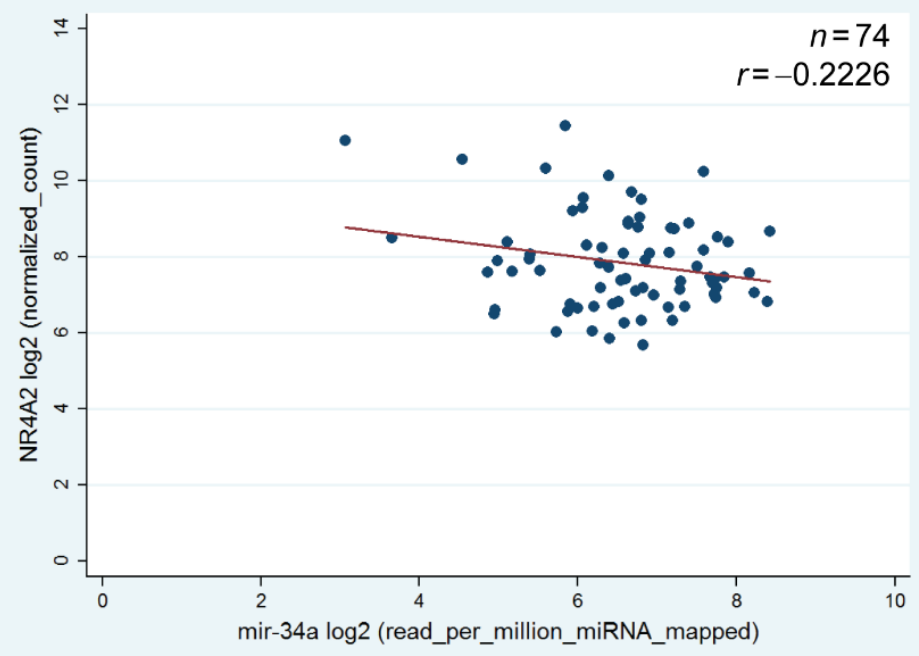


Figure A-2. miR-34a correlation matrix in rectum adenocarcinoma patients. (a) Expression correlations between miR-34a and indicated genes were determined using regression analysis in a subset of 97 patient samples from the TGCA rectum adenocarcinoma dataset. (b) Regression analysis was used to determine the correlation between expression of miR-34a and indicated genes in a smaller subset of 74 patient samples with higher TP53 expression. 
a.

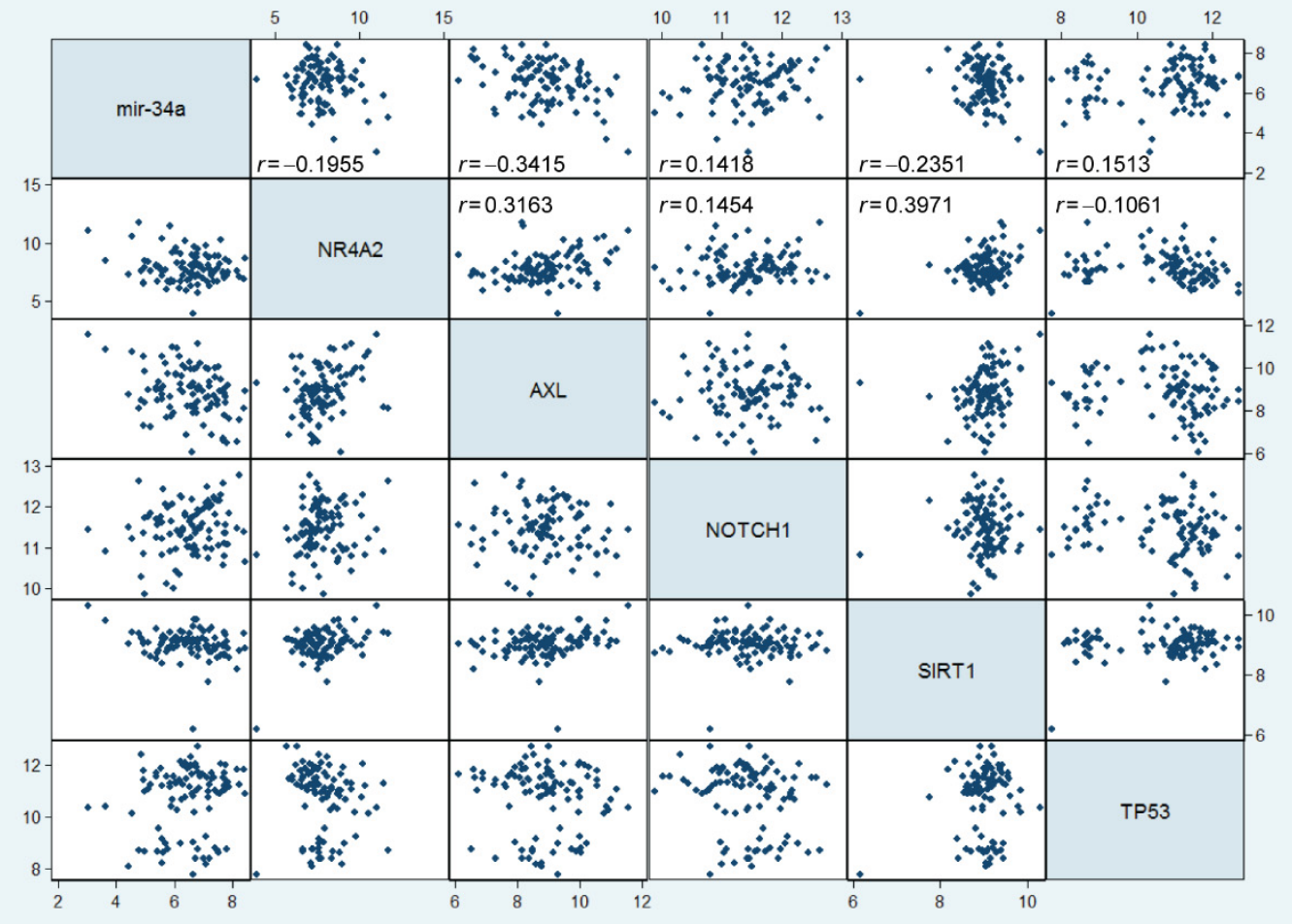

b.

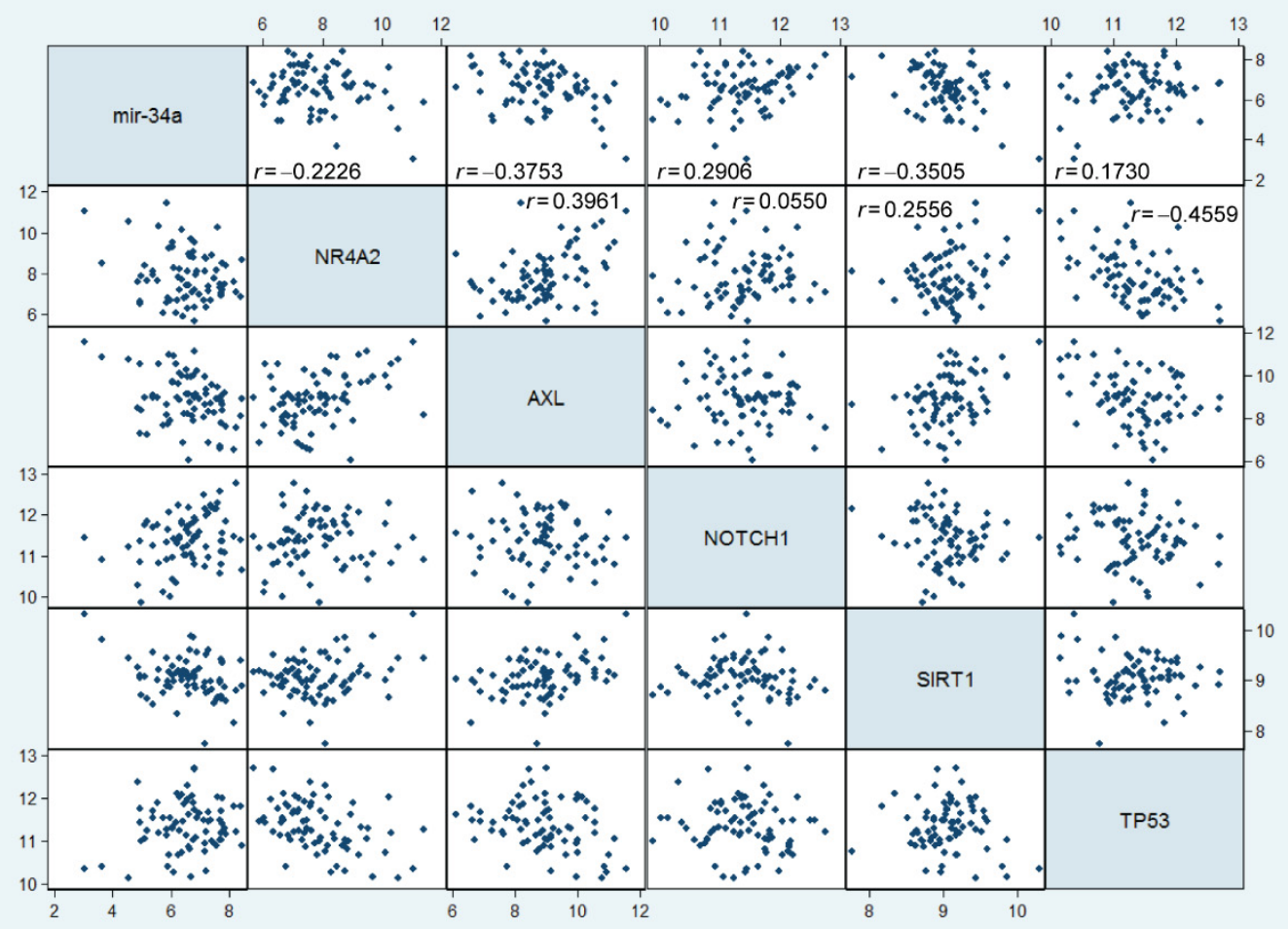


a.

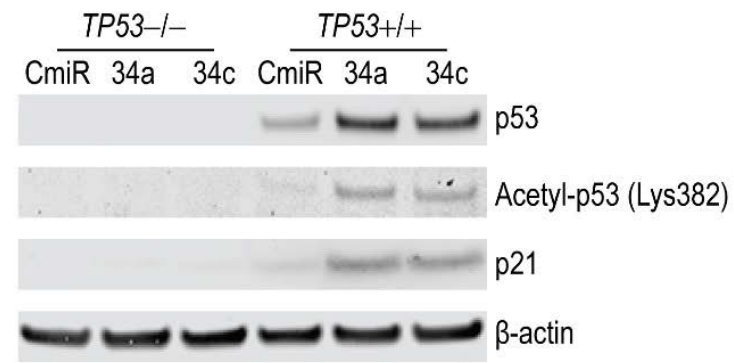

C.

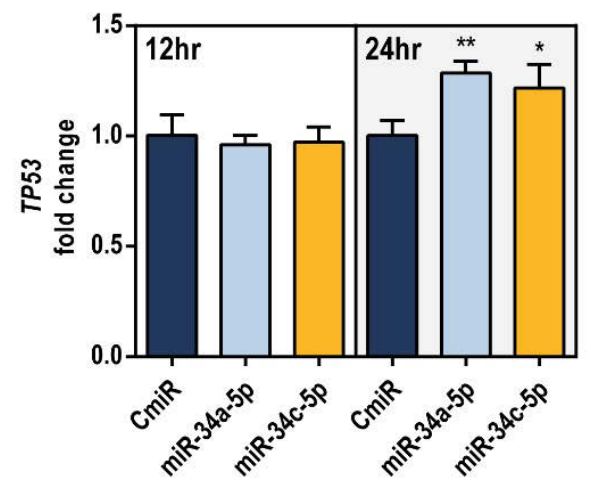

b.

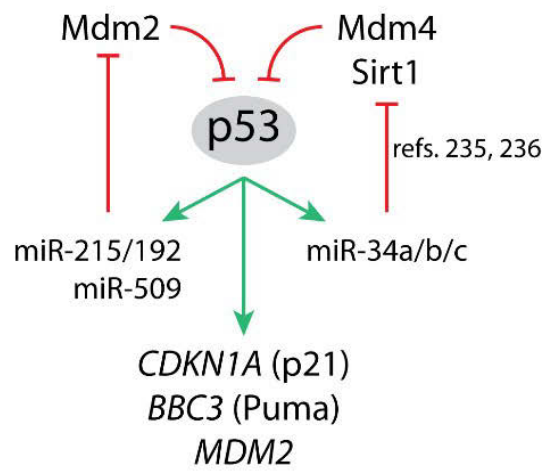

d.

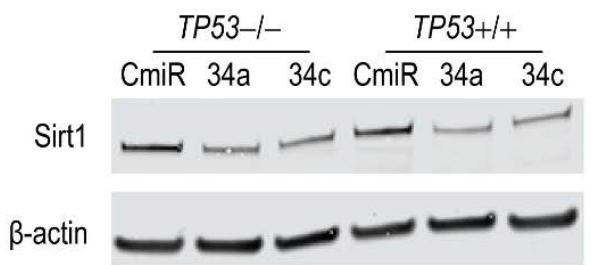

Figure A-3. Overexpression of miR-34 increases p53 protein and acetylation levels.

(a) Control (labeled as CmiR) or miR-34a-5p (34a) or miR-34c-5p (34c) mimics (10 nM) were transfected into HCT116 isogenic cell lines for $48 \mathrm{~h}$, after which SDS-PAGE gel electrophoresis and Western blot analysis of the indicated proteins from whole cell lysates $(45 \mu \mathrm{g})$ was performed. (b) A schematic of the previously described positivefeedback mechanisms involving p53 and its transcriptionally regulated miRNAs. (c) Expression of TP53 was determined using a TaqMan qPCR probe after transfection of control (CmiR), miR-34a-5p, or miR-34c-5p mimics (10 nM) into HCT116 wild-type (WT) cells for the times indicated. Gene expression was normalized to GAPDH. The value for the CmiR-transfected WT cells at each time point was set as 1 . The statistical significance of the results for miR-34 transfections, compared to those for the control, at each time point was calculated using a one-way ANOVA and Dunnett's test for multiple comparisons. ${ }^{* *}, P \leq 0.01 ;{ }^{*}, P \leq 0.05$. (d) Whole-cell lysates $(45 \mu \mathrm{g})$ from HCT116 $6^{\text {TPS3- }}$ (TP53-/-) and HCT116 wild-type (TP53+/+) cells transfected for $48 \mathrm{~h}$ with CmiR, miR$34 a-5 p(34 a)$, or miR-34c-5p (34c) mimics were assessed for expression of Sirt1 and $\beta$ actin protein by performing SDS-PAGE gel electrophoresis and Western blot analysis. 
a.

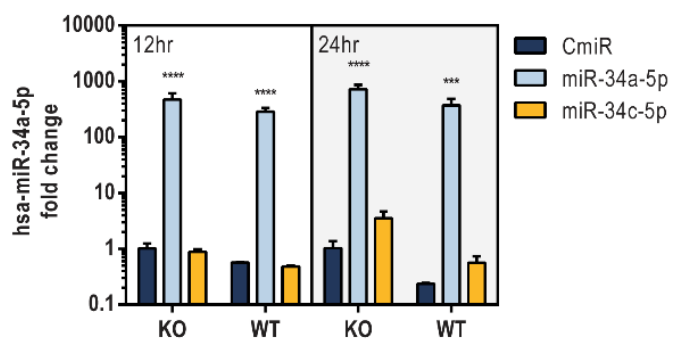

C.

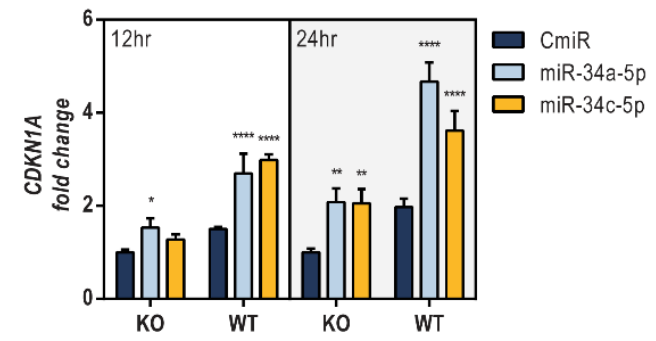

b.

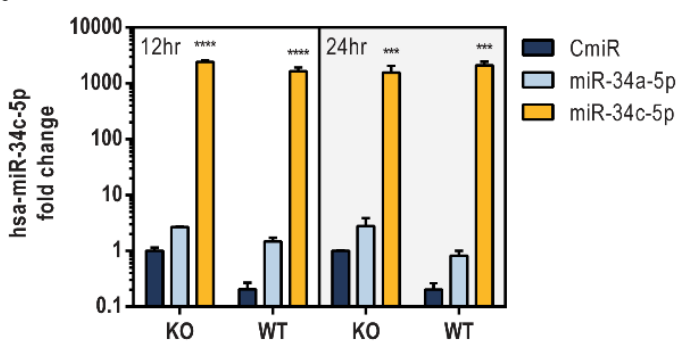

d.

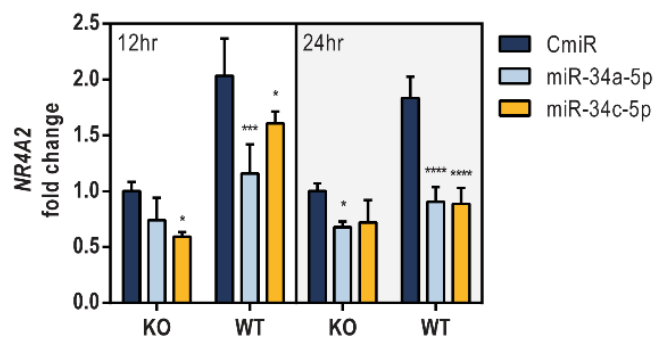

Figure A-4. Overexpression of miR-34 decreases $N R 4 A 2$ in RKO colorectal cancer cells.

Mature miR-34a-5p and miR-34c-5p mimics (10 nM) were transfected into RKO ${ }^{T P 53-/-}$ (KO) or RKO wild-type (WT) cells for 12 or $24 \mathrm{~h}$. Expression of hsa-miR-34a-5p (a), hsa-miR-34c-5p (b), CDKN1A (c), and NR4A2 (d) was determined using TaqMan qPCR probes. Mature miRNA expression was normalized to RNU48, and $C D K N 1 A$ and NR4A2 were normalized to $G A P D H$. The value for the CmiR-transfected $\mathrm{KO}$ cells at each time point was set as 1 . The statistical significance of the results for miR-34 transfections for each cell line, compared to those for the control, at each time point was calculated using a two-way ANOVA and Dunnett's test for multiple comparisons. $* * * *, P \leq 0.0001$; ***, $P \leq 0.001 ; * *, P \leq 0.01 ; *, P \leq 0.05$. 
a.
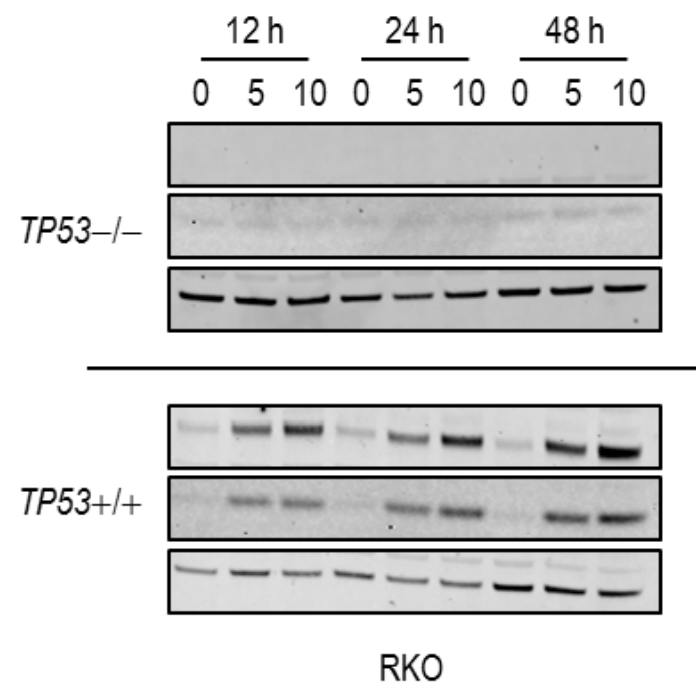

b.

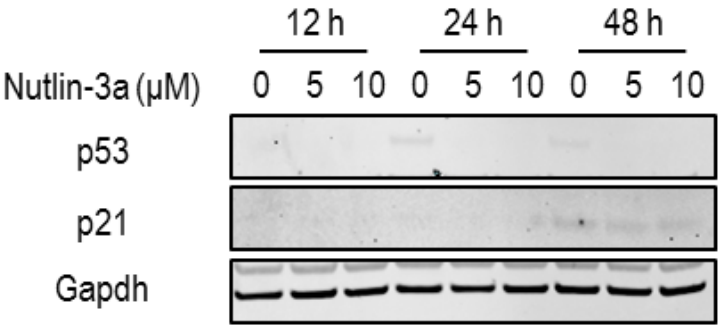

p53

p21

Gapdh

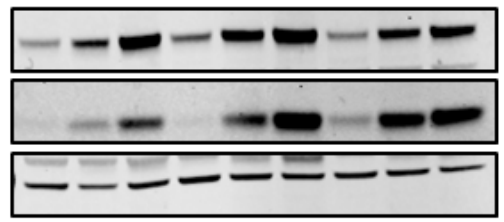

SW48

Figure A-5. Nutlin-3a enhances p53 and p21 protein levels in RKO and SW48 colorectal cancer cell lines.

RKO (a) and SW48 (b) colorectal cancer cell lines that are isogenic for p53 expression (TP53-/-, top; TP53+/+, bottom) were treated for the indicated time periods with vehicle (DMSO) $(0 \mu \mathrm{M})$ or Nutlin-3a (5 or $10 \mu \mathrm{M})$. Whole-cell lysates were assessed for expression of $\mathrm{p} 53$, p21, and Gapdh protein by performing SDS-PAGE gel electrophoresis. 

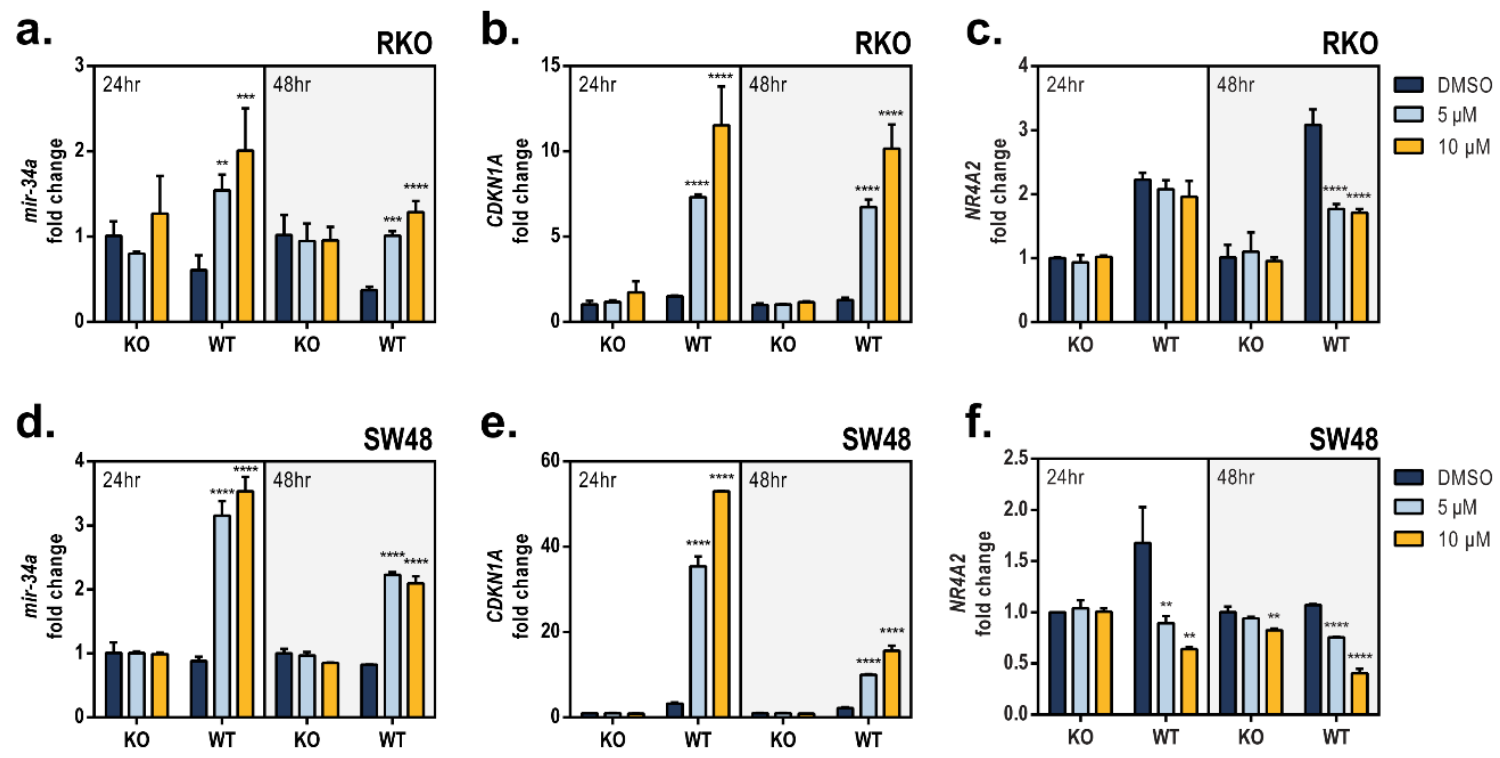

Figure A-6. p53 activation by Nutlin-3a decreases $N R 4 A 2$ in RKO and SW48 cell lines.

$\mathrm{RKO}^{\text {TP53-/- }}$ (KO) and RKO wild-type (WT) (a-c) or SW48 ${ }^{T P 53-/-}(\mathrm{KO})$ and SW48 wildtype (WT) cells (d-f) were treated with vehicle control (DMSO) or Nutlin-3a (5 or 10 $\mu \mathrm{M})$ for 24 or $48 \mathrm{~h}$. Expression of mir-34a (a, d), CDKN1A (b, e), and NR4A2 (c, f) was determined using TaqMan qPCR probes (normalized to GAPDH). The value for the DMSO-treated KO cells at each time point was set as 1 . The statistical significance of the results obtained with Nutlin-3a treatments for each cell line, compared to those obtained with DMSO, for each time point was calculated using a two-way ANOVA and Dunnett's test for multiple comparisons. ***, $P \leq 0.0001 ; * * *, P \leq 0.001 ; * *, P \leq 0.01$. 

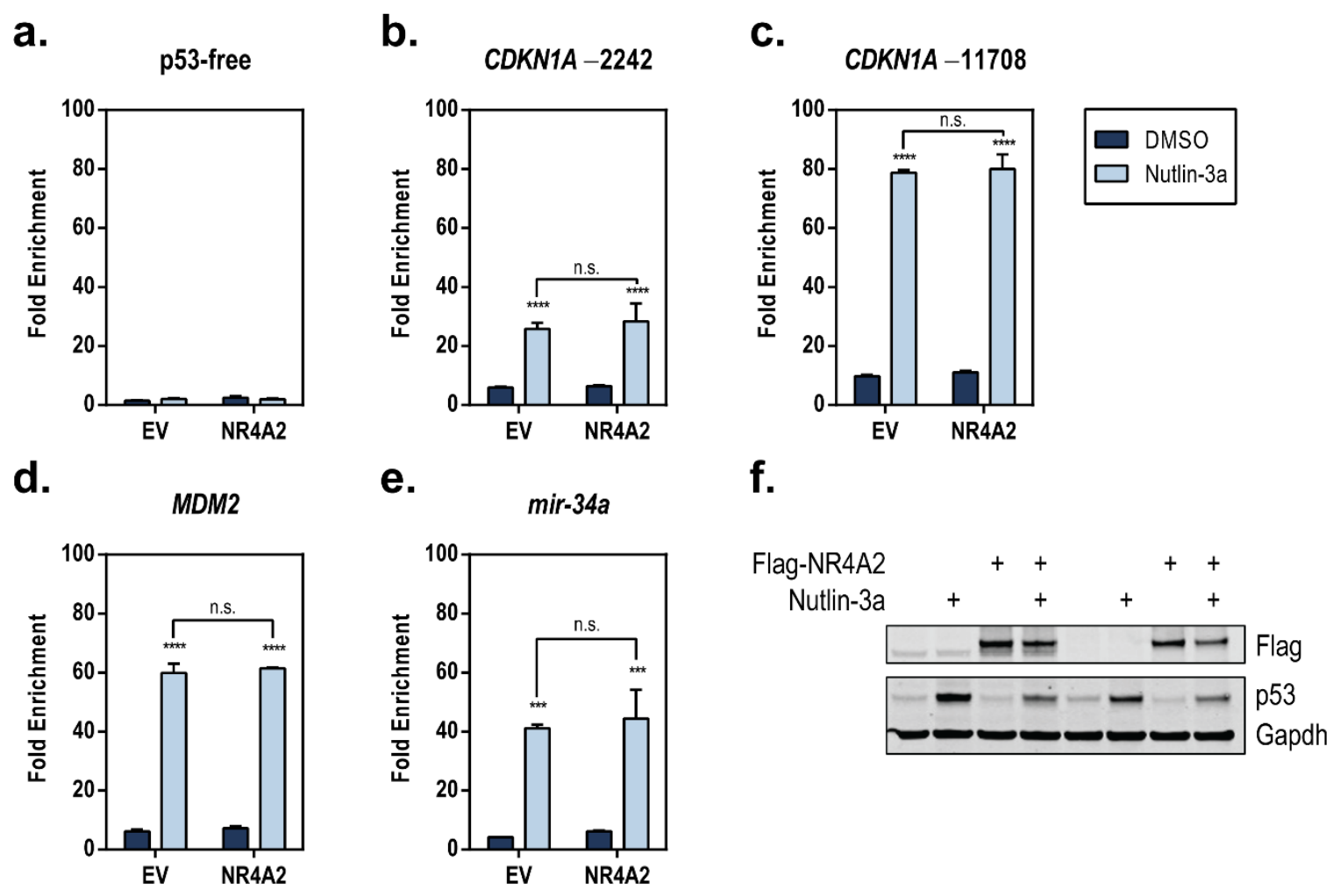

\section{Figure A-7. Overexpression of NR4A2 does not affect binding of p53 to target gene promoters.}

HCT116 wild-type cells were transduced with empty vector (EV) or 3xFlag-NR4A2 (NR4A2) lentivirus. After $16 \mathrm{~h}$, the medium was changed and the cells were grown in culture for a total of $48 \mathrm{~h}$. The cells were then reseeded into $150-\mathrm{mm}^{2}$ flasks and treated for $6 \mathrm{~h}$ with vehicle (DMSO) or Nutlin-3a $(10 \mu \mathrm{M})$. The chromatin immunoprecipitation protocol was performed and the occupancy of p53 at the p53-free (a), CDKN1A-2242 (b), CDKN1A-11708 (c), MDM2 (d), and mir-34a (e) promoter regions is shown (represented as fold-enrichment over IgG). (f) Nuclear-enriched extracts used as input for the ChIP were resolved on a 4-12\% SDS-PAGE gradient gel and probed with antibodies against Flag (indicating NR4A2), p53, and Gapdh. The statistical significance of the results was determined using a two-way ANOVA with Tukey's multiple comparison test. The significance of the differences within each transduction group (****, $P \leq 0.0001$; $* * *, P \leq 0.001)$ and between transduction groups (n.s., $P>0.05)$ is represented. 
Table A-2. List of primers used.

\begin{tabular}{|c|c|c|c|}
\hline Primer name & Sequence (5' to $\left.3^{\prime}\right)$ & Type & Use \\
\hline SpeI-3xFlag fwd & $\begin{array}{l}\text { ATA CTA GTC CAC } \\
\text { CAT GGA CTA CAA } \\
\text { AGA CC }\end{array}$ & Cloning & $\begin{array}{l}\text { Subcloned 3xFlag- } \\
\text { NR4A2 into pSIN } \\
\text { lenti expression } \\
\text { plasmid }\end{array}$ \\
\hline BamHI-Nurr1 rev & $\begin{array}{l}\text { ATG GAT CCC TAG } \\
\text { AAA GGT AAA GTG } \\
\text { TCC A }\end{array}$ & Cloning & $\begin{array}{l}\text { Subcloned 3xFlag- } \\
\text { NR4A2 into pSIN } \\
\text { lenti expression } \\
\text { plasmid }\end{array}$ \\
\hline EF1a fwd seq & $\begin{array}{l}\text { TCA AGC CTC AGA } \\
\text { CAG TGG TTC }\end{array}$ & Sequencing & $\begin{array}{l}\text { Confirm cloning of } \\
\text { 3xFlag-NR4A2 into } \\
\text { pSIN plasmid }\end{array}$ \\
\hline pSIN rev seq & $\begin{array}{l}\text { CCC TAG ATG CAT } \\
\text { GCG GAT CCT TCG }\end{array}$ & Sequencing & $\begin{array}{l}\text { Confirm cloning of } \\
3 x \text { Flag-NR4A2 into } \\
\text { pSIN plasmid }\end{array}$ \\
\hline pEZX-MT01 fwd & $\begin{array}{l}\text { GAT CCG CGA GAT } \\
\text { CCT GAT }\end{array}$ & Sequencing & $\begin{array}{l}\text { Confirm seed region } \\
\text { mutation }\end{array}$ \\
\hline pEZX-MT01 rev & $\begin{array}{l}\text { TTG GCG TTA CTA } \\
\text { TGG GAA CAT }\end{array}$ & Sequencing & $\begin{array}{l}\text { Confirm seed region } \\
\text { mutation }\end{array}$ \\
\hline CDKN1A -3969 bp & ACT ATA TGC TCA & ChIP & p53 ChIP of p53-free \\
\hline RE fwd (p53-free) & $\begin{array}{l}\text { GCC ATT GTG TCT } \\
\text { GCT }\end{array}$ & qPCR & $\begin{array}{l}\text { region in } C D K N 1 A \\
\text { promoter }^{247}\end{array}$ \\
\hline CDKN1A -3969 bp & CCC TCA GCA TCA & ChIP & p53 ChIP of p53-free \\
\hline RE rev (p53-free) & GTG TTA CCA ACC & qPCR & $\begin{array}{l}\text { region in CDKN1A } \\
\text { promoter }^{247}\end{array}$ \\
\hline $\begin{array}{l}\text { CDKN1A -2242 bp } \\
\text { RE fwd }\end{array}$ & $\begin{array}{l}\text { CTG TGG CTC TGA } \\
\text { TTG GCT TT }\end{array}$ & $\begin{array}{l}\text { ChIP } \\
\text { qPCR }\end{array}$ & $\begin{array}{l}\text { p53 ChIP of } C D K N 1 A \\
\text { promoter }^{247}\end{array}$ \\
\hline $\begin{array}{l}\text { CDKN1A -2242 bp } \\
\text { RE rev }\end{array}$ & $\begin{array}{l}\text { CCC TTC CTC ACC } \\
\text { TGA AAA CA }\end{array}$ & $\begin{array}{l}\text { ChIP } \\
\text { qPCR }\end{array}$ & $\begin{array}{l}\text { p53 ChIP of CDKN1A } \\
\text { promoter }^{247}\end{array}$ \\
\hline $\begin{array}{l}\text { CDKN1A }-11708 \\
\text { bp RE fwd }\end{array}$ & $\begin{array}{l}\text { GAG TGG GTG GCT } \\
\text { CAC TCT TC }\end{array}$ & $\begin{array}{l}\text { ChIP } \\
\text { qPCR }\end{array}$ & $\begin{array}{l}\text { p53 ChIP of } C D K N 1 A \\
\text { promoter }^{247}\end{array}$ \\
\hline $\begin{array}{l}\text { CDKN1A }-11708 \\
\text { bp RE rev }\end{array}$ & $\begin{array}{l}\text { CTC GCA TCA GCA } \\
\text { ACT CTG G }\end{array}$ & $\begin{array}{l}\text { ChIP } \\
\text { qPCR }\end{array}$ & $\begin{array}{l}\text { p53 ChIP of CDKNIA } \\
\text { promoter }^{247}\end{array}$ \\
\hline MDM2 p53 RE fwd & $\begin{array}{l}\text { GAT TGG GCC GGT } \\
\text { TCA GTG G }\end{array}$ & $\begin{array}{l}\text { ChIP } \\
\text { qPCR }\end{array}$ & $\begin{array}{l}\text { p53 ChIP of MDM2 } \\
\text { promoter }^{248}\end{array}$ \\
\hline MDM2 p53 RE rev & $\begin{array}{l}\text { CAC AGC TGG GAA } \\
\text { AAT GCA TGG }\end{array}$ & $\begin{array}{l}\text { ChIP } \\
\text { qPCR }\end{array}$ & $\begin{array}{l}\text { p53 ChIP of MDM2 } \\
\text { promoter }^{248}\end{array}$ \\
\hline mir-34a p53 RE fwd & $\begin{array}{l}\text { ACG CTT GTG TTT } \\
\text { CTC AGT CCG }\end{array}$ & $\begin{array}{l}\text { ChIP } \\
\text { qPCR }\end{array}$ & $\begin{array}{l}\text { p53 ChIP of mir-34a } \\
\text { promoter }^{71}\end{array}$ \\
\hline mir-34a p53 RE rev & $\begin{array}{l}\text { TGG TCT AGT TCC } \\
\text { CGC CTC CT }\end{array}$ & $\begin{array}{l}\text { ChIP } \\
\text { qPCR }\end{array}$ & $\begin{array}{l}\text { p53 ChIP of mir-34a } \\
\text { promoter }^{71}\end{array}$ \\
\hline
\end{tabular}




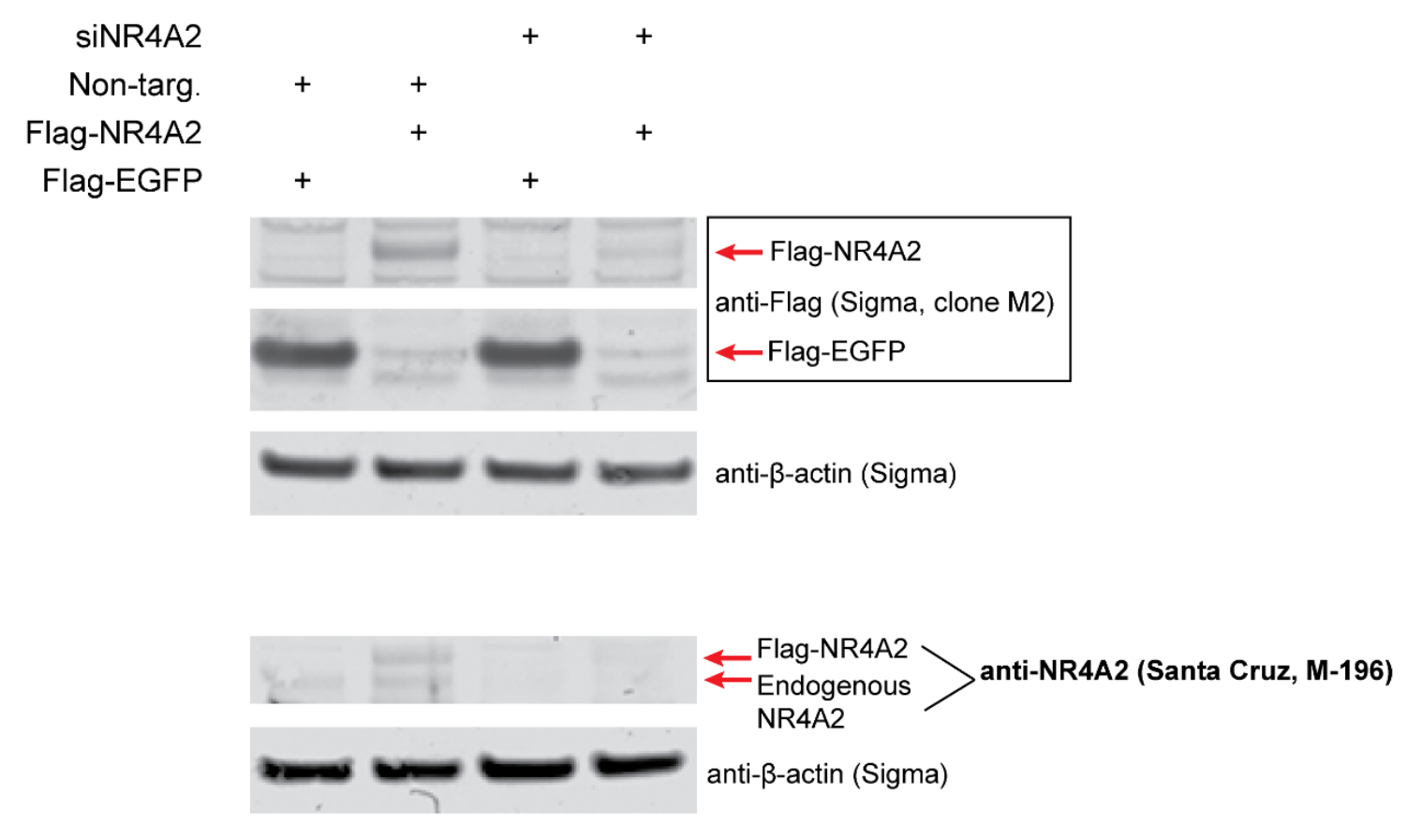

Figure A-8. Validation of an anti-NR4A2 antibody.

HCT116 $6^{\text {TPS3-/- }}$ cells were transfected with nontargeting siRNA (Non-Targ.) or siNR4A2 $(20 \mathrm{nM})$ in $60 \mathrm{~mm}^{2}$ dishes. After $24 \mathrm{~h}$ of siRNA transfection, 3xFlag-EGFP (Flag-EGFP) or 3xFlag-NR4A2 (Flag-NR4A2) were transfected for an additional $48 \mathrm{~h}$. Whole cell lysates $(45 \mu \mathrm{g})$ were resolved on a $4-12 \%$ SDS-PAGE gradient gel and transferred to PVDF membrane using wet transfer (100V constant for $1 \mathrm{~h})$. Membranes were probed with antibodies against Flag (detecting overexpressed NR4A2 or EGFP), NR4A2 (detecting both endogenous NR4A2 and Flag-NR4A2), and $\beta$-actin. The levels of endogenous NR4A2 (detected using anti-NR4A2) and Flag-NR4A2 (detected using either anti-NR4A2 or anti-Flag) decreased in response to siNR4A2 but not Non-Targ. 


\section{VITA}

Jordan A. Beard was born in 1986 in Munford, TN. He graduated in 2008 with a Bachelor of Science in Biology from Samford University. In August of 2009, he joined the Integrated Biomedical Sciences Program at the University of Tennessee Health Science Center. In April 2010, he joined the laboratory of Dr. Taosheng Chen at St. Jude Children's Research Hospital to study the function and regulation of orphan nuclear receptors in cancer. He graduated in May 2016 with the degree of Doctor of Philosophy. He will pursue a postdoctoral fellowship in the laboratory of Dr. William E. Evans at St. Jude Children's Research Hospital. 